\title{
Text Classification with Noisy Class Labels
}

\author{
By \\ Andrea Pagotto \\ A thesis proposal submitted to \\ the Faculty of Graduate and Postdoctoral Affairs \\ in partial fulfilment of \\ the requirements for the degree of \\ Master of Computer Science \\ Ottawa-Carleton Institute for Computer Science \\ School of Computer Science \\ Carleton University \\ Ottawa, Ontario \\ June 2020 \\ (C) Copyright \\ 2020, Andrea Pagotto
}


The undersigned hereby recommend to the Faculty of Graduate and Postdoctoral Affairs acceptance of the thesis,

\section{Text Classification}

with Noisy Class Labels

submitted by

Andrea Pagotto

Dr. Michel Barbeau

(Director, School of Computer Science)

Dr. B. John Oommen

(Thesis Supervisor)

\section{Carleton University}

June 2020 


\begin{abstract}
Text classification is a sub-field of Natural Language Processing (NLP), that involves mapping an input text document to an output topic or label. There are numerous practical applications for text classification, including email spam detection, identifying sentiments in social media, news topic detection, and many more. Due to the importance of the field, there has been much related research in recent years.

Our work in this thesis specifically focuses on the problem of text classification in the setting of a Random Environment. The Random Environment in this application would be noise in the labels of the training data. Label noise is an important issue in classification, with many potential negative consequences, such as decreasing the accuracy of the predictions, and increasing the complexity of the trained models. Designing learning algorithms that help maximize a desired performance measure in such noisy settings, is very valuable for achieving success on real world data.

This thesis also investigates a recently proposed classification method that involves the use of Learning Automata (LA) for text classification. The Tsetlin Automaton (TA) is a simple LA and a versatile learning mechanism. Collections of TAs have recently been harnessed by a learning algorithm called the Tsetlin Machine, to solve complex pattern recognition problems, by learning propositional formulae. The Tsetlin Machine can learn human readable rules made up of clauses using terms from the text. There is currently only a single paper which deals with the application of the Tsetlin Machine to text classification problems, and this thesis builds on the work of that paper. Our research is a comprehensive experimental analysis of the Tsetlin Machine, and of its parameters and its performance, relative to many other classifiers.

Our experiments have shown that classical methods such as Logistic Regression, and the Tsetlin Machine have reasonably low impact on their performance from the injected label noise, while recent state-of-the-art methods in text classification are not quite as robust to certain types of label noise.
\end{abstract}




\section{ACKNOWLEDGEMENTS}

First, I would like to express my sincere gratitude to my supervisor, Professor B. John Oommen, for going above and beyond the expectations of a professor in many ways. I am so grateful to have a professor who cares so deeply about the well-being of his students. Professor Oommen has taken on the role of a fatherly figure, providing me with strong support in all aspects of life. On the academic side, Professor Oommen's expertise in the field of Learning Automata and Machine Learning have been vital for the completion of this research, and his passion for this field of research has been very inspiring. I would also like to thank him for taking much time out of his busy schedule, to go through this thesis page by page with me. His kindness and dedication to helping others reminds me of my own father, and I think my father would be very grateful to know that I have had someone like this looking out for me throughout this journey.

I would also like to offer a very heart-felt thanks to my family, to whom I owe a great deal. I would first like to thank my mother for being there for me through all the ups and downs of this journey, and for trying her best to help in whatever way she could, despite the challenges we both have faced. I would also like to thank my Nonna, who is an amazing inspiration in my life, and visiting her regularly on my way home from Carleton, has not only provided me with much delicious home cooking, but also the moral support that I have needed to keep going. And finally, I would like to thank my father, my main role model in life, who passed away before the completion of these studies. He has been in my mind motivating me every step of the way.

Finally, I would like to thank Carleton's School of Computer Science, including the numerous faculty members who have provided me guidance throughout my studies, and all the friends I made along the way. 


\section{Contents}

1 Introduction 1

1.1 Introduction . . . . . . . . . . . . . . . . . . . . 1

1.2 Objectives and Contributions of the Thesis . . . . . . . . . . . 3

1.3 Contributions ........................ 4

1.4 Motivation of the Thesis . . . . . . . . . . . . . . . 4

1.5 Hardware and Software Used for Simulations . . . . . . . . . . . . . . 6

1.6 Organization of the Thesis . . . . . . . . . . . . . 6

2 Background Survey $\quad 8$

2.1 Introduction . . . . . . . . . . . . . . . . . . . . 8

2.2 Text Representation for Classification . . . . . . . . . . . . . . . 9

2.2.1 Text Pre-processing and Tokenization . . . . . . . . . . . . . . 10

2.2.2 Word Embeddings . . . . . . . . . . . . . . . . . . . . . 12

2.2 .3 Document Vectors . . . . . . . . . . . . . . . . . 16

2.2.4 Summary . . . . . . . . . . . . . . . . . . . . 20

2.3 Supervised Classification . . . . . . . . . . . . . . . . . 20

2.3.1 Text Classification Problem Description . . . . . . . . . . . . 20

2.3.2 Text Classification Methods . . . . . . . . . . . . . . . 21

2.4 Learning Automata (LA) . . . . . . . . . . . . . . . . . . . . . . 29

2.4.1 Overview of LA . . . . . . . . . . . . . . . . . . . . . . . . 29

2.4.2 Types of Learning Automata . . . . . . . . . . . . . . . . . 32

2.4.3 Automata for Text Classification . . . . . . . . . . . . . . 35 
2.5 Classification with Noisy Class Labels . . . . . . . . . . . . . . 37

2.5.1 Overview of Noise in Labels . . . . . . . . . . . . . . . . . . 37

2.5.2 Methods to Handle Noisy Labels in Classification . . . . . . . 43

2.5.3 Experimental Considerations . . . . . . . . . . . 48

2.6 Conclusion . . . . . . . . . . . . . . . . . . . . 49

3 Classification with Injected Label Noise $\quad 51$

3.1 Introduction . . . . . . . . . . . . . . . . . 51

3.2 Classification Experiment Setup . . . . . . . . . . . . 53

3.2.1 Text Classification Data Set . . . . . . . . . . . . 53

3.2.2 Generating Text Vector Representations . . . . . . . . . . 54

3.2 .3 Noise Injection . . . . . . . . . . . . . . . . . . 54

3.2.4 Training Classifiers ................ 55

3.3 Noise Injection Methods . . . . . . . . . . . . . . . . 56

3.3.1 Noise Types . . . . . . . . . . . . . . . . 56

3.3.2 Analysis of Noise Detection . . . . . . . . . . . . 62

3.4 Analysis of Results . . . . . . . . . . . . . . . . . 65

3.4.1 Effects of Document Representations . . . . . . . . . . 66

3.4.2 Analysis of Results by Classifier Type . . . . . . . . . . . . 69

3.4.3 Analysis of Border Noise . . . . . . . . . . . . 76

3.5 Conclusion . . . . . . . . . . . . . . . . . 77

4 Learning Automata Classification Approach $\quad 79$

4.1 The Tsetlin Machine Architecture . . . . . . . . . . . . . . . 80

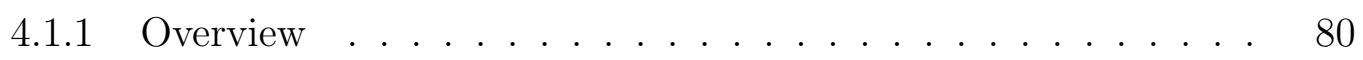

4.1 .2 Architecture . . . . . . . . . . . . . . . 81

4.1.3 Algorithm for Generating Predictions . . . . . . . . . . . . 82

4.1.4 The Training Algorithm . . . . . . . . . . . . . . 84

4.2 Implementation Details . . . . . . . . . . . . . . . 87

4.2.1 Implementation Overview . . . . . . . . . . . . 87

4.3 XOR Simulation Experiments . . . . . . . . . . . . . 89

4.3.1 XOR Function . . . . . . . . . . . . . . . . . . . 90 
4.3 .2 Results . . . . . . . . . . . . . . . . . . . . . . . 9 91

4.4 IMDb Experiments . . . . . . . . . . . . . . . . . . . . 93

4.4 .1 Effect of Number of Clauses _. . . . . . . . . . . . 96

4.4 .2 Effect of Number of States . . . . . . . . . . . . . 96

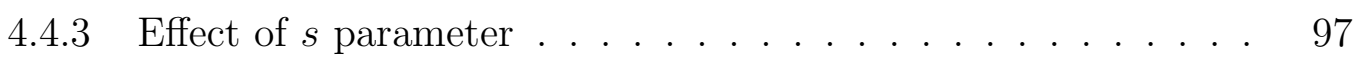

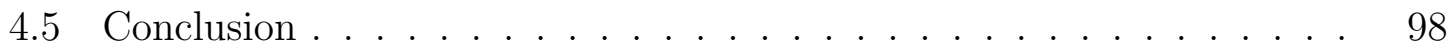

5 Conclusion and Future Work 101

5.1 Summary and Conclusions . . . . . . . . . . . . . . . . . . 101

5.1.1 How do Classification Methods Compare? . . . . . . . . . . 102

5.1.2 What Impacts do Different Types of Label Noise have? . . . . 102

5.1.3 How can Learning Automata be used for Classification with Noisy Labels? . . . . . . . . . . . . . . . . . . . . 103

5.2 Future Work . . . . . . . . . . . . . . . . . . . . . . . . 103

5.2.1 Modifying the Base Automata in the Tsetlin Machine . . . . . 104

5.2.2 Learning Automata Approaches for Text Classification . . . . 106

5.2.3 Characterization of Types of Noise in Text Datasets . . . . . . 106

$\begin{array}{ll}\text { Bibliography } & 107\end{array}$

$\begin{array}{ll}\text { Appendices } & 121\end{array}$

$\begin{array}{ll}\text { A Appendix A } & 122\end{array}$ 


\section{List of Figures}

2.1 NLP pipeline for training a classifier $\ldots \ldots \ldots \ldots$

2.2 GloVe Relationship Representation [84] . . . . . . . . . . . . . . . 14

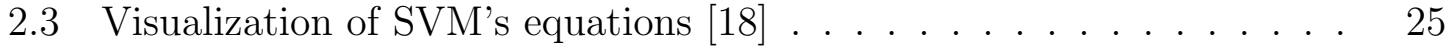

2.4 Overview of general LA learning model . . . . . . . . . . . . 30

2.5 Transition map of the Tsetlin Automata on receiving a reward . . . . 33

2.6 Transition map of the Tsetlin Automata on receiving a penalty . . . . 33

3.1 Overview of experiment methodology . . . . . . . . . . 53

3.2 Scikit-learn classifier training procedure . . . . . . . . . . 55

3.3 Identification of noisy labels with confident learning . . . . . . . 63

3.4 Cleanlab performance in classification with border noise . . . . . . . 64

3.5 Cleanlab performance in classification with random noise . . . . . 65

3.6 Performance of Logistic Regression classifier with 30\% noise . . . . 69

3.7 Effects of parameter tuning on Logistic Regression performance . . . 71

3.8 Performance of each classifier with $30 \%$ random and class dependent

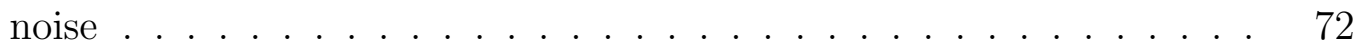

3.9 Performance of each classifier with $30 \%$ border noise . . . . . . . 73

3.10 Training of BERT classifier with different noise types $\ldots \ldots \ldots 75$

3.11 Training of the Tsetlin classifier with different noise types . . . . . 76

4.1 High level architecture of the Tsetlin Machine [39] . . . . . . . . . 82

4.2 Internal architecture of the Tsetlin Machine [39] . . . . . . . . 83 
4.3 Example of a TA chunk implementation with 24 TA [39] . . . . . . 89

4.4 Accuracy vs epoch during training for different parameter settings for the XOR function simulated dataset . . . . . . . . . . . . . . 91

4.5 Accuracy vs epoch during training for different parameter settings for the XOR function simulated dataset . . . . . . . . . . . . . 92

4.6 Effect of parameters s and number of clauses with the XOR dataset . 93

4.7 Effect of parameters s and number of states with the XOR dataset . . 94

4.8 Accuracy vs epoch during training . . . . . . . . . . . . . . 97

4.9 Accuracy vs epoch during training for the IMDb dataset . . . . . . . 98

4.10 Accuracy vs epoch during training for the IMDb dataset . . . . . . . 99 


\section{1 \\ Introduction}

\section{$1.1 \quad$ Introduction}

In recent years, there has been a rapid growth in the amount of unstructured text data available online, including sources such as emails, websites, social media, scientific articles, news articles, and much more. Text can be a rich source of information, but extracting insights from it can be very time-consuming without an automated approach. Research in the field of Natural Language Processing (NLP) has developed over the years to address this task of gaining insight from unstructured data. One fundamental topic within this field of research is text classification, which is the process of assigning categories to unstructured text data.

Text classification is often at the heart of a variety of systems that process large amounts of text data for a broad range of applications. Some examples of applications includes, detecting sentiments from social media data, categorizing news articles 
by topic, identifying toxic messages in online chat, and categorizing emails as spam email. The need for automated text classification for this wide range of applications has driven researchers to develop numerous approaches to address this task, from hand-coded rule-based approaches, to complex Machine Learning (ML) systems. ML is a subset of the field of Artificial Intelligence (AI), which constitutes the study of algorithms that can learn from data, and that do not need to be explicitly programmed. ML algorithms have recently risen to prominence, due to the rapidly increasing amounts of data available.

There are at least three classic paradigms of ML algorithms, that differ based on the type of feedback available to the learning system. These three paradigms, which we allude to, are supervised learning, unsupervised learning, and reinforcement learning. In supervised learning, the algorithm is presented with examples of data to be classified, and the corresponding desired outputs. The inputs in the case of text classification would be the text data, and the outputs would be a category that the text was labelled with, often called the training labels. The objective of the supervised learning algorithm is to learn how to map the inputs to the outputs, and this can be done in a wide variety of ways. This is the most common paradigm used for text classification. Unsupervised learning involves learning to identify categories in data, without being provided with associated labels. Unsupervised learning algorithms are able to uncover structure within the data. This can be used for the purpose of analysis, or as a way to learn features from the structure of the data. A commonly-used unsupervised method for text classification is called topic modelling, which can identify topics in a group of text documents. Informally speaking, reinforcement learning involves software agents taking actions in an environment in order to maximize the notion of cumulative reward. This paradigm has not been, typically, utilized in text classification. However, there is a sub-field of reinforcement learning, known as Learning Automata (LA), that has recently been applied to text classification. This will be the arena in which we work.

In the case of both supervised learning and reinforcement learning paradigms, the algorithms rely on examples provided by humans to be able to learn, by using the labels provided for the text data. Thus, the quality of human judgements set the 
upper bar for the performance of these methods. Humans, however, are inherently prone to error, and so errors can exist in the labels due to mistakes, biases, and inconsistencies in the labelling schemes. This research challenge will be investigated in this thesis, through noise simulation experiments.

Overall, this thesis aims to contribute to the field of text classification with the perspective to see how LA can be applied to this domain, and with a particular focus on testing in the presence of label noise. This thesis also provides a survey of the field of text classification and classification with noisy labels, and provides insights from systematic experiments comparing the performance of a wide array of text classification methods in the presence of various types of label noise.

When one has to test an algorithm, especially in ML applications, one of the hardest things to obtain is data. Indeed, when we speak about testing with noisy labelled samples, it implies that we have access to data sets in which the samples are mislabeled. Typically, as mentioned above, this mislabeling is done due to human error. Alternate noisy data is very hard to obtain. The other option is to artificially generate such data, and this is the strategy that we have chosen.

The way that we have approached this problem is by first obtaining "clean" data. In other words, this means that we assume that we have access to data where the class labels are accurate. We have then taken the data points, item by item, and changed the associated class with a certain probability. The latter probability is a tuning parameter which controls the level of noise, and the programs have been written in a fairly general way so that the noise level can be tuned. Observe that once the artificially garbled data is available, one can use it to train and test any of the existing algorithms, and in particular, use it for the available machine learning techniques and also for the LA schemes that we are working with.

\subsection{Objectives and Contributions of the Thesis}

The main objective of this thesis is to study the use of LA for text classification, and to compare the performance of this novel approach with various other types of classifiers in a Random Environment. In this context, the Random Environment 
constitutes the simulated noise in the training labels for text classification. This, in turn, will allow us to systematically study the effects of label noise on various types of text classification methods. The specific application studied is text classification for sentiment analysis, using the IMDb movie reviews dataset [66]. This application was chosen due to the wide-spread use of this dataset as a benchmark for evaluating text classification methods. This overall objective can be broken down further into two specific objectives, listed as follows:

- We would like to analyze how traditional, state-of-the-art, and the novel LA classification methods compare, and to also analyze the effects of different types of label noise on these classifiers' relative performance with systematic simulation experiments.

- We intend to study the performance of a recently-proposed LA classification method for this application, including investigating and analyzing the classifier's architecture and the effects of its parameters.

\subsection{Contributions}

The contributions of the thesis are the study of the LA approach for text classification, as well as the description of novel types of comparisons of recently-developed text classification methods in the presence of various types of label noise. The primary contribution from this analysis is the determination that the Tsetlin machine is a viable approach for text classification in the presence of label noise.

\subsection{Motivation of the Thesis}

The two main objectives of this thesis are the analysis of the use of LA for text classification, and the comparison of various text classification methods in the presence of label noise. The motivations behind both of these objectives will be described presently. 
This thesis aims to study a novel LA-based approach for text classification. The Tsetlin Automaton (TA) is a simple, versatile learning mechanism that can learn an optimal action in a Random Environment, and uses only a single integer of memory. Due to these beneficial properties of the TA, the authors of [39] recently proposed a strategy by which numerous TAs could be "inter-connected". In other words, they showed how they could utilize this base learning unit to learn complex patterns, with an architecture that coordinates numerous TAs into the Tsetlin Machine. It has been shown that the Tsetlin Machine can have a high accuracy, good interpretability, and computational simplicity. These attributes render it to be an interesting scheme for the application of text classification. In the field of text classification, deep learning has provided great improvements in accuracy. However these methods have a lowlevel of interpretability. The Tsetlin Machine, however, enables learning to represent the classes with simple, human readable conjunctive clauses. This application of the Tsetlin Machine to text classification was recently investigated in a study on medical texts, by Berge et al. [9]. This method has shown to be promising, and so it will be further studied in this thesis. Our aim is to further gain understanding into the architecture and performance of this classification method, relative to other methods. As far as we know, there is only a single publication so far which has applied the Tsetlin Machine for the task of text classification, and this contributes to the novelty of our research.

This thesis also provides a systematic study of both traditional and recently developed text classification methods to analyze their relative performance in the presence of label noise. The development of supervised learning algorithms and methods that can handle datasets with class label noise is a problem of great practical importance, as most applications of ML classification on real-world data are noisy. Specifically, in the domain of text classification, alluded to above, labels provided by humans can often be prone to errors. Text annotation tasks can, often, involve very subjective labels, such as sentiments, that are not clearly defined, and even expert annotators can make mistakes on these types of annotation tasks. Testing robust methods for label noise is well studied across many domains in ML, with a particular focus in the domain of computer vision. There are far fewer existing studies with a focus on 
applications in NLP, and in particular, in analyzing the more recent developments in methods used for text classification, and so this thesis aims to contribute to this area.

\subsection{Hardware and Software Used for Simulations}

In this section, we briefly provide specifics about the hardware and software used for the implementations and simulations presented in this thesis.

The hardware used for the thesis was a GPU virtual machine from Carleton University, and a desktop computer. The Carleton computer had a GeForce GTX 1080 Ti, CUDA Driver 10.0, 11 GB memory, 3584 CUDA Cores, and the operating system was Ubuntu 18.04. The desktop computer had a Mac operating system, Version 10.15, and had a $3.1 \mathrm{GHz}$ Quad-Core Intel Core i5 processor, and 16 GB memory.

The primary language used for running simulations was 'Python', specifically Python with version 3.6. The language ' $\mathrm{C}$ ' was used for the implementation of the Tsetlin Machine.

\subsection{Organization of the Thesis}

The organization of the thesis is as follows:

- In Chapter 2, we present a literature review of the relevant fields including the various aspects of text classification, the challenges of noise in the training labels, a survey of the field of LA, and finally how LA can be applied to text classification.

- In Chapter 3, we present the results of extensive experiments to compare methods for text classification. Their relevant results will be explained and discussed. This chapter will compare many text pre-processing methods, various types of classifiers, as well as many different types of noise injected into the training labels. The dataset used for these experiments is the IMDb text classification dataset, for classifying sentiment in movie reviews. 
- In Chapter 4, we investigate in great detail, the LA approach for classification, i.e., the Tsetlin Machine. The architecture will be thoroughly explained and tested. We will test its parameters with various experiments, using a simulated dataset and the IMDb dataset, to characterize the effects of each parameter.

- Finally, Chapter 5 concludes the thesis. It will provide a summary of each of the previous chapters. It will also discuss, in detail, some ideas for future work in this field of research. 


\section{2 \\ Background Survey}

\section{$2.1 \quad$ Introduction}

Text classification problems have been widely studied and applied in many real applications over the last few decades, including spam email detection, sentiment analysis, and text organization, and the retrieval of text from various sources including social media feeds, scientific literature, and new articles.

The following sections will discuss previous works relevant to this thesis on text classification when it is achieved in the presence of noisy labels. The main three topics reviewed will be text representation including how to transform the text into a representation for input into a classifier in Section 2.2, classification using Machine Learning (ML) methods in Section 2.3.2, and classification in the presence of noisy labels in Section 2.5. 


\subsection{Text Representation for Classification}

Most text classification systems consist of several phases: text pre-processing and tokenization, feature extraction and dimension reduction to obtain vector representations of the documents, and finally, classifier selection and training. The final trained classifier can then be used to perform predictions on test data for evaluation. A simple example of a possible training pipeline for processing text for classification is shown in Figure 2.1.

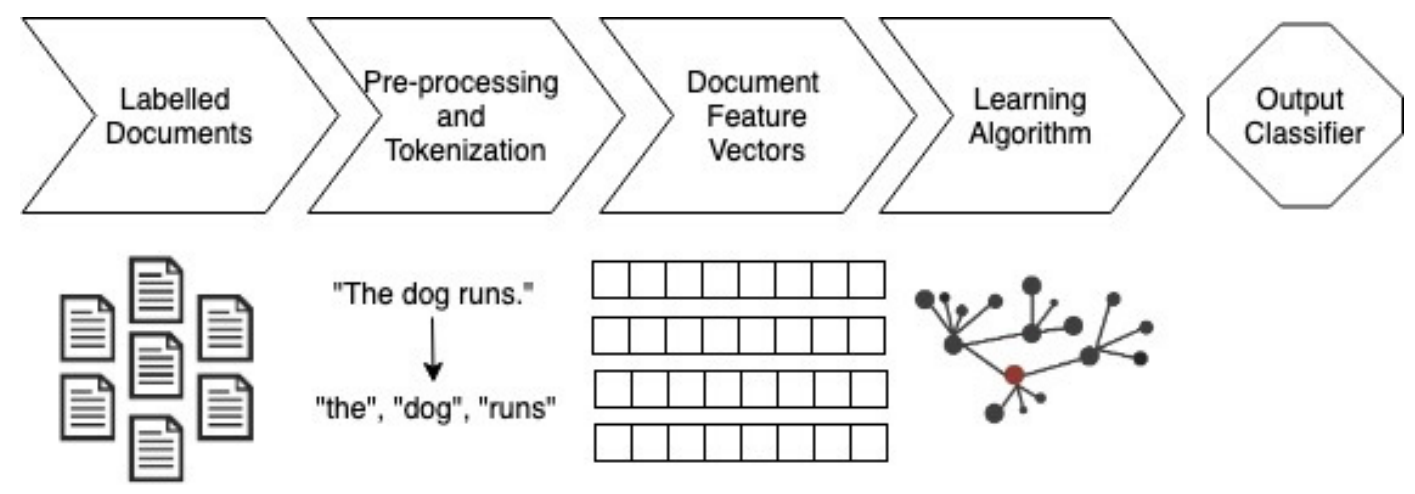

Figure 2.1: NLP pipeline for training a classifier

The following sections will review each step of this procedure for processing text from a "raw" form into a form that is suitable for input for classification algorithms. This section will first cover common text pre-processing methods used to standardize the incoming text into a "cleaned" form. Next, the document strings will be divided up into sub-strings, called tokens. After the documents have been transformed into a list of tokens, they can then be captured in a vectorized representation in numerous ways. It is possible to first form word vectors, and then process the word vectors into document vectors. Alternatively, one can proceed directly from the list of tokens to yield the document vectors. These vectors will represent each document as a data point in a high dimensional space, typically ranging from 100 to 1,000 dimensions. The given labels and the document vectors will be used to train an algorithm for the classification of future unseen documents. 


\subsubsection{Text Pre-processing and Tokenization}

Text pre-processing is the first step in the text classification pipeline, and can have a big impact on the final performance of the classifier [117]. The common steps of text pre-processing a text document into a clean, standardized form includes lowering cases of all characters, removing certain characters, stemming and lemmatizing words. The output of this process will be a list of tokens for each document. Tokens can then also be grouped into n-grams. Tokens are typically word based, but can also be at the sub-word level. Each of these steps are explained in more detail below.

1. Lowering case: First, all words will be converted to lower case, to remove duplicates of words with different capitalization, e.g., "The" would become "the".

2. Removing "stop words": Stop words are words that occur commonly without containing much meaningful information. Examples of these are: "a", "the", "again"... Once the words are all lower-cased, libraries can be used to determine which words are considered as stop words, and these can be removed from the list of tokens for each document.

3. Removing extra characters: Removing punctuation, numbers and symbols that are not part of words can also reduce the noisiness of the data-set, and this is often a step taken in pre-processing for text classification. Sometimes punctuation can be meaningful. For example, the use of exclamation marks can be useful in sentiment classification, and so the specific steps of this phase can vary from one application to another.

4. Stemming and Lemmatization: Finally, long words can be converted to stems or lemmas to preserve their meaning while reducing the vocabulary size and noise. Stemming involves truncating off endings of words, while lemmatizing is a more advanced approach, and, for example, can convert verbs to their root.

These steps can be taken to pre-process the text into a clean and standardized form. An example of using these steps to transform a sentence is shown in Table 2.1. 


\begin{tabular}{|c|l|}
\hline Original Phrase & $\begin{array}{l}\text { "There were } 100 \text { puppies in Ot- } \\
\text { tawa." }\end{array}$ \\
\hline Apply lowering case & $\begin{array}{l}\text { "there were 100 puppies in ot- } \\
\text { tawa." }\end{array}$ \\
\hline Removal of extra characters & "there were puppies in ottawa" \\
\hline Removal of stop words & "puppies ottawa" \\
\hline Stemming or Lemmatizing & $\begin{array}{l}\text { stemming: "puppi ottawa" } \\
\text { lemmatize: "puppy ottawa" }\end{array}$ \\
\hline
\end{tabular}

Table 2.1: Examples of text pre-processing steps.

\section{Tokenization}

Tokenization transforms a raw text document string into a list of "tokens". For example, the following sentence is shown in its raw text form and also in its tokenized form.

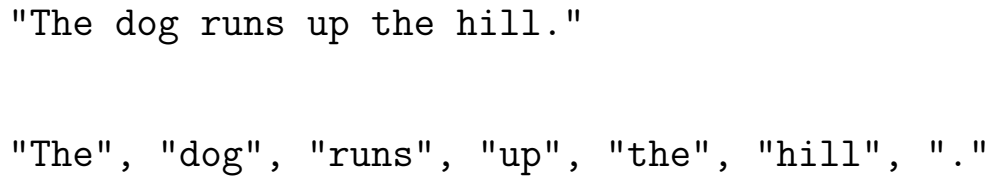

This is the most basic type of tokenization, where splitting is done based at the word level, and this can be achieved using simple regular expressions to detect the white spaces between the words. There are other more recent forms of pre-processing that involve sub-word tokenization [105]. A prominent example of this is referred to as WordPiece tokenization. This approach splits up words into subwords, and the word is then represented by a group of subwords. This is particularly useful to handle out-of-vocabulary terms in the test set of documents (words that were not present in any of the training documents). Another similar method is called SentencePiece, which is an unsupervised tokenizer that implements subword units such as byte-pairencodings (BPE), and brings various other subword tokenization techniques together into a single unified approach [56]. 


\section{N-grams}

Once the document is tokenized, n-grams can be constructed based on the orders of the tokens in the document's token list. Using n-grams permits the preservation of $\mathrm{N}$ tokens in a sequential order. For example, 2-grams, or "bi-grams" would mean preserving every two side-by-side tokens as an additional feature. These can be used, in addition to the single tokens, as features to represent the document. If the document is represented as a list of single, uni-gram tokens, it yields the most simple "Bag-of-Words" (BoW) representation, which will just count the frequency of these single tokens, and which does not preserve the order in any way. By using n-grams, one can preserve some of the syntactic meaning in the text. However, it can also greatly increase the number of features. The result of using the 2-grams in the above example sentence is shown below:

"The dog runs up the hill."

"The dog", "dog runs", "runs up", "up the", "the hill", "hill."

These bi-grams are based directly on the order of the words. However, it can also be done based on the order in the syntactic tree representation of the sentence. It is clear that by adding these additional features, one will obtain many more unique features across a group of documents, and thus the benefit of including this additional information into the text representation will also introduce the challenge of increasing the sparsity.

\subsubsection{Word Embeddings}

The feature representation of text is a widely-studied area, and there are many possible approaches which can be used to generate a numerical vector representation of text that can then be suitable for ML algorithms. One stepping stone on the way to generate document representations, is to first generate word or sub-word representations using word embeddings. Word embeddings learn features by mapping each token in the vocabulary to a n-dimensional vector. Word embeddings address the 
limitations of BoW models, by capturing the semantic meanings of the words in a reasonably low dimensional, dense, continuous space. Word embeddings map words into dense continuous spaces. For example "airplane", "plane", "aircraft", etc. are often used to mean the same thing. In the BoW model, these would each correspond to unique items in the vocabulary, adding unnecessary noise, and by also unnecessarily increasing the vocabulary size. In the word-embedding model, each of these words would be represented by very similar word-vectors as computed by some similarity metric, such as the cosine distance. A major benefit of using word-vector representations, is that it allows making use of the almost unlimited resources of unlabelled data available on the internet. Pre-training in an unsupervised way is very useful to encode meaning into the model that could not be obtained from labelled data alone, since the vast majority of existing text data is unlabelled. This pre-training allows downstream supervised tasks, such as text classification, to take advantage of all the information that exists in these large data sources to encode more meaning. This section will discuss three of the most-commonly-used word-embeddings: GloVe [84], Word2Vec [62] and FastText [12]. We will also discuss a more recent method, referred to as "Contextualized Word Vectors".

\section{GloVe}

The GloVe algorithm is unsupervised, and its training is performed on aggregated global word-word co-occurrence statistics from a corpus. The idea behind it is that the ratios of word-word co-occurrence probabilities have the potential for encoding some form of meaning. In the ratio of probabilities, noise from non-discriminative words cancel out, so that large values correlate well with properties that have specific relationships between the words, and the meaning associated with the concept of the word. The specific training objective of GloVe is to learn word vectors in such a manner that their dot product equals the logarithm of the words' probabilities of cooccurrence. The use of the logarithm enables the word representations to perform well on word analogy tasks as a ratio of logarithms. The ratio translates to a difference between two logarithms, and therefore resulting vectors can be added and subtracted in a linear way, and it simultaneously preserves the meaning [84]. 


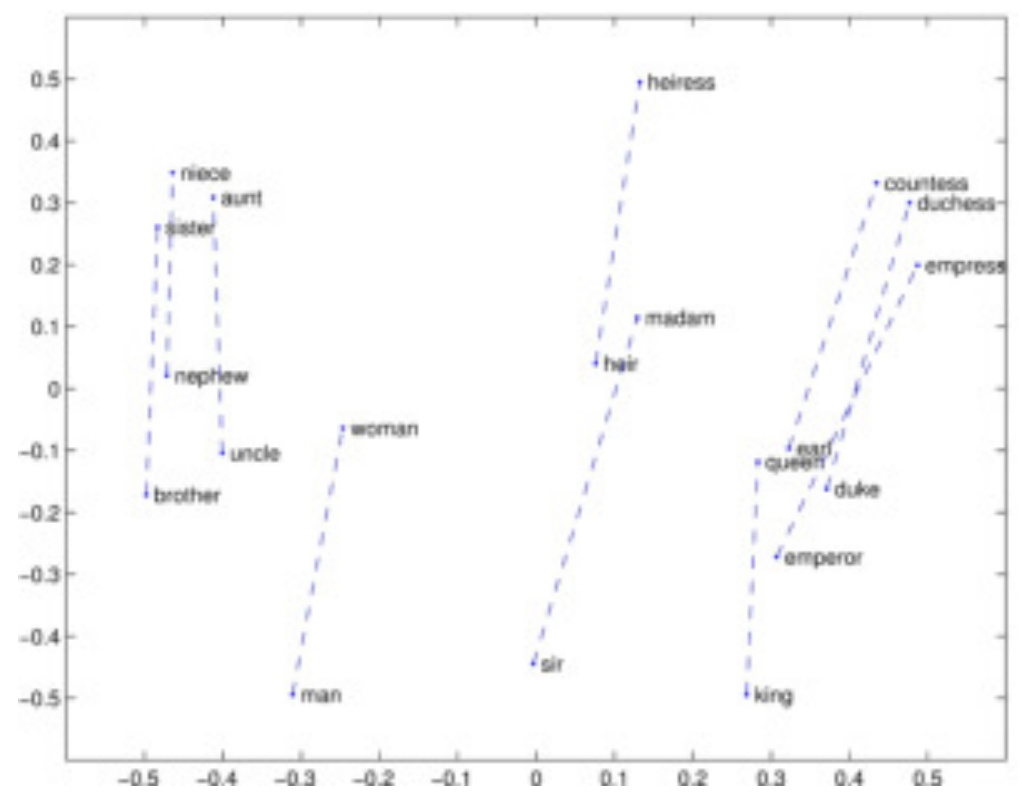

Figure 2.2: GloVe Relationship Representation [84]

The GloVe algorithm has been trained on large corpuses of text, such as Wikipedia, including 6 billion tokens, and the resulting representations have shown interesting linear substructures of the word vector space [84]. For example, the GloVe vectors are capable of capturing the relations between the words "man" and "woman", and have identified that this same relationship would be present between the words "king" and "queen". A visual representation that can illustrate this is shown in Figure 2.2, which shows the relationship between words projected onto two dimensions. It is amazing to see how it was possible to capture the concept of "gender", and how it was preserved in the vector representations of these words. Applying GloVe vectors enables representing words in a way that not only captures word similarities, but also some of the semantic meaning such as the actual relations between the words. Not only does this provide interesting results that identify relationships, but it is also able to achieve this in a fairly low-dimensional manner, by embedding vectors of sizes 50 to 300 dimensions, as opposed to typical vocabulary vectors which can have thousands of dimensions. 


\section{Word2Vec}

The Word2Vec [75] approach uses shallow NNs with two hidden layers, Continuous Bag-Of-Words (CBOW) and the Skip-gram model, to create a high dimensional vector for each word. The CBOW architecture predicts the current word based on the context, and the Skip-gram predicts its surrounding words based on the given current word. These vectors are also available in a pre-trained form, and there are various sizes ranging from 50-300 dimensions.

\section{FastText}

FastText, developped by Facebook, uses the skip-gram model. However, it involves an innovation in the pre-processing used, where each word is represented as a bag of character n-grams. For example, with $n=3$ the representation of the word "example" would be:

$$
\text { "ex", “exa", “xam", “amp", "mpl", "ple", "le" }
$$

This technique allows for the capturing of meaning related to the morphology of the words, and also has the advantage of being able to deal with out-of-vocabulary terms in the test data. [12]

\section{Contextualized Word Vectors}

A recent development in NLP that has lead to many state-of-the-art results, is called contextualized word embeddings. "Contextualized representations" is another word embedding technique that contains both the characteristics of the word e.g., syntax and semantics, and also the context. This can be accomplished by learning with a bidirectional language model trained by predicting the next word in both directions in a sentence. This technique was first introduced as a bi-directional Long Short-Term Memory (LSTM) [73], and then extended to create a deep contextualized word representation [85]. This method has been improved upon by the newest stateof-the-art model called "Bidirectional Encoder Representations from Transformers" (BERT),[24], developed by Google in 2018. It is a bi-directional transformer-based 
model that has achieved state-of-the-art results on numerous NLP tasks. This model is pre-trained on unsupervised problems including language modelling, as well as next sentence prediction. The output model can be fined tuned with training data for a wide variety of tasks.

\subsubsection{Document Vectors}

Feature representation of a text document is a widely-studied area, and there are many possible approaches that can be taken to generate a numerical vector representation of a text document for use in ML algorithms. The objective is to represent texts in a way that preserves the relationships and similarities within the content. The following section will provide some background on methods used to generate document vector representations. The main reported strategies include generating document vectors from word vectors (previously described in Section 2.2.2), as well as the more simple BoW model representations based on the vocabulary of the document, and they can be augmented by dimension reduction techniques that can be used on the very highdimensional vocabulary vectors.

\section{Document Vectors from Word Vectors}

Once the word vectors have been obtained from the texts, as described in Section 2.2.2, the next step is to generate a document embedding from the list of word vectors. There are several ways to approach this as shown in past studies. Some studies [76] have used the centroid of the word vectors to generate a document vector. Although this is a common approach, there are more recent advances that could yield an improvement on document representation. For example, the Google word2vec algorithm has a modification to enable generating a doc2vec through the same training procedure. Alternatively, another method called "sentence2vec" [44] takes into account word probabilities to generate a sentence vector from a list of word-embeddings. Another method, is called the Universal Sentence Encoder which can take variable length English text as input, and outputs a 512-dimensional vector. There are two variations of the Universal Sentence Encoder, that vary based in the architecture of 
the encoder [16]. One variation is based on the transformer, and the other is based on the Deep Averaging Network(DAN). The trade-off between the two variants is accuracy vs computational efficiency.

\section{Bag-of-Words Models}

The lists of tokens processed from the raw text can be used to directly represent the document as a vector. There are various ways to approach this, and the basis of most is the frequency of the tokens and features computed in each document. The most simple model of a document, the "Bag-of-Words" alluded to above, consists of the basic uni-gram feature representation the document, and ignores the semantic relationship between the words. This model can be extended to include other features, such as n-grams, syntactic patterns, etc., which are referred to as a "bag-of-features". In this type of model, a word or feature is encoded as a one-hot vector of the length of the full vocabulary. This means that in a vocabulary of 100 words or features, the word "dog" would be represented by a 100-dimension vector of 0s, with a 1 at the index corresponding to "dog". Some issues that can arise with this model, is that to capture enough meaning from the documents, the size of the vocabulary can easily extend into the millions. Also, since the order of words is not preserved, the sentences "Is this ok?" and "This is ok." would have the same vector representation. There are two main types of vectors that are commonly generated from the BoW representation: count vectors, Term-Frequency, Inverse-Document-Frequency (TF-IDF) vectors, and Boolean feature vectors. These will be described in more detail below.

- Count Vectors: One of the most basic ways to represent a document is by means of a count vector generated based on the counts of the occurrences of the words. This can be created out of the uni-grams or n-grams, and the result is every term or n-gram that occurs in all the documents corresponds to an index in the document vector. The result of this is that very frequent words will have much higher weights than other words, and will therefore have a larger influence on the results. This can negatively impact the performance if pre-processing is not done to carefully remove these frequently-occurring words. An alternative 
method that counters this issue is called "Term Frequency-Inverse Document Frequency" (TF-IDF), explained below.

- Term Frequency-Inverse Document Frequency (TF-IDF): The TF-IDF is a statistical measure used very commonly in information retrieval. It reflects how important a word is to a document in the context of a collection of documents. The value for a word is weighted based on how many times the word occurs in a given document, but it is then inversely proportional to the frequency of that word in the overall corpus. The effect of this is that the weights will be proportional to how specific or important a word is within a specific document (i.e., the value is offset by the fact that some words are just very frequently used overall) [109]. This index is calculated as:

$$
T F-I D F(t, d, D)=t f(t, d) i d f(t, D),
$$

where $t$ is the term, $d$ is the document, $D$ is the collection of documents, $t f$ is the term frequency, and $i d f$ is the inverse document frequency. This method possesses limitations, including the large size of the vocabulary, and the fact that words with similar meaning will still be represented by distinct indices.

- Boolean: These vectors are the most simple approach, with jut either 0 or 1 to represent the presence or absence of a word, and the counts are not taken into account.

The main issue with these models is the large size of the document vectors, which would typically be in the thousands of dimensions. Dimension reduction or feature selection approaches can be used to reduce the sizes of these vectors, which will be explained in the following two sub-sections, Section 2.2.3 and Section 2.2.3.

\section{Dimension Reduction}

As explained above, the approaches used to represent text involving n-grams and other features, can result in thousands to millions of features across a corpus of documents. Dimension reduction can, therefore, play a big part in making the data 
more manageable. Dimension reduction can also result in lower use of memory and lower time-complexity. The objective of dimension reduction is to preserve as much meaning as possible from the original full data-set, while reducing the size. Some commonly-used approaches for this are principal component analysis, random projections, non-negative matrix factorization, auto-encoders and t-distributed stochastic neighbour embeddings [54].

\section{Text Feature Selection}

Another way to reduce the size of the features, is to use feature-selection to select the best $\mathrm{k}$ features. The effect of this is, typically, to obtain more interpretable models, than building a model out of vectors resulting from dimension reduction. The latter is usually a combination of multiple features in each of the resulting new features. Some commonly-used approaches to select the best features, are by using filters, wrappers, and embedded models [23]. These approaches are explained in the following list:

- Filter models: This approach evaluates each feature with a scoring function, and retains the top $k$ features. The scoring function evaluates how good the feature is, and this could be calculated based on the TF-IDF, the mutual information, information gain, the $\chi^{2}$ metric, the correlation, the result of a t-test, or based on similar criteria.

- Wrapper models: This approach uses a search strategy to evaluate possible subsets of features, and computes how they affect the performance of the classifiers. These methods achieve better performance than filter methods in classification tasks; however, the computational complexity is much higher.

- Embedded models: This approach embeds feature selection into the training of the classifier. For example, Decision Trees are well-known methods that select features with the best discriminative power. 


\subsubsection{Summary}

The main steps required to prepare a raw text document for classification includes: pre-processing, tokenization, and creating a document vector representation. There are many variations of pre-processing and tokenization methods available that can lead to large impacts on the performance of down-stream classification tasks. Word embeddings can be used as a method to represent tokens or words, that can take into account information available in the massive resources of information available on the internet. These word-embeddings can then be used to construct document or sentence embeddings. Document vectors can also be created with simple BoW approaches, though, typically, these methods will also require subsequent steps of feature selection or dimension reduction to preserve as much information as possible in a more efficient way. Once the text has been transformed into a set of labelled vectors, these vectors can then be used in classification models, as will be described in the next section.

\subsection{Supervised Classification}

Classification consists of predicting the class of new samples, using a model inferred from the training data. In this section, we will introduce a few of the existing text classification algorithms. There are many variations of learning models for classification from unsupervised to fully-supervised methods, including semi-supervised, active learning, etc. This thesis focuses on supervised learning, and so models relevant to this scope and which are suitable for text classification will be discussed in the following sections.

\subsubsection{Text Classification Problem Description}

In text classification, we assume that we are working with a document $d \in X$, where $X$ is a document space. $d$ could have a label in $C=\left\{c_{1}, \ldots, c_{m}\right\}$, consisting of $m$ possible class labels. The document space $X$ will, typically, be very high dimensional, represented by many features from the text, such as a vocabulary of words. The 
classes will be defined by a human, for the needs of their applications, for example a sentiment, or type of content in the document. The documents and the given labels will be jointly referred to as a labelled training set.

Using a learning algorithm, the objective is to learn a classification decision function that maps the testing documents to classes. The learning method takes the training set as input and returns the learned classification decision function.

\subsubsection{Text Classification Methods}

This section will discuss classification methods specific to text data. Text data requires unique considerations when it concerns classification, related to both the nature of the noise present in the labels, and the types of models that can be effectively used in text classification. Some attributes of text data that contribute to its unique requirements are as the following:

- High-dimensional data: Usually text is represented in a high dimensional space of features, as explained previously;

- Sequential models: Text data is inherently sequential, and certain models are better suited for capturing this structure;

- Subjective labels: Often, tasks related to text classification, such as sentiment analysis, involve annotators who are given fairly subjective annotation guidelines. For example, what one person perceives as "happy", could be perceived as "excited" by someone else in a written text;

- Large amount of data available: Massive amounts of unlabelled data is available, and can be used to build language models. Transfer learning approaches can be applied to tune those trained models to a specific classification task.

Commonly-used classification algorithms includes: Logistic Regression, Naive Bayes, Support Vector Machines (SVMs), Decision Trees, and k-Nearest Neighbours (kNNs). These models will be discussed in detail in the following section, including specific aspects about their respective models, and their respective specific limitations and 
benefits. In the following sections, each of these models will be reviewed, as well as a few more-recently developed methods in text classification, including recurrent neural nets and transfer learning approaches.

\section{Logistic Regression and Naive Bayes}

The most commonly-used simple classification models are Logistic Regression and Naive Bayes. These models adopt the assumption that the value of a particular feature is independent of the value of any other feature. In the context of text classification, the assumption is that the probability of each word or feature appearing in a document is independent of the occurrence of any other word in the same document. The decision boundary for both these methods is linear, and techniques can be used to transform the data to accommodate non-linear class boundaries in the original space. However linear boundaries, typically, perform well for high-dimensional feature spaces, such as in text classification. A limitation of high-dimensional feature spaces for these models arises when the number of features is higher that the number of training examples, in which case the training would result in the model memorizing the training set, instead of extracting knowledge from it [4]. It is important to consider regularization to mitigate this effect. The main difference between the two models is that Logistic Regression is a discriminative model and Naive Bayes is generative. These two methods are both very commonly-used in text classification and will be explained in detail in this section.

Logistic Regression is a discriminative model, meaning that it directly optimizes the probability $p(y \mid x)$, where $y$ is the class label and $x$ is the observed data. Logistic Regression is a modification of linear regression, by substituting the hypothesis function of a linear mapping, to be the sigmoid function. The use of the sigmoid function for hypothesis assignment results in outputting values between 0 and 1 , which can then be translated to either 0 or 1 based on a threshold (of 0.5 ) for the binary classification task. The hypothesis with the sigmoid function is:

$$
\begin{gathered}
p(y=1 \mid x)=h_{\theta}=\frac{1}{1+\exp \left(-\theta^{T} x\right)}, \\
p(y=0 \mid x)=1-h_{\theta} .
\end{gathered}
$$


To derive the parameter, $\theta$, one uses the maximum likelihood estimation method. Training a model to find the parameters can be done by maximizing the conditional log likelihood of the training data. The log is used to ensure that the cost function will be convex, with a single global optimum point. The Logistic Regression cost function is:

$$
\operatorname{Cost}\left(h_{\theta}(x), y\right)=y *\left(-\log \left(h_{\theta}(x)\right)\right)+(1-y) *\left(-\log \left(1-h_{\theta}(x)\right)\right) .
$$

For a training set with $m$ points, the likelihood of the parameters is:

$$
L(\theta)=p(Y \mid X ; \theta)
$$

where, the $L$ represents the likelihood, and the $Y$ and $X$ represent the class and data for all the points in the training set. Using the chain rules for probabilities and the previously given equation for $p\left(y^{i} \mid x^{i} ; \theta\right)$, this equation can be written as:

$$
L(\theta)=\prod_{i=1}^{m}\left(h_{\theta}\left(x^{i}\right)^{y^{i}}\right)\left(1-h_{\theta}\left(x^{i}\right)\right)^{1-y^{i}} .
$$

This can be transformed into the log likelihood:

$$
l(\theta)=\log L(\theta)=\sum_{i=1}^{m} y^{i} \log \left(h_{\theta}\left(x^{i}\right)\right)+\left(1-y^{i}\right) \log \left(1-h_{\theta}\left(x^{i}\right)\right) .
$$

The objective will be to maximize the log likelihood, and this can be done iteratively using an optimizer such as the gradient descent towards the global optimum. Thus, to update the parameters at each step up the gradient, one uses update equation:

$$
\theta:=\theta+\alpha \nabla_{\theta} l(\theta)
$$

By taking the gradient of the log likelihood function, this equation can be simplified to:

$$
\theta:=\theta+\alpha\left(y^{i}-h_{\theta}\left(x^{i}\right)\right) x_{j}^{i}
$$

The Naive Bayes is a generative version of this model, where instead of optimizing the conditional probability, it attempts to optimize the joint probability of the labels 
and the data that was observed, $P(Y, X)$. This model will still yield a similar linear boundary as its decision boundary. The Naive Bayes can model the document as a binary vector, referred to as the multivariate Bernoulli Naive Bayes, or as a vector of term frequencies, in which case it is called multinomial Naive Bayes [71].

\section{Support Vector Machines (SVMs)}

SVMs, originally developed in 1995 [21], classify data into different classes by finding a line or hyperplane which separates the training data set into classes. As there are many possible hyperplanes, the SVM algorithm tries to maximize the distance between the various classes that are involved, and this is referred to as margin maximization. The hyperplane that corresponds to this maximized margin distance, $d$, is the chosen hyperplane for the decision boundary. This is a well-defined convex optimization problem, with a global optimum. A key concept of SVMs is the "support vectors", which are the points that lie closest to the decision surface. These are points that would change the position of the decision boundary if they are removed. The idea behind SVMs, is that the decision boundary should be influenced by important points, the support vectors, and not affected by other points further from the decision boundary.

SVMs can also be extended to non-linear boundaries using kernels to transform the data into higher-dimensional spaces [104], although linear kernels are most commonlyused in text related applications.

The equations that describe the basic binary SVM are as follows. Let the data be represented as $\left(x_{1}, y_{1}\right), \ldots,\left(x_{n}, y_{n}\right)$, where $\left\{y_{i}\right\}$ are the binary class labels that indicate which class each data point belongs to, and $\left\{x_{i}\right\}$ are the points in a ddimensional space. If the binary class labels are 1 and -1 , then a hyperplane between the two groups could be defined as: $w x-b=0$, where $w$ is the normal vector to the hyperplane. If the training data is linearly separable, then this constraint can be satisfied, $y_{i}\left(w x_{i}-b\right) \geq 1$, for all $i$. This can be used to formulate the optimization problem to maximize the gap on either side of the hyperplane, as minimizing $\|w\|$, with $w$ being subject to that constraint.

In the commonly occurring case that the data is not linearly separable, the binary 
classifier optimization would be formulated as shown in Eq. (2.1). The objective would be to minimize Eq. (2.1), where the $\lambda$ is used to allow a trade-off between increasing the margin, and ensuring that $x_{i}$ lie on the correct side of the margin:

$$
\left[\frac{1}{n} \sum_{i=1}^{n} \max \left(0,1-y_{i}\left(w x_{i}-b\right)\right)\right]+\lambda\|w\|^{2} .
$$

This equation is clarified in Figure 2.3.

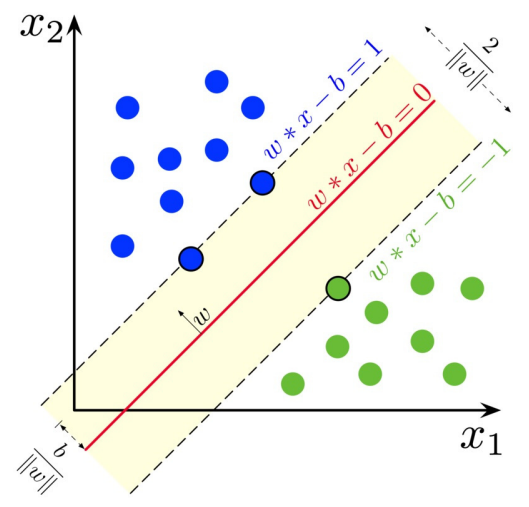

Figure 2.3: Visualization of SVM's equations [18]

Some strengths of SVMs are that they are less likely to overfit, and they also generalize better on unseen test data [8], and a limitation is their simple representation that may be unable to capture nuanced patterns that may arise. Overall, the model is fairly similar to the Logistic Regression and Naive Bayes models previously discussed.

\section{k-Nearest Neighbours (kNN)}

$\mathrm{kNN}$ is a non-parametric classification method that has a decision boundary quite different from those previously described. Given a test document $x$, the kNN algorithm finds the $\mathrm{k}$ nearest neighbors of $x$, i.e. the $\mathrm{k}$ closest documents based on some distance measure in the high-dimensional feature space, and assigns the category of $x$ based the classes of those $k$ neighbors. The parameter $k$ is chosen by the user. When deciding which document is the nearest neighbor to $x$, one needs to evaluate each of the documents according to some distance or similarity measure. There are many possible distance metrics that could be used for this, including the Euclidean, cosine, and the Hamming distance. 
This method is easy to implement and its performance is reasonably good, especially for data with non-linear class boundaries. Another advantage of the kNN, is that it is adaptable to model rare classes, with a high class-imbalance. A major drawback of the kNN method is its high computational cost, since it is a "lazy" learning method. Each time a text document is given, the $\mathrm{kNN}$ method needs to re-examine the whole dataset to find the $\mathrm{k}$ nearest neighbors for the object, and compute the similarity metric for each. This method also suffers from the curse of dimensionality, requiring many more training examples than features, and it is prone to over-fitting

\section{Decision Trees}

A Decision Tree is a well-known ML algorithm that has been used extensively in automatic classification tasks [89]. When applying it to text categorization tasks, it selects informative words or features according to the information gain criterion, and performs a hierarchical decomposition of the data space. Given a document to be classified, the constructed Decision Tree is used to predict which category the document should belong to, according to the occurrence of word combinations in the document. An advantage of Decision Trees, is that they are very fast for both prediction and learning. Also, Decision Trees are highly interpretable models. A disadvantage is that decision trees do not perform as well on high-dimensional data. The method of optimizing the Decision Tree is very important for the performance. Their main disadvantage is that they are also extremely sensitive to small perturbations in the data, and can easily over-fit, though there are advancements possible to address these issues, including pruning and validation methods. The performance of the Decision Tree can also be greatly improved when integrated with ensemble models, most commonly known as Random Forests. However, this increase in performance also comes with an increase in computational cost. Ensemble-based trees make use of boosting and bagging techniques to combine more than a single classifier that employs different decision rules [51]. 


\section{Ensemble Models}

Ensemble models are very popular techniques, and combine the outputs of predictions of many individual learners to determine the final prediction. There are many types of architectures for ensemble models, and we will discuss some of the most common ones.

The most common and straight-forward ensemble method is an extension of Decision Trees, called Random Forests. A Random Forest uses a numerous randomized, uncorrelated Decision Trees, and each Decision Tree casts a vote to predict the class of the test example. The final prediction is chosen based on the majority of the votes. This procedure is called "Bagging". The effect of using multiple Decision Trees on a randomly drawn subset of features improves results over individual Decision Trees by preventing over-fitting. This method also becomes more robust against noise and outliers. Besides, it also has a benefit similar to Decision Trees, namely that of being able to learn a hierarchical, complex decision boundary and to model non-linear boundaries and complex interactions between the features. Increasing the number of Decision Trees to be used in the ensemble can improve the overall performance, but it can also result in a higher computation time.

In general, the main techniques involved in ensemble models are called Bagging, Boosting and Stacking. Bagging, as is used by Random Forests, is the process of creating random sub-sets of the training data, building a classifier for each sample, and combing the results with majority voting. Bagging helps to reduce the variance error. Boosting, commonly-used in the algorithms Adaboost and gradient boosting, can improve ensemble models by taking into account the sequential learning of the predictors. The training examples chosen for each iteration of training are based on the performance of the previously-trained classifier, giving higher weights to the previously mis-classified observations. Boosting can lead to a better performance than bagging, but also has a higher tendency to over-fit. Stacking involves two phases, the first of which is to learn base learners, and next involves learning a second phase based on their combined predictions. All the three of these methods can reduce the variance, bias and improve the predictions and model's robustness, over that of a single classifier. 


\section{Neural Networks}

Artificial Neural Networks (ANNs) are constructed from a large number of connected artificial neurons. Many different types of Neural Network (NN) approaches have been used for document classification tasks. Some of these are simple, such as the single-layer perceptron, which contains only an input layer and an output layer. The multi-layer perceptron, which includes one or more hidden layers in its structure, is also commonly-used. As common text classification problems represent linearly separable classes in high-dimensional space [2], single-layer ANNs are most frequently applied.

However, the structure of the ANN used is very flexible and can learn subtle patterns in the text. Recently Convolutional Neural Networks (CNNs) have been used for text categorization [48], by taking into account the internal structure of the documents, including the order of the words. This type of model is particularly good at handling documents with high-dimensional features, and documents with noisy and contradictory data. The drawbacks of this model is the high computing cost and high memory usage. Another disadvantage is that the ANNs are less interpretable.

Traditional ANNs fail to incorporate sequential information. This issue has been addressed with the use of more sophisticated NN architectures, such as Recurrent Neural Networks (RNNs). These incorporate feedback loops to simulate the persistence of the sequential information. The LSTM model, or "Long Short Term Memory" NN model, is a variant of the RNN model that is particularly suited to tasks such as semantic learning. They are capable of learning a phenomenon referred to as "longterm dependencies", which are connections between a given context and word that bear a larger-than-usual gap in logic, i.e., they could be separated by several words or even phrases in the sequence. LSTMs are specially designed in a way that stores information for a long period of time as may be needed to preserve the contextual meaning. 


\section{Transfer Learning Models}

Current state-of-the-art methods in text classification rely on transfer learning. Because there are vast resources available of unlabelled text, models can be pre-trained to encode the meaning present in this data. These pre-trained models can then be used as the basis for supervised tasks such as text classification. This can be done by either first training a text representation using a language model trained on large unlabelled datasets, so as to use as the input representation to the classifier, referred to as word vectors (described earlier in Section 2.2.2). There are also models that can be "fine-tuned" by updating the weights of the complete model that was originally trained on the unlabelled data. It can be fine-tuned by adding a new final output layer specific to the task, and back-propagating through the full network to update the weights. This has the effect of "contextualizing" or modifying the text representations for the given task based on the labelled data-set, as described in Section 2.2.2. Two recent methods that have become very successful using this approach are known as BERT ("Bidirectional Encoder Representations from Transformers")[25] and XLnet [121].

\subsection{Learning Automata (LA)}

The following section will discuss the field of LA, first presenting an overview of the field and and providing an explanation of fundamental concepts. It will thereafter, discuss details of specific types of LA, and finally, discuss their applications in classification, and in particular, on how they could be used for text classification. The following survey will be necessarily brief, and more detailed reviews can be found in several books and articles [77, 78, 81, 122].

\subsubsection{Overview of LA}

LA have been studied since the 1960s, since they were first proposed by Tsetlin, with the invention of the Tsetlin LA [114]. The field of LA is the pre-cursor to the area of 


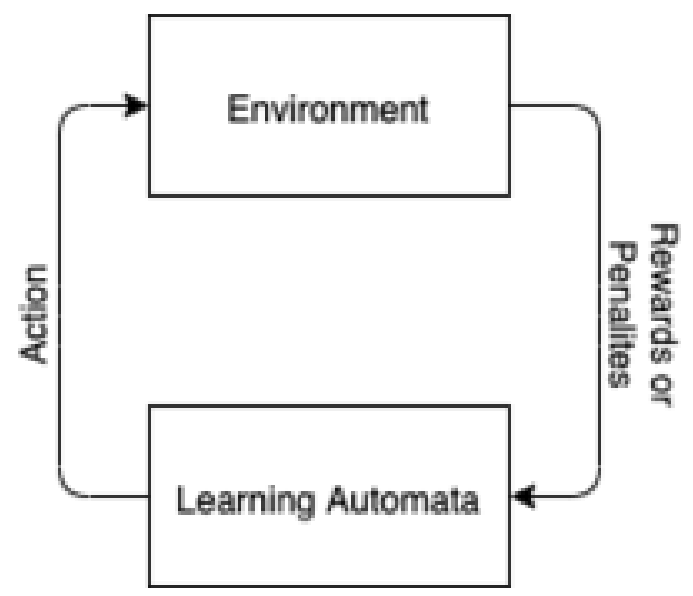

Figure 2.4: Overview of general LA learning model

Reinforcement Learning. Whereas LA do not model the Environment, in Reinforcement Learning, one attempts to also model the state in which the Environment is. With respect to the current status of Reinforcement Learning, LA are characterized as policy iterators, and are updated strictly based on the response from the Environment. LA are adaptive units that can make decisions about the identity of the actions that are to be chosen in the specific domain. LA units can learn the best action to be chosen in an Environment, which is typically a random (or stochastic) environment. Such an Environment will respond randomly to the LA unit's actions by giving feedback of rewards or penalties. The LA's objective is to learn how to pick the best action based on this feedback. The best action is the action that will result in the most rewards and least penalties from the Environment, in the expected sense.

An overview of the general stochastic learning model is shown in Figure 2.4. The LA learns through the feedback loop, that involves choosing an action, obtaining a response from the Environment, and then changing its state accordingly, after which it chooses another action based on its current state.

Each component of the learning model shown in Figure 2.4 will be explained in further detail in the following sections. 


\section{The Environment}

The Environment could be any entity that provides feedback to the LA unit, for example, real time systems with sensors. The Environment will give a response back to the LA after each action that the LA takes, as characterized by the feedback loop in Figure 2.4. In a Random Environment, the response from the Environment is determined by a random function. The Environment, can be formally defined as in Eq. (2.2):

$$
\mathbb{E}=\{\underline{\alpha}, \underline{c}, \underline{\beta}\},
$$

where $\underline{\alpha}$ is the set of possible actions, $\underline{c}$ is the set of penalty probabilities corresponding to each action in $\underline{\alpha}$, and $\beta$ is the set of feedbacks possible from the Environment, which are typically 0 for reward and 1 for penalty. The penalty probabilities, $\underline{c}$, dictate how likely it is that the Environment will provide a feedback response of a penalty for each action. Specifically, for each action $\alpha_{i} \in \underline{\alpha}$, there is a corresponding penalty probability $c_{i} \in \underline{c}$, which is defined as:

$$
c_{i}=\operatorname{Pr}\left[\beta(n)=1 \mid \alpha(n)=\alpha_{i}\right],
$$

where $i \in(1, \ldots, r)$ correspond to the specific actions possible, when there are a total of $r$ actions that could be taken by the LA, and $n$ is the current time step.

In applications to text classification, the Environment would be the class labels for the text documents to be classified. The noisiness of the feedback from the Environment in the application to text classification is due to the fact that, often, human annotators can make errors during the document annotation process, as will be explained further in the literature review section on noisy labels, in Section 2.5.

\section{Definition of LA}

A LA is formally specified by:

$$
\{\underline{\phi}, \underline{\alpha}, \underline{\beta}, F, G\},
$$

where $\underline{\phi}$ represents is the set of states and $\phi(n)$ is the current state after time step $n, \underline{\alpha}$ is once again the set of actions that the automaton can pick from, $\underline{\beta}$ is the set 
of possible feedbacks where $\beta \in\{0,1\}, F$ represents the transition function for the LA from the current state to the next state, and $G$ is the function that specifies the selection of an action by the automaton. The transition function is formally specified as $F(\phi(n), \beta(n))$, and the function $G$ is formally specified as $G(\phi(n))=\alpha(n)$.

The probability of the LA choosing an action given the current time instant, is defined by Eq. (2.4).

$$
p_{i}(n)=\operatorname{Pr}\left[\alpha(n)=\alpha_{i}\right]
$$

where $p_{i}(n)$ is the probability of the LA choosing $\alpha_{i}$ at time $n$.

\section{LA Actions}

LA attempt to learn the optimal "actions" through repeated interactions with the Environment through the process shown in Figure 2.4. This is similar to training a supervised learning algorithm. The actions are chosen according to a probability distribution, which is updated based on the Environment's responses. An example of an action in a real world control system would be that of taking the action to increase or decrease the power to a heater, given the response feedback from a temperature measurement. In the application to text classification, the action could be to include or exclude a certain word as a feature, as is done in the recent study by Berge et al. [9]. It could also be to select an optimal point as a decision boundary, as is done in a study by Sastry et al. [101], where a continuous action LA was used for classification.

\subsubsection{Types of Learning Automata}

In this sub-section, we submit an introductory overview of several types of LA.

\section{Tsetlin Automata}

Tsetlin automata are Fixed Structure Stochastic Automata (FSSA) that can decide on the action chosen in a stochastic environment by using its state information, and by changing its state based on the responses from the Environment. For the action, $\alpha_{i}$, the Environment triggers either a reward response with probability $1-c_{i}$ or penalty 
with probability $c_{i}$. The mechanism behind the Tsetlin automata is shown in Figure 2.5 for a two action Environment with actions $\alpha_{1}$ and $\alpha_{2}$.

Figure 2.5: Transition map of the Tsetlin Automata on receiving a reward
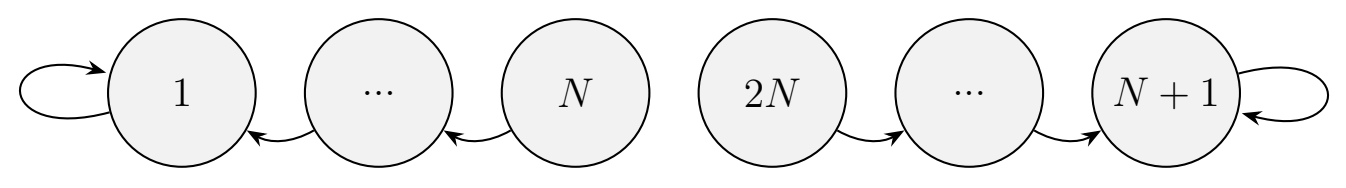

Figure 2.6: Transition map of the Tsetlin Automata on receiving a penalty

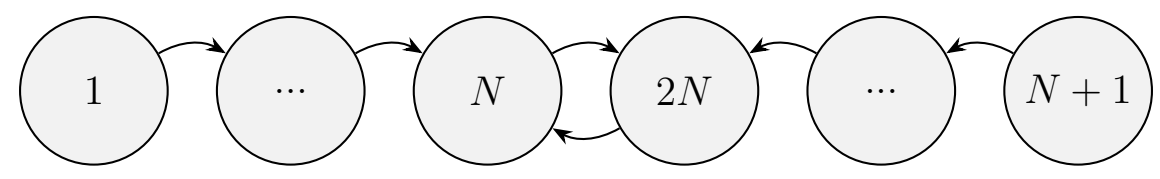

The circles in the diagram represent the states of the automata. The current state dictates which action the automata chooses, either $\alpha_{1}$ or $\alpha_{2}$. If the automata is in the left half of the states, i.e., $\phi(n)=1 \ldots N$, then it chooses $\alpha_{1}$, and alternatively, if it is in the right half of the states, i.e., $\phi(n)=N+1, \ldots, 2 N$, then it always chooses $\alpha_{2}$. The learning of the LA is made possible by the mechanism of transitioning from state to state. One set of transitions occur if the automaton is given a reward from the Environment, resulting in the automaton moving further into the state of the current action. If the Environment gives a penalty, the automaton will follow the corresponding transitions, which results in it moving towards the center and towards the other action's states. The Tsetlin LA can be proven to be " $\varepsilon$-optimal" in certain random environments, where " $\varepsilon$-optimal" is a term formally defined in the literature [74], meaning that it will converge to the optimal action as it iterates through its feedback loop with a probability that is arbitrarily close to unity.

After the invention of the Tsetlin LA, other FSSA were developed, including the Krinsky LA and the Krylov LA. Krinsky's LA design has a different mechanism from the Tsetlin LA for when it receives a reward, which is to jump directly to the most internal state. The result of this mechanism is that the Krinsky LA is $\varepsilon$-optimal in all Random Environments. The Krylov LA differs from the Tsetlin LA when it receives a penalty. When a Krylov LA receives a penalty, it will move towards the internal 
state with probability 0.5 , and it will move outward as if it received a reward with probability 0.5 . The Krylov LA has also been proven to be $\varepsilon$-optimal in all Random Environments.

$L_{R I}$

The $L_{R I}$ learning scheme is described in the following equation, where $k_{r}$ is the parameter for reward, $\alpha(n)$ is the action chosen at time $n, \beta(n)=1$ when a penalty is received, and $\beta(n)=0$ when a reward is received at time $n$ :

$$
p_{i}(n+1)= \begin{cases}k_{r} p_{i}(n)+\left(1-k_{r}\right) & \text { if } \alpha(n)=\alpha_{i} \text { and } \beta(n)=0 \\ p_{i}(n) & \text { if } \alpha(n)=\alpha_{i} \text { and } \beta(n)=1 \\ k_{r} p_{i}(n) & \text { if } \alpha(n) \neq \alpha_{i} \text { and } \beta(n)=0 \\ p_{i}(n) & \text { if } \alpha(n) \neq \alpha_{i} \text { and } \beta(n)=1 .\end{cases}
$$

This equation is used to update the probability vector for choosing actions at each step. It can be noted that when penalties are received, the vector does not change. This equation applies for any number of actions, though in this case we consider the two-action $L_{R I}$, where $i \in\{1,2\}$. This learning scheme is absolutely expedient and $\varepsilon$-optimal, terms defined in the literature [74], and it thus will converge to the action, with a probability as close to unity as $k_{r} \longrightarrow 1$, as time proceeds.

\section{Discretized Automata}

A Discretized LA can also be used to achieve the learning, such as the Discretized $L_{R I}$ or the Discretized $L_{R P}$. The Discretized version of the $L_{R I}$ can increase the rate of convergence by reducing the slow asymptotic approach to the optimal action. Due to discretizing the probabilities associated with choosing each action, the final steps are equal in size to the sizes of steps early on, instead of decreasing in magnitude by a factor of $k_{r}$ at each step. This can be modelled as a Markov chain with each node corresponding to a discrete probability of choosing an action. The resolution of the $D L_{R I}$ depends on the number of nodes or segments of the probability range chosen. For example, with $N=4$, the possible probabilities for each action are 
$P_{i} \in\left\{0, \frac{1}{3}, \frac{2}{3}, \frac{3}{3}\right\}$, and the transitions are made to these different states based on the current state and the response from the Environment.

\section{Estimator Scheme}

Estimator schemes can also be used to solve the problem under consideration, such as the "pursuit algorithm". This method is very similar to the $L_{R I}$, except that instead of increasing the probability of the chosen action being rewarded, the probability increase is always given to the currently estimated best action. The currently estimated best action is determined by which of the actions has received the most rewards so far, and these are new parameters that must be tracked throughout the course of the learning process. This method has been shown to achieve a higher accuracy than the $L_{R I}$ in shorter time, and these families represent the state-of-the-art rates of convergence, and have been proven to be $\varepsilon$-optimal.

\subsubsection{Automata for Text Classification}

Recently, LA, and specifically the Tsetlin automata, has been applied to text classification [9], specifically for text data on medical records, IMDb datasets, and the 20 newsgroups dataset. The objective of the authors was to be able to have a classifier with a good performance, that also possessed a reasonable level of interpretability and computational complexity. The Tsetlin machine was used to represent the terms of a text as propositional variables. An example from the paper, given to illustrate this idea is:

if rash $\wedge$ reaction $\wedge$ penicillin then Allergy.

The representation of the document used was bit-vectors of the length of the vocabulary for each document, with a ' 1 ' indicating the presence of a term, and a ' 0 ' indicating the absence of the term in the document. The scheme used the Tsetlin machine for choosing the significance of the terms, as described below:

1. A new document arrives and is characterized by the pair $(X, y)$, where $X$ is the document bit vector that represents the presence or absence of terms. For 
example, if $x$ "penicillin" is 1 , it indicates the presence of that term. Also, $y$ is the category label given to the document.

2. Each term has a two designated Tsetlin automaton, one with positive polarity for the term, and the other if the term is to be negated. Each Tsetlin LA decides whether to include or exclude its designated term, based on the state it is in. This leads to a new configuration of clauses $C$, where $C_{j} \in C$, and $C_{j}=x_{q_{1}} \wedge \ldots \wedge x_{q_{r}} \wedge \neg x_{q_{r+1}} \wedge \ldots \wedge \neg x_{q_{s}}$, where the negated terms are denoted as $\neg x_{i}$.

3. Each clause $C_{j} \in C$ is evaluated with the document bit vector, $X$, as input.

4. The output of the Tsetlin machine is the predicted label, $y$. This output is obtained by thresholding the function that adds up the evidence from the clauses. The evidence is added up by summing the terms with positive polarity, and subtracting the negated terms that are present in the document:

$$
f(X)=\sum_{j-1,3, \ldots}^{m-1} C_{j}(X)-\sum_{j-2,4, \ldots}^{m} C_{j}(X) .
$$

5. The predicted label is compared to the true label, on whether it is correct or not. Based on these, rewards, penalties, or inactions are given by the Environment, and distributed to the Testlin LA. Based on each reward or penalty, each Testlin LA will update its state as described in the previous section on the Tsetlin LA.

6. These steps are repeated for each document in the training set of labelled documents.

This is a novel method. However the paper showed that its performance was comparable to other simple text classification models described in the previous section. It is possible that this method will be robust to noise in the class labels, since LA, such as the Tsetlin machine, are able to learn well in random environments.

This method also works with multi-class classification, since the sum of total scores for each class is used to compute the final predicted class. Indeed, this method can be used to learn from any number of classes. 


\subsection{Classification with Noisy Class Labels}

Classification consists of predicting the class of new samples using a trained classification model. Label noise refers to the situation when each training sample has an observed label that is subjected to noise before being presented to the classification algorithm.

\subsubsection{Overview of Noise in Labels}

Label noise is the result of a stochastic process. Error in labels created systematically will not be considered as label noise. Also, labelling errors are assumed to be independent. A fully specified model of label noise is currently not available, and so we need classification algorithms that can also deal with label noise. In these situations, we cannot live with the assumption that labels are perfect, and that they represent ground truth. Thus, learning in this situation can be called "imperfectly supervised", which is in one sense, in between supervised and unsupervised learning.

Approaches to deal with label noise are related to other ML tasks, including outlier detection and anomaly detection. Many methods developed to deal with these tasks can also be used and tested for applications related to label noise. The connection between these tasks is as follows: mislabelled instances may actually be detectable outliers and as anomalies in the data when the incorrect label has a low probability of occurring. However, there are also many cases where the label noise occurs close to the class boundaries, in which case these data points would not present themselves as outliers or anomalies, and consequently, specialized methods, specific to label noise, are required.

\section{Cause of Noise in Labels}

Mistakes in labels can be caused by many factors, and it is important to consider the possible sources for the specific data-set and the application of the classifier. Overall, label noise occurs naturally due to human error during the annotation process. Even expert annotators are prone to making mistakes [41,69]. There are several factors that can contribute to increasing the noisiness of labels. The first is when the criteria given 
to the human annotators are not clear, or when they are too subjective [13, 67, 108]. Another cause is that of using non-experts for the task, such as crowd-sourcing. Finally, miscellaneous errors can accumulate, such as accidental clicks. The fact that annotations is done by humans will always result in a margin of human error in the output annotations.

\section{Types of Noise}

The main classes of noise most frequently studied and discussed in the literature are adapted from the types of missing data, created by Schafer and Graham [103]. These types of label noise are shown in the following list. When discussing noise, $X$ will refer to the vector of features, $Y$ will refer to the true labels for each instance, $\tilde{Y}$ is the observed labels after noise, and $E$ is a binary variable indicating whether a label was flipped or not (i.e., $Y \neq \tilde{Y}$ ).

- Label Noise Completely At Random (NCAR): This type occurs when the noise is independent of the true class and independent of the values of the data. This can be referred to as the error rate [63], $p_{e}$. When this quantity, $p_{e}$, approaches 0.5 , the labels become useless since they have lost too much information [11]. In the multi-class classification situation, the incorrect label chosen will be chosen at random out of all the possible incorrect classes, and this is called the "uniform label noise" model.

- Label Noise At Random (NAR): This type occurs when the noise depends only on the true label. It commonly occurs in situations when some classes are more likely to be mislabelled than others. This means, for example, in document classification, if one category of documents is too abstract and is not well understood by the annotators, they would likely be making mistakes more often on that label. This type of noise can be characterized by the following probability:

$$
P(\tilde{Y}=\tilde{y} \mid Y=y)=\sum_{e \in\{0,1\}} P(\tilde{Y}=\tilde{y} \mid E=e, Y=y) P(E=e \mid Y=y) .
$$


In the case of multi-class labels, the probabilities can be represented by the following labelling, or transition matrix, where the total number of classes is $n_{y}$ :

$$
\left[\begin{array}{ccc}
P(\tilde{Y}=1 \mid Y=1) & \ldots & P\left(\tilde{Y}=n_{y} \mid Y=1\right) \\
\vdots & \ddots & \vdots \\
P\left(\tilde{Y}=1 \mid Y=n_{y}\right) & & P\left(\tilde{Y}=n_{y} \mid Y=n_{y}\right)
\end{array}\right]
$$

Note that all rows of the matrix in Eq. (2.6) are probability vectors that sum to unity. The error rate specific to each label is denoted as $p_{e}(y)=P(E=1 \mid Y=$ $y)$.

- Label Noise Not at Random (NNAR): This is the most general and realistic class of noise. This type occurs when the noise, E, could depend on either the true class label $Y$ and also on the values of the data, $X$. For example, there could be more errors in the labels of data instances near the classification boundary. Samples may be more likely to be mislabelled when they are similar to instances of another class, as illustrated in [70] where empirical evidence is given that more difficult samples in a text entailment data-set are labelled randomly. Here, the expected probability of error can be defined as in Eq. (2.7):

$$
p_{e}=\sum_{y \in Y} P(Y=y) \int_{x \in X} P(X=x \mid Y=y) P(E=1 \mid X=x, Y=y) d x .
$$

This type of noise can also be described for a specific region of values in the data using $p_{e}(x, y)=P(E=1 \mid X=x, Y=y)$. A recent study compared different methods for dealing with noise using new NNAR methods that simulated more realistic noise, i.e., when the noise occurred near the class boundaries [36]. The methods estimated which are the class borderline labels to be disturbed using two different criterion: the first method was based on the ratio of intra-class to inter-class nearest neighbor distance, and the second method was based on the distance between the examples and the decision border induced by a radial kernel [35]. 
This taxonomy works well to describe the types of noise in binary classification. However, in non-standard classification problems, additional dependencies may arise. These are not discussed in detail in the literature [87]. This thesis focuses on binary and multi-class classification, and there are some additional considerations required in studying noise in multi-class classification. Even state-of-the-art methods for dealing with binary class noise present considerable variation in performance when considering different multi-class noise patterns, discussed in the following section.

\section{Multi-class Label Noise}

In multi-class classification, each data point (text document) is assigned to a single class in the set $C=\left\{c_{1}, \ldots, c_{m}\right\}$, where $m>2$ is the number of classes. Class noise occurs when a true label is replaced by another label in $Y$. The traditional statistical taxonomy of label noise considers three categories, described in the previous section, and are still applicable in the case of multi-class noise. Besides those traditional types of noise, there are some additional specifics about the distributions to be considered for the multi-class scenario. In a recent study [87], the follow three types of distributions were studied, where in all the cases described below, the probability of a label being the true label is $1-p_{i}$ for true label class $i$.

- Uniform Distribution: The observed noise is evenly distributed among the classes. When a label is mis-labelled, it is equally likely to be any of the other incorrect labels. The probabilities for each true label $i$ to be an alternative label $j$ are $\gamma_{i j}=\frac{p_{i}}{m-1}$, where $p_{i}$ is the probability of error associated with that true label class, and $m$ is the total number of possible classes, and $i \neq j$.

- Natural Distribution: The noise distribution is proportional to the natural distribution of the remaining classes. This type of noise could be generated by a Gaussian random noise generator in a sensor. The probabilities for each true label $i$ to be mislabeled as $j$ (with $i \neq j$ ) is $\gamma_{i j}=\frac{p_{i} \pi_{j}}{1-\pi_{i}}$, where $p_{i}$ is the probability of error associated with that true label class, $\pi_{i}$ and $\pi_{j}$ are the class distributions, and $m$ is the total number of possible classes. 
- Default Class: Whenever a noise label occurs, it is incorrectly assigned to the same class, regardless of what the true class was. This may occur when a default label like "other" is assigned, when a true class label cannot be determined. If $d$ refers to the default error label class, the label probabilities would then be $\gamma_{i j}=p_{i}$ if $j=d$, and $\gamma_{i j}=0$ if $j \neq d$, for $j \neq i$.

- Blockwise: This occurs when there exists correlations among the noise patterns of some classes. An example of this would be pairwise errors, where two classes have their true class labels mislabelled. For example, in text sentiment classification, it is likely that two similar types of sentiment, like "anger" and "frustration", are more likely to have noisy mislabels between them. There are also cases where there may be more than two classes involved in this, and the noise patterns may be different for each, e.g., NAR vs NCAR. The probability transition matrix with two arbitrarily chosen blocks would be as follows:

$$
\gamma=\left(\begin{array}{ccccc}
1-p_{1} & p_{1} & \ldots & 0 & 0 \\
p_{2} & 1-p_{2} & \ldots & 0 & 0 \\
\vdots & \ddots & \ddots & \ddots & \ddots \\
0 & 0 & \ldots & 1-p_{n-1} & p_{n-1} \\
0 & 0 & \ldots & p_{n} & 1-p_{n}
\end{array}\right)
$$

These types of noise were studied in simulation studies, and compared when it concerned how they affected the performance of the classifiers. The findings were that uniform and natural distributions had a similar effect on the classifier's performance, except when the class balance became skewed, in which case the natural noise distribution was more harmful. They were also tested with two recent, state-of-the-art approaches to treat noise $[65,99]$. Both these methods performed well for uniform noise, but class imbalance, and the other distribution types, reduced the performance. NNAR-type noise caused problems, since some class distributions were likely to be differently influenced. For example, different class boundaries would cause class noise in different regions in the feature space, resulting in different blockwise interactions.

One method to deal with noise in multi-class labels, is to extend methods that were originally developed only for binary class. This can be done using data transformation 
approaches for schemes such as One-Versus-One (OVO) or One-Versus-All (OVA), that allow treating multi-class classification as multiple binary classifications. This approach has been taken in existing studies, however, by focusing only on the NCAR type of noise. It has been found that noise in minority classes is more harmful than in majority classes [115]. It is still unclear what learning effects multi-class noise can cause under highly imbalanced class distributions, and what methods can be used to handle it. Another effect that could arise in the multi-class situation, is that noise patterns can change the observed class ratio, and this can actually increase or decrease the presence of class-imbalances. Possible ways to handle noise in class imbalanced problems include cost-sensitive noise handling [124, 126], attributing and the development of class ratio aware filtering approaches [100].

\section{Effects of Label Noise}

The main objective of a classifier is to achieve a good performance on the predictions in the test set. One major effect that noisy labels can have is to decrease this prediction performance. Noisy labels can also negatively impact classification in other ways, including running time, size of the model and interpretability of the classifier built. These issues will be discussed in detail in the following section.

The main issue is the decrease in the classification accuracy. This decrease in performance due to noisy labels has been studied extensively, and it has been proven theoretically in several simple models. Some models that have been proven to have a performance decrease in the presence of label noise are linear classifiers [11, 40, 57, 72], quadratic classifiers [58] or kNN classifiers [82, 119]. For more complex models, there have been many studies to show such a decrease in prediction performance empirically for many models including Decision Trees, SVMs and Boosting (e.g. AdaBoost) $[26,47,79,88]$.

Noisy class labels can also have a negative effect on the classification model by increasing the number of required training instances [3, 5], and increasing the model complexity, that could, in turn, result in over-fitting, particularly in SVMs and Decision Trees [14, 88]. It can also negatively affect tasks upstream of the classification model, including feature selection $[28,38,107]$. Finally, noise in labels of the test 
data can affect model validation, since it could also cause inaccurate calculation of the performance measure $[20,60]$.

\subsubsection{Methods to Handle Noisy Labels in Classification}

\section{Robust Models}

Models robust to label noise are often referred to as "robust learners" [7]. Being "robust" to label noise, means that these models still work well in the presence of label noise without any particular pre-processing or modelling of the incoming label noise. However, if the level of noise is high, even these robust models will have challenges. More formally, the expected loss of a classifier under a given loss function is defined to be "label noise-robust" if the probability of mis-classification of the inferred models is identical, with or without the presence of class label noise [29].

There are some loss functions that have been theoretically proven to be robust in the presence of uniform (NCAR) label noise. These loss functions include the $0-1$ loss, which gives a cost of unity in the case of error of predicting a class label and is zero otherwise, and the least squares loss [68, 113]. Other, more practical, and more commonly-used loss functions have been shown to not be robust to label noise, including exponential loss (used in Adaboost), log loss (used in Logistic Regression), and hinge loss (used in SVMs).

There are many existing theoretical and empirical studies analysing which models are robust to label noise. Theoretical studies have shown that a large majority of ML algorithms are not completely robust to label noise [68]. Models and methods that prevent over-fitting have been shown to improve performance in the presence of noisy labels [68]. Techniques to avoid over-fitting, such as regularization can be used to partially handle label noise [112], even if the label noise may still reduce the accuracy of the classifier [112]. From empirical studies, even robust models have been shown to be affected by label noise except in overly simplified cases, and it has been shown that some models perform better in the presence of label noise than others $[27,53,102]$. The following list discuses some methods that are known to be robust to label noise.

- Ensemble Models: Ensemble models perform reasonably well in the presence of 
label noise. Bagging achieves better robustness than Boosting [26], because the diversity of the base classifiers is improved in Bagging, whereas the accuracy of the base classifiers in Boosting, such as in AdaBoost, is severely reduced. In comparing Boosting methods, it has been shown that there are several Boosting methods that are more robust than AdaBoost, including LogitBoost, and BrownBoost [91, 92, 93]. In a study comparing eleven classifiers on imbalanced data with asymmetric label noise, the ensemble model Random Forest was shown to be the most robust [1].

- Decision Trees: It is well known that Decision Trees can be prone to over-fitting and are therefore also quite highly affected by label noise. However, Decision Tree models, can be made more robust by using pruning strategies and setting the node splitting criterion to reduce over-fitting [7].

- LA: The only method that has been shown to achieve complete label noise robustness is one that uses LA, that directly optimizes the $0-1$ loss function. This has been shown in a previous study, using a team of continuous-action LA [102]. The mechanism used is as follows:

1. A probability distribution is defined on the weights of a linear classifier.

2. The weights are repetitively drawn from the distribution to classify training samples.

3. The 0-1 losses for the training samples are used at each iteration as a reinforcement to adjust the distribution around the weights.

The result of this is that the system was capable of converging to a class separating hyperplane.

Robustness of models in the presence of label noise with multi-class classification is more complex to analyse, both theoretically and empirically. To bypass this issue, it has been shown that one can use one-vs-one decomposition of multi-class problems can be used. This method of breaking down the situation into binary classification has also been shown to actually improve the robustness, which could be due to the 
distribution of the noisy examples, increasing of the separability of the classes, and collecting information from different classifiers [99, 100].

\section{Label-Noise Tolerant Learning Models}

The techniques described in this section are called noise-tolerant, because they can deal with label noise by modelling it. It is possible to design models that will take label noise into account by learning a model for the label noise, and by doing this simultaneously along with the classifier. This is a particularly good approach when there is knowledge about the type of label noise present. Another approach is to modify the learning algorithm in order to reduce the influence of label noise, for example by embedding data cleansing methods into the learning algorithm. The two main types of noise-tolerant methods to be discussed in the literature are probabilistic methods and model-based methods.

- Probabilistic Methods: Probabilistic methods include Bayesian and Frequentist methods, as well as clustering, and methods based on belief functions. These methods for detecting label noise require making assumptions about the distribution of the noise $[32,49,110]$.

- Bayesian Approaches: Several works claim that prior information is necessary to deal with label noise. In particular, some studies use Bayesian priors on the mislabelling probabilities [19, 50]. The Bayesian approach considers that all the unknown parameters have a probability distribution that reflects the uncertainty in their values, and that a prior knowledge about these unknown parameters can be formally included. The Bayesian approach is a generalization of constraints on the parameters values, and has been used to set priors on model parameters and mislabelling probabilities $[31,50]$. Popular choices for Bayesian priors for label noise are Beta priors [31, 50] and Dirichlet priors [98]. A major advantage of using priors is the ability to include existing information in the learning process. However, the results obtained depend on the quality of the prior distribution chosen and could potentially have negative affects as well [59]. 
- Frequentist Approaches: A simple frequentist approach involves using mixture models, to treat label noise in a similar way to outlier detection [86]. Probabilistic modelling of the label noise can provide an estimate of the level of the noise in addition to improving the classification. Methods have been developed to deal with NCAR and NAR label noise [64]. These method have been used in Logistic Regression and NNs [61, 90].

- Clustering: These methods assume that the labels are linked to the features of the instances of the data. Clustering of the data instances is performed first in an unsupervised way. This can then be used to adjust classifiers in the presence of noisy labels, for example, by computing a confidence on the labels based on the cluster that each data point is in [94].

- Belief Functions: Belief functions allow distinguishing subjective uncertainty from objective uncertainty. They can be given directly by the expert annotator, or be inferred with a kNN rule [111]. Each possible class is characterised by a belief mass, which is the amount of evidence which supports the class. Belief functions can be used to modify standard ML methods such as the kNN classifiers, NNs, Decision Trees, mixture models or Boosting [28, 29, 30].

- Model-Based Methods: Strategies have been developed to obtain label noisetolerant variants of many algorithms commonly-used in text classification, including SVMs, NNs and Decision Trees [30]. Common approaches include modifying the losses, and using methods that prevent over-fitting. Numerous publications also focus on label improving noise-tolerance for Boosting algorithms such as AdaBoost, since these methods are known to be very sensitive to label noise [55].

In summary, in these approaches, the complete model of the training data consists of a label noise model and a classification model. Both of these phases are used during training, but only the classification model is is used for predictions. Dropping the label noise part of the model is only possible when the label noise is explicitly modelled, as in the probabilistic approaches. For the other approaches, the learning process of the 
classification model is supposed to be robust or tolerant to label noise and to produce a good classification model. The main problem with the approaches described in this section is that they increase the complexity of the learning algorithms, and can lead to over-fitting, because of the additional parameters of the training data model used to model the noise.

\section{Data Cleaning}

Data cleaning is a way to reduce the effects of noisy labels, and can be used independently of the classifier model, before training it. This can be time consuming, and is a good option when the datasets are small. There are many approaches to achieve this, and it has been shown that using these methods to identify and remove noisy labels can improve the performance of the classifiers. Identifying label noise can be done in a similar way to outlier detection and anomaly detection [6, 17, 42]. Mislabelled instances can also be identified as instances that causes relatively large increase in model complexity [33, 34], or a relatively large influence on the learning $[67,123]$. Another major approach is known as noise filtering. This is a good strategy to obtain better results in the presence of even low amounts of noise [87].

One can also use the predictions from the model to identify mislabelled data, and filter them out iteratively. Inaccurate predictions can be iteratively removed throughout training the model, as a simple approach to filter out possible mislabelled instances $[34,45,52]$. An alternative method to filter out possible incorrectly labelled instances is called "Voting". Voting filtering has been tested in many previous studies $[14,15,34,52,116]$. It works with ensemble methods, and removes instances based on the votes of the learners in the ensemble.

Model-specific cleaning methods have also been reported, and these use the properties of the classifier model to identify possibly-mislabelled instances. For example, AdaBoost tends to give large weights to mislabelled instances, and so this can actually be used as a way to detect these mislabelled data [116]. kNN-based methods can be used to remove instances that do not cause other instances to be further misclassified $[22,37,118,119]$. There are other additional approaches related to data cleaning, that involve semi-supervised learning. The idea behind these is to achieve a partial 
data-cleaning, removing only the labels, but leaving the data itself to be re-labelled with a semi-supervised method [43].

\subsubsection{Experimental Considerations}

This section will discuss how experiments have been performed in the literature to study label noise. A large majority of the studies on noisy labels relies on simulating noise into existing given labels. This is due to the fact that there are very few real world data-sets that have had their real mislabelled instances corrected $[20,46,63,67$, 96]. We will discuss the methods of generating label noise, as well as the mechanisms regarding how one can compare algorithms in the presence of noise, quantitatively.

\section{Label Noise Generation}

To study label noise, the noise is most-commonly artificially simulated, because there are very few existing data-sets where the noise has been corrected. Another benefit of simulating the noise, is that it can be simulated in particular ways so as to isolate the testing of various types of noise, and to characterise the performance of models in various scenarios. There are various ways to artificially introduce noise into the existing labels. Indeed, noise can be introduced for each of the methods described previously in Section 2.5.1.

- NCAR: This type of noise is introduced by picking instances at random and flipping their label;

- NAR: This type of noise can be generated by using a similar approach to NCAR, but the flipping must be different for each class. There are several strategies for asymmetric label noise generation $[27,41,53,79,124,125]$. One commonlyused type of noise is called "pairwise", where labels are flipped for only a single class. For example, in binary classification, it could be that there is more noise in the minority class, and so the pairwise noise simulation would be to flip the labels of the minority class in a certain percentage of the instances. 
- NNAR: This type of noise is much less studied, although there are some existing methods [102] where noise is injected in higher proportions depending on the feature values. More recent methods of injecting noise attempt to simulate realistic scenarios, where the noise is injected close to the class boundaries [36].

\section{Performance Measures for Algorithms}

Most experiments assess the efficiency of methods to take care of label noise in terms of accuracy [30], since a decrease in accuracy is one of the main consequences of label noise, as discussed in Section 2.5.1.

Another common criterion is the model complexity [30]. For example, the number of nodes in the Decision Tree is a valid criterion, because, as discussed in Section 2.5.1, some algorithms tend to over-fit in the presence of label noise. In the case of data cleansing methods, filter precision can also be measured by assessing whether the removed instances actually correspond to mislabelled instances.

The robustness of a classifier can be quantified by the "Relative Loss of Accuracy" (RLA) as described in Eq. (2.9), which is used to measure the percentage of the change in the classifier's accuracy at certain pre-set noise levels [28].

$$
R L A_{x \%}=\frac{A_{c c 0 \%}-A_{c c x} \%}{A_{C C 0 \%}} .
$$

In Eq. (2.9), $R L A_{x \%}$ is the RLA at noise level $x \%$, and thus $A_{c c 0} \%$ is the test accuracy in the presence of no added noise, and, $A_{c c x} \%$ is the test accuracy with the added label noise.

\subsection{Conclusion}

This chapter surveyed the fields of research that focus on classification for the application of text data. This survey also reviewed the challenge of text classification in the presence of noisy labels in the training data, as is commonly the case in human generated training labels. Finally this chapter reviewed the field of LA. The thread that ties these areas is the application of LA for the purpose of text classification. Since 
LA have the ability to operate in noisy environments, this is a worthwhile approach to combat this challenge present in text classification.

The survey has illustrated that there is a wide range of approaches available for text classification for each step of the pipeline, starting from the raw input text data, through a series of steps to convert it into a numerical vector representation, and finally to achieve the final trained classifier and output of the predicted labels for the test data. There is a wide variety to the complexity of these approaches, from simple bag-of-words, to state-of-the-art transformer models. This survey has also shown that noise in training labels is a widely studied problem in machine learning research. Numerous approaches exist to detect it and to simulate it for the purpose of experiments. However there are currently no systematic studies that test the effects of various types of injected noise for the application of text classification, so this remains to be studied.

Finally the last section of this chapter reviews the field of LA. LA are capable of learning in noisy environments and have been studied for many decades. The recent development of the Tsetlin Machine for text classification has brought the field of LA into relevance for text classification. To review this field, many classes of LA were described in detail. Future work remains to investigate the performance of the novel Tsetlin Machine in various classification experiments, including in the setting of noisy training training labels. 


\section{3 \\ Classification with Injected Label Noise}

\section{$3.1 \quad$ Introduction}

The previous chapter, that surveyed the field of NLP, can be perceived to be twopronged. The first direction involved the modelling of the documents, i.e., tackling the task of transforming text-based data into numerically assigned vectors. As mentioned there, a host of such models are available, such as TF-IDF, count vectors, and pre-trained GloVe vectors. The second direction, which we addressed, dealt with classification algorithms, which specifically attempted to classify the documents into their respective classes. The task that we will embark on in this chapter is to compare text classification algorithms and text representations for classification. We should also mention that the study would be incomplete if we did not consider the influence of noisy labels. Such a scarlet thread will go throughout the entire section. In other words, we will consider algorithms and models where the labels are garbled 
with noise. In particular, we shall deal with class-dependent and class-independent random noise, as discussed in Chapter 2.

Before we proceed, we have to accept that it is not possible to implement all the available/reported classification and representational methods. As mentioned in Chapter 2, there are a host of traditional schemes, such as kNN, Decision Tree, Naive Bayes, etc. Although these are not predominantly used in text classification, our task here is to compare a diverse set of approaches and see how each of these are affected by different types of noise in the class labels.

In addition to comparing text representation methods and traditional classification algorithms, we will also discuss recently-developed methods which each have their own specific data representation. There are a few such methods reported in the literature, and we concentrate on the two which we believe are the most interesting to compare in these experiments. The first of these is the BERT algorithm [25], introduced in Chapter 2, which has its own document representation strategy by fine-tuning the $[C L S]$ token for the text classification task. The second is the LA scheme, which uses the Tsetlin Machine. Our aim here is to study how these two methods function within their own data representational method, and compare their performance in the presence of different types of injected label noise.

In the first section of this chapter, we will discuss the experimental set up used to study these classification methods. This section will describe the dataset used, and the full experimental pipeline that the data runs through to output the final classification performance metrics. In the second section, we describe, in detail, the artificial noise injection methods. These are the methods used to simulate noise in the labels of the labelled dataset. Finally, in the last section, there will be an analysis and discussion of the results for our two main investigations, namely, 1) the effects of the noise types on the performance of the two recently-developed classification methods and the traditional classification methods, and 2) the effects of the noise types on the performance of the classification schemes and the document representations being compared. 


\subsection{Classification Experiment Setup}

This section will provide details on the experimental setup used to test several classifiers for text classification with noisy labels, with noise simulated by the various approaches described, in detail, in the following section.

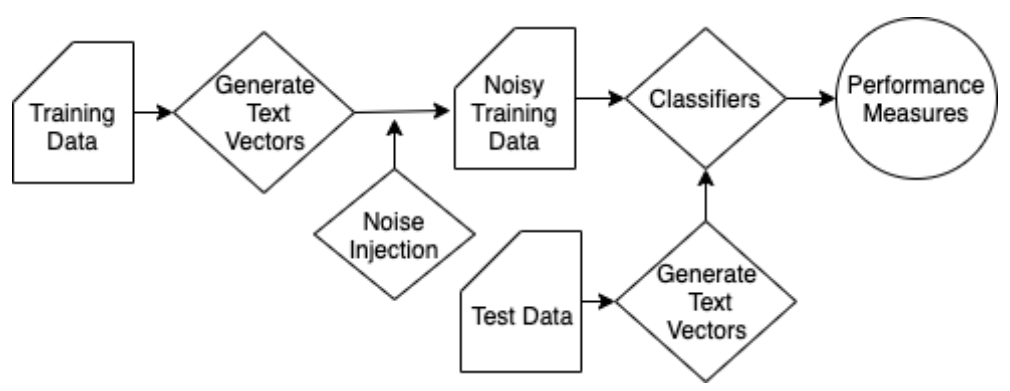

Figure 3.1: Overview of experiment methodology

The diagram shown in Figure 3.1, provides an overview of the experimental set up. Each of the main components of this experimental pipeline will be explained in further detail, starting with an overview and a characterization of the dataset used.

\subsubsection{Text Classification Data Set}

The IMDb sentiment classification dataset [66] was used for these experiments, and it is a widely-used benchmark dataset for testing classifiers in NLP. It is commonly used for binary sentiment classification, using the text reviews to predict a positive or negative sentiment. The IMDb data set has 50,000 movie reviews, with a fairly even number of reviews from each class, and with no more than 30 reviews per movie. Neutral reviews from IMDb are not included in the data set. The labels in this dataset are given by the users who wrote the review, and so these labels should be as close to the ground truth as possible, since it avoids the possibility of errors by subsequent annotators reviewing and interpreting someone else's appraisal to provide a label. These labels can, therefore, be used as a good basis for training the classifiers with a noise level of 0 . This will form the starting point for simulating noisy labels in experiments, which will be done by injecting noise into these ground truth labels. 


\subsubsection{Generating Text Vector Representations}

The first step in the experimental pipeline is that of generating the text vector representations of each of the text reviews in the dataset. There are several methods for doing this. In our experiments, we use three main text vector representations that invoke the traditional classifiers. These representations are:

- Count vectors

- Term frequency inverse document frequency (TF-IDF)

- GloVe pre-trained vectors

For two of the classifiers, the BERT and the LA approach, certain alternative vectorization approaches were required. For the BERT classifier, the standard BERT pre-processing approaches were applied, as described in Chapter 2. For the LA ap-

proach, we first used the $\chi^{2} \mathrm{k}$-best feature selection, and then the text was processed into binary vectors, using the method described in the paper by Berge et al. [56], and explained in greater detail in Chapter 4.

\subsubsection{Noise Injection}

The noise injection methods used in this experiment is described in detail in Section 3.3. Each of these noise injection methods was used to first generate an ordered list of label indices to be flipped. Then, based on the specified level of noise, $p$, a certain percentage of the labels in the training data were flipped, in that order. The noise levels used were $p=0.3$ and $p=0.15$ for all the classifiers. For random noise, and class conditional noise, the noisy label orders were randomly generated ten times. This process yielded a matrix of noisy labels, with a single column for each noise type. This matrix of labels was then used to train each classifier in the next phase.

These injection methods can also be extended to study noise for multi-class classification, as described in Section 2.5.1, though the following experiments will focus on binary classification. 


\subsubsection{Training Classifiers}

The main types of classifiers being investigated in this experiment were:

1. Several traditional classifiers implemented with scikit-learn that have diverse types of decision boundaries.

2. A state-of-the-art classifier for text classification (BERT)

3. A novel classification approach based on LA

These classifiers were described in more detail in Chapter 2, and we provide here only some implementation-specific details.

First, the python library scikit-learn [83] was used to implement the following classifiers:

- Logistic Regression

- Naive Bayes

- AdaBoost

- Decision Tree

- $\mathrm{kNN}$

Each of these classifiers was trained with various hyper-parameters, tuned using a grid search, and trained on several different text representations. More details on this procedure is shown in Figure 3.2.

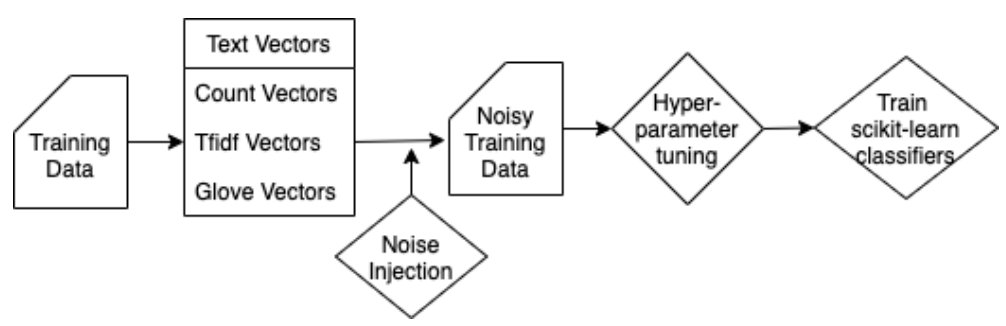

Figure 3.2: Scikit-learn classifier training procedure 
Each classifier yielded output results for each input text representation, and so this portion of the experiment generated a total of 18 different sets of predictions to be compared for each noise type, given that the three different input text representations were each tested with 6 classifiers.

The next type of classifier tested, called BERT [25], is currently a state-of-the-art performing method in NLP, and has been used on a wide range of tasks. The implementation used was from a python library called Transformers [120]. This method involves its own method of pre-processing, and we followed such a standard procedure, where the hyper-parameters were chosen based on the recommended values in the literature. Finally for the LA classifiers, the implementation was from the code accompanying the paper by Berge et al. [10].

\subsection{Noise Injection Methods}

Various noise injection methods were used in the experiments to simulate different types of noise in the training labels. This section will provide the specific details on each noise injection method, including novel noise injection methods. This section will also analyse how well these types of noise can be identified by noise detection methods.

\subsubsection{Noise Types}

\section{Random Noise and Class-Conditional Noise}

In the early days of research on learning from noisy labels, Angluin and Laird first proposed the random classification noise (RCN) model, where each label was flipped independently with some probability $p \in[0,1 / 2)$. This is the most commonly-studied type of noise, used in testing noise robustness of classification methods. It simulates noise as if every label has an equal chance of being corrupted, regardless of the characteristics and positions of the examples. Random noise was simulated by randomly choosing $N$ labels that were to be corrupted, specified by the set noise level, $p$. The algorithm used is shown in Algorithm 1. 


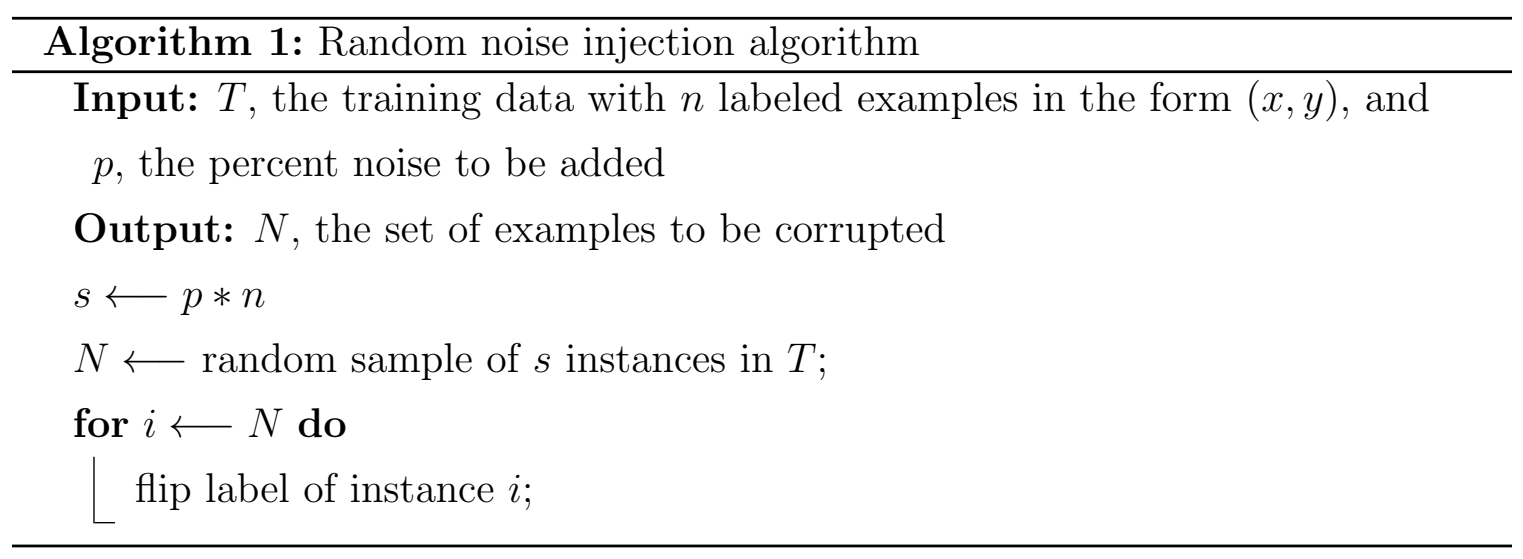

In Class-Conditional label Noise $(\mathrm{CCN})$, the noise rates depended on the original class labels of each instance. For this type of noise, the same algorithm was used as the algorithm for RCN, Algorithm 1, except it was run on the instances of each class separately, with different values for $p$ for each class.

\section{Border Noise}

The border noise methods used in these experiments are based on the paper by Garcia et al. [35], which proposed novel noise injection methods to corrupt labels that are closest to the class boundary, since such a scheme would, possibly, be a more realistic way to simulate noise that may actually occurs in real datasets.

In order to determine which instances are closest to the class boundary, we used several methods. All the border noise injection methods followed the general format of the algorithm shown in Algorithm 2. The issue, in question, is that of determining how to compute the distance of each instance to the class boundary. Given a labelled dataset of real-world data, there is no pre-specified class boundary, and so this boundary must be determined by some calculation. Two methods considered in the previous study by Garcia et al. are neighbour-wise boundaries, and non-linear class boundaries.

The neighbour-wise method computes a value for noise in a manner similar to a $\mathrm{kNN}$ classifier. This noise injection method uses the ratio of the intra-class distances 
and inter-class distances to analyze the spread of the examples from distinct classes, and to determine the borderline examples, where the intra-class distance is the distance between examples from same class, while the inter-class distance is the distance between examples from distinct classes. The value computed relies on these distances and the labels of the closest points to each instance. The distance metric used with this method in the original paper was the Euclidean distance. Since this distance measure is not widely used for distances between document vectors, the metric that was adapted for the purposes of text classification was by using the cosine distance, which is a more commonly-used distance measure in the text domain.

The non-linear class boundary noise value is determined by first training a SVM with a Radial Basis (RBF) kernel. Once this classifier was trained, a decision function was obtained, $f(x)$. This function was then used to compute the value of noise to give each instance in the training set, as $|f(x)|$. This method was extended to be more applicable to text classification. In text classification, class boundaries are predominantly linear, and so a linear SVM classifier was also used.

Therefore, four different class boundary noise injection methods were used, each following the same algorithm, Algorithm 2, with varying methods of computing the noise value for each instance. The four noise value calculations are shown in the following equations in Table 3.1 . 


\begin{tabular}{|c|c|}
\hline $\begin{array}{l}\text { Boundary } \\
\text { Noise Type }\end{array}$ & Noise Value Equation \\
\hline $\begin{array}{l}\text { Neighbour-wise } \\
{[35]}\end{array}$ & $\begin{array}{l}\frac{d\left(x_{i}, N N\left(x_{i}\right) \in y_{i}\right.}{d\left(x_{i}, N N\left(x_{i}\right) \notin y_{i}\right.} \text {, where } N N \text { are the nearest neighbours of the } \\
\text { point } x_{i} \text {, and } y_{i} \text { is the class label of } x_{i} \text {. The distance measure, } \\
d \text {, is the Euclidean distance. }\end{array}$ \\
\hline $\begin{array}{l}\text { Text Neighbour- } \\
\text { wise }\end{array}$ & $\begin{array}{l}\frac{d\left(x_{i}, N N\left(x_{i}\right) \in y_{i}\right.}{d\left(x_{i}, N N\left(x_{i}\right) \notin y_{i}\right.} \text {, where } N N \text { are the nearest neighbours of the } \\
\text { point } x_{i} \text {, and } y_{i} \text { is the class label of } x_{i} \text {. The distance measure, } \\
d \text {, is the cosine distance. }\end{array}$ \\
\hline Non-linear [35] & $\begin{array}{l}|f(x)| \text {, based on the decision function } f \text { obtained from a } \\
\text { trained RBF SVM. }\end{array}$ \\
\hline Linear & $\begin{array}{l}|f(x)| \text {, based on the decision function } f \text { obtained from a } \\
\text { trained linear SVM. }\end{array}$ \\
\hline
\end{tabular}

Table 3.1: Noise value equations

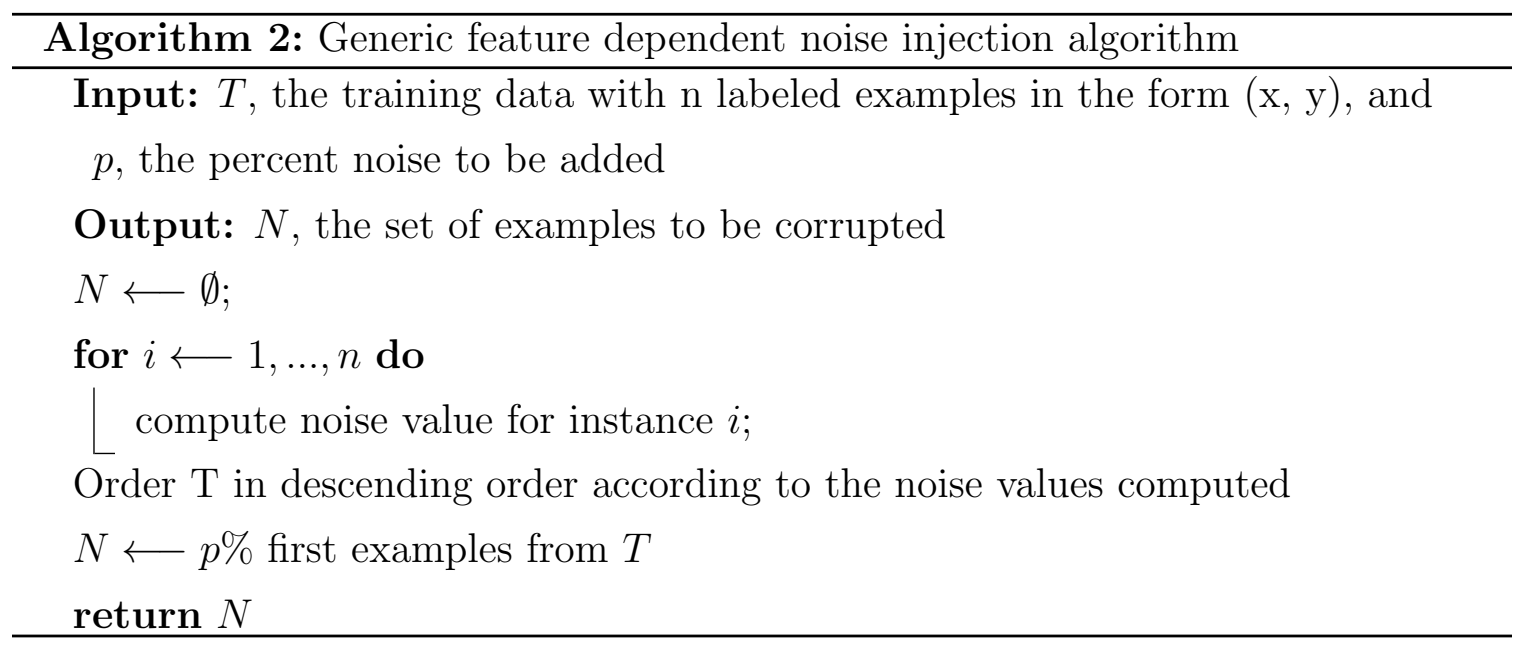


The rationale behind these border noise injection methods was to test cases of more realistic types of noise. There is more likely to be mistake in a real dataset's annotations in the instances that are more ambiguous, and closest to the middle of the two classes. This makes sense in the case of text classification, since it is likely that in a large corpus to be annotated, there will be many examples where it is unclear to the annotators which class the document should belong to. This is because of the nature of text classification problems, where the tasks are often very subjective and hard to define, even for humans. So, it is more likely that the annotators may make mistakes in choosing which label is to be given to these training examples.

In annotating for text classification, it is also possible that annotators may simply make mistakes due to a variety of other reasons, such as misreading the text, or in selecting the annotation in the annotation software. Another possible scenario, is that there will be some documents that are outliers in the dataset, which are quite different from most other documents. This could also cause some confusion for the annotators. In such cases, these documents would lie quite far away from the class-boundaries, due to the nature of text representation problem being high-dimensional, and the fact that these documents would be quite different from most of the other documents in the dataset. In order to study this opposite extreme of border noise, the reverse metrics of those described above were also used, to simulate noise in instances furthest away from the class boundary. Therefore, in total, 8 border noise injection methods were included in the experiment. Throughout the rest of this document, these noise types will be referred to in an abbreviated form when needed. This abbreviated form is explained in Table 3.2 .

\section{Feature-dependent Noise}

In this thesis, we also studied feature dependent noise. Feature dependent noise,

previously studied by Manwani and Sastry [68], was the case when two features were randomly chosen, and the labels were corrupted proportional to chosen noise levels for the quadrant of the combination of these features. This method was adapted for use in text classification, where the features in question were the words in the documents. Instead of randomly choosing features, a set of terms was chosen based on a lexicon. 


\begin{tabular}{|c|c|}
\hline Border Noise Type & Abbreviated Code \\
\hline \hline Neighbour-wise & border \\
\hline Reversed Neighbour-wise & rev-border \\
\hline Text Neighbour-wise & border-text \\
\hline Reversed Text Neighbour-wise & rev border text \\
\hline Non-linear & border nonlin \\
\hline Reversed Non-linear & rev border nonlin \\
\hline Linear & border lin \\
\hline Reversed Linear & rev border lin \\
\hline
\end{tabular}

Table 3.2: Noise type abbreviations

This approach can be used to simulate noise in situations where certain terms are more likely to cause different responses by the annotators. This could include terms that may confuse the annotators (typos, foreign languages, complex terminology), terms that may provoke different reactions in different people (terms associated with biases), and also even strongly emotional terms or sarcasm, since sarcasm can often be misinterpreted in text. The chosen lexicon used for these experiments consisted of bias terms developed by Recasens et al. [95]. The noise value was computed for each instance $x$ using the following equation:

$$
p_{x}=\frac{\sum_{i}^{n} 1_{\left\{w_{i} \in L\right\}}}{n},
$$

where $p_{x}$ is the noise value for instance $x, L$ is the set of terms in the bias lexicon, $n$ is the number of tokens in the document $x$, and $w_{i}$ is each token. This equation computes the percentage of the document that was made up of terms that are in the bias lexicon. This value was computed for each document in the training set, and the noise was injected by Algorithm 2, with a chosen noise value, $p$.

\section{Document Related Noise}

Another aspect of text classification is related to the document itself, for example document length. Document length plays a significant role in text classification when trying to detect meaning in documents. For example, typically, the length of the document is considered to be independent of the document's topic, and this is why 
cosine distances are often used, i.e., to prevent relating documents based on the lengths of their vectors, and by allowing the system to focus only on their orientation. However, in practice, it is possible that longer documents may cause problems during the annotation process. Longer documents are actually more likely to contain a mix of topics, and the true class may be more unclear to the annotators. Also, it is possible that in some cases, annotators may not fully read the longer documents, such as in cases when they are being paid per annotation (such as mechanical turks). This could also result in a higher chance of error associated with longer document lengths.

In order to test a classifier's robustness to length-dependent noise, noise was injected to the labels of the longest documents in the training set, based on the number of tokens (i.e., words) in the document, once again as per Algorithm 2. This allowed us to study another uniquely-specific noise type for the application of text classification.

\subsubsection{Analysis of Noise Detection}

Given all these types of noise, the first question that arises is to determine how well each of these types of label noise can be identified by a noise detection method. In order to test this, a recent method, known as "confident learning" [80], was used to detect the noise injected by each of these methods. Confident learning is an approach for characterizing, identifying, and learning with noisy labels in datasets, based on the principles of pruning noisy data, counting to estimate noise, and ranking examples to train with confidence.

Confident learning was applied to assess how well it could be used to detect the noise injected by various methods. The performance of this method is shown in Figure 3.3 and in Table 3.3. The precision and recall scores are both shown, because in correcting noisy labels, it is important to realize that in some cases, it may incorrectly identify labels as being noisy, and in trying to correct these it could result in actually introducing more errors into the labels. This scenario would arise if the precision is low. Recall is also important to consider because, it would also be ideal to correct as many of the true noise labels as possible. With regards to precision, the linear boundary noise type was detected quite well by confident learning methods. Confident 
learning also demonstrated a rather high performance on random noise detection. The class-dependent noise and the non-linear border noise types were much more difficult to detect by using this method.

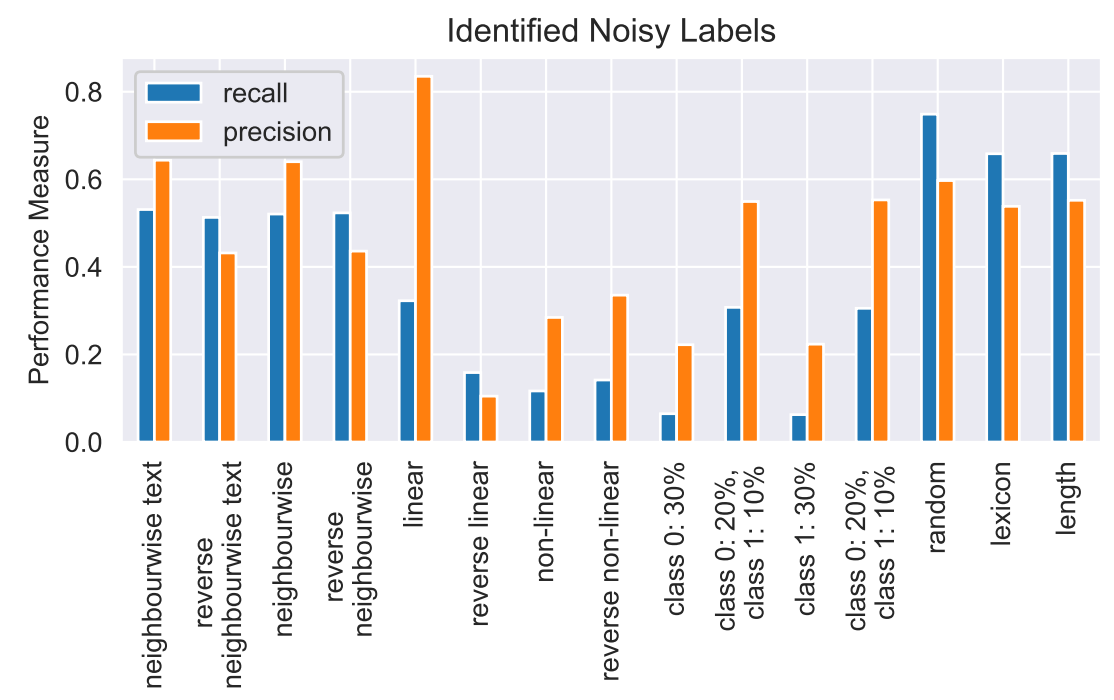

Figure 3.3: Identification of noisy labels with confident learning

Confident learning was also used to train a "learning with noisy labels" classifier, implemented by the python package "Cleanlab". The base classifier used was Logistic Regression, and it was compared with a simple version of Logistic Regression implemented by scikit-learn. As can be seen from Figure 3.4 and Figure 3.5, this method was not successful in improving the performance above the original results of a regular Logistic Regression in the presence of noisy labels. 


\begin{tabular}{|c|c|c|}
\hline & Recall & Precision \\
\hline \hline neighbourwise text & 0.53 & 0.64 \\
\hline reverse neighbourwise text & 0.51 & 0.43 \\
\hline neighbourwise & 0.52 & 0.64 \\
\hline reverse neighbourwise & 0.52 & 0.43 \\
\hline linear & 0.32 & 0.83 \\
\hline reverse linear & 0.15 & 0.10 \\
\hline non-linear & 0.11 & 0.28 \\
\hline reverse non-linear & 0.14 & 0.33 \\
\hline class 0: 30\% & 0.06 & 0.22 \\
\hline class 0: 20\%, class 1: 10\% & 0.30 & 0.54 \\
\hline class 1: 30\% & 0.06 & 0.22 \\
\hline class 0: 20\%, class 1: 10\% & 0.30 & 0.55 \\
\hline random & 0.74 & 0.59 \\
\hline lexicon & 0.65 & 0.53 \\
\hline length & 0.65 & 0.55 \\
\hline
\end{tabular}

Table 3.3: Precision and recall of Cleanlab with Logistic Regression on IMDb Dataset

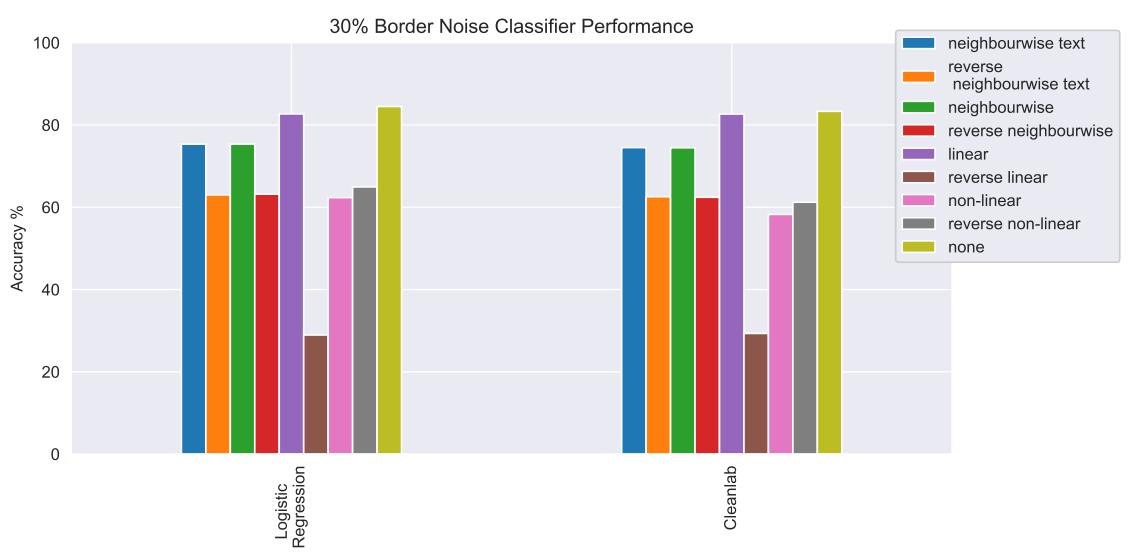

Figure 3.4: Cleanlab performance in classification with border noise 


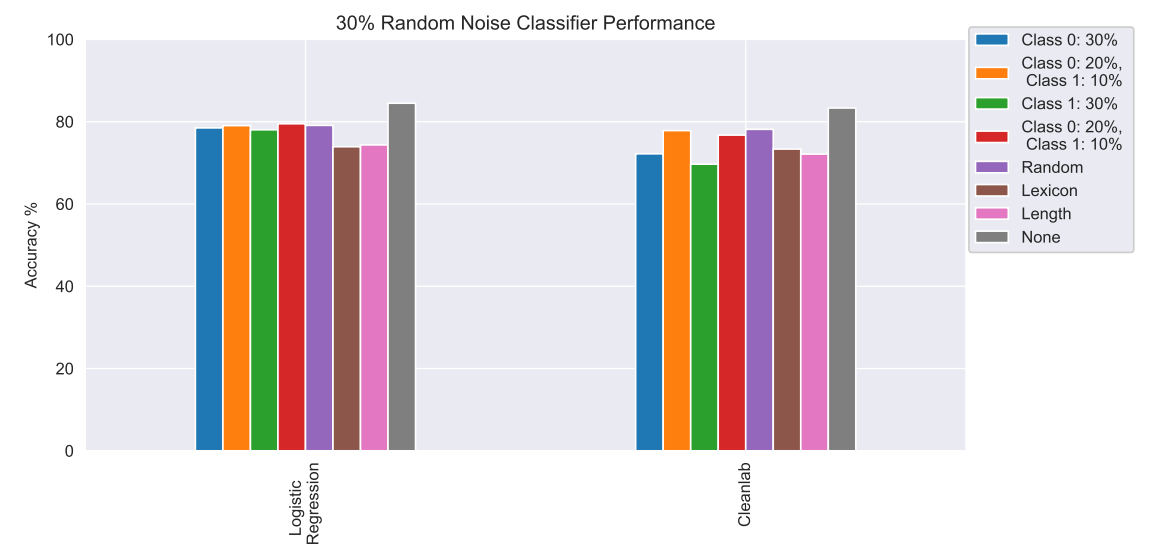

Figure 3.5: Cleanlab performance in classification with random noise

In conclusion, these results show that detecting noise present in labels for text classification may be effective in some cases, for example, when the noise is distributed randomly. However it may pose challenges when the noise is present in different patterns, such as in the case of non-linear border noise, or class-dependent noise. Also, the confident learning method used for classification was not seen to result in an improvement in the overall classification performance. Bearing these results in mind, we opted to run further experiments to determine the effects of these noise types on different families of classifiers, and these results are given in the following section.

\subsection{Analysis of Results}

The task that we will embark on in this section is to understand which classification algorithms and which text representations are better suited for classification in the presence of different types of label noise. We will study various classifiers and various text representations with different types of noise injected into the class labels, as described earlier.

In the first half of this section, we will focus on the traditional classification schemes, such as kNN, Decision Tree, Naive Bayes, etc. Although these are not 
currently used predominantly in text classification, our task here is to see if one can obtain an accuracy comparable to the state-of-the-art methods by invoking these schemes. Further, we would like to compare how each of these are affected by different types of noise in the class labels. These methods will each be tested with various text representation methods.

The second half of this section will concentrate on methods which each have their own specific data representation. There are a few such methods reported in the literature, and again we will concentrate on the two which we believe are the most promising. The first of these is the BERT algorithm [25], introduced in Chapter 2 , which has its own document representation strategy produced by fine-tuning the $[C L S]$ token for the text classification task. The second is the LA scheme, which uses the Tsetlin Machine. Our aim here is to study how these two methods function within their own data representational method, and in the presence of label noise.

\subsubsection{Effects of Document Representations}

This section will analyze the effects of representing the text with various representational methods, that were each described, in detail, in the literature review in Chapter 2. First, the text dataset was vectorized, and subsequently various characteristics of the dataset was assessed. Thereafter, each of these methods was tested for performance in classification with and without the injected label noise. This allowed a comparison of each of these representations for the standard task of classification. It also provided a comparison of the representations that were most affected by the injected label noise.

As previously described in Section 2.2, there are many ways of representing text in the form of a vector. The three ways which will be used for the following analysis are:

1. Counts: based on the counts of words in the document.

2. TF-IDF: based on the frequencies of words inversely related to the frequency across documents. 
3. Word-vectors: Pre-trained word vectors, aggregated into document vectors.

The pre-trained word-vectors chosen for this experiment were the well known and previously described "GloVe" vectors. These word vectors were combined into a document vector, by taking the mean of all the word vectors in the document. This can be considered as representing the document by the centroid of all the word vectors. These word vectors were initialized with weights from training on a large corpus to encode meaning of the words into the form of a vector.

\section{Data Characterization}

The following analysis will characterize the IMDb dataset, using various text vector representation methods to represent the text documents in a numerical space. Each of the three listed text representations were analysed, and characterized following the same methods as per the paper by Garcia et al. [35]. This paper inspired the design of these noise injection experiments, and so the dataset was also characterized in a similar way, in order to relate back to this original paper. The three main characteristics used were: the dimensionality ratio, imbalance ratio (IR) and the error ratio of a Linear Classifier $\left(L_{2}\right)$.

The dimensionality ratio indicates the data sparsity by considering a minimized set of uncorrelated features. In the original paper by Garcia et al. [35], Principal Component Analysis (PCA) was first applied to each dataset. In the context of text vector representations, Latent Semantic Analysis (LSA) was the more appropriate approach, since mean centering does not make sense in the context of word counts. The LSA was applied using the scikit-learn implementation. The components were extracted, and then the number of components necessary to represent $95 \%$ of the variability in the data, $d^{\prime}$, is calculated. Next, the ratio $\frac{d^{\prime}}{d}$ was calculated to indicate the sparsity, where $d$ is the number of original dimensions in the dataset. The IR measure divides the number of examples in the majority class by the number of examples in the minority class, which indicates whether class imbalance is present in the dataset, which we know was not present in this dataset. Finally, the error rate $\left(L_{2}\right)$ was calculated using the error rate of a linear classifier. A SVM classifier using 


\begin{tabular}{|c|c|c|c|}
\hline & TF-IDF & Word Counts & GloVe Vectors \\
\hline \hline Dimensionality Ratio & 0.7809 & 0.5339 & 0.6099 \\
\hline$L_{2}$ & 0.1036 & 0.1022 & 0.1549 \\
\hline IR & \multicolumn{3}{|c|}{0.503} \\
\hline
\end{tabular}

Table 3.4: IMDb data characterization

a linear Kernel was trained on the training set, and tested on the training set as well. The error rate (i.e., accuracy) indicated how linearly separable the training set was. Each of these metrics was computed on each of the five folds used in the cross validation experiments, and the means of each metric are shown in the Table 3.4

Comparing these results to the datasets characterized by Garcia et al. [35], these text representations were seen to be the mid-range in sparsity based on the dimensionality ratio, on the low end of error rates for linear separability (meaning that they were reasonably well separable), and there was an even class balance between the positive and negative classes. The counts and TF-IDF vectors were very similar in linear separability, and the count vectors were slightly more sparse than the TF-IDF. The reader should observe that the original dimension sizes of these vectors were specified in the vectorizers that produced the TF-IDF and the counts vector representations as a means of pre-processing, so as to remove the overly common words (stop words), or rare words. The original dimensionality of both of these vectors was 800 , and the GloVe vectors chosen were of dimension 300. These vectors were similar in sparsity as the other two methods, though it demonstrated a slightly reduced linear separability.

\section{Performance in Classification}

Each of three text representation methods was tested and compared based on classification performance with the Logistic Regression classifier under each type of injected label noise. It can be seen from the results in Figure 3.6 and Table 3.5, that across all noise types, each document vector's performance was quite similar. The GloVe word vectors performed slightly better in most cases, though the differences were quite small. The most notable difference in performance was seen in the reverse linear noise type, where the performance of the GloVe vectors was the strongest, and the 
TF-IDF vectors's performance was quite a bit lower.

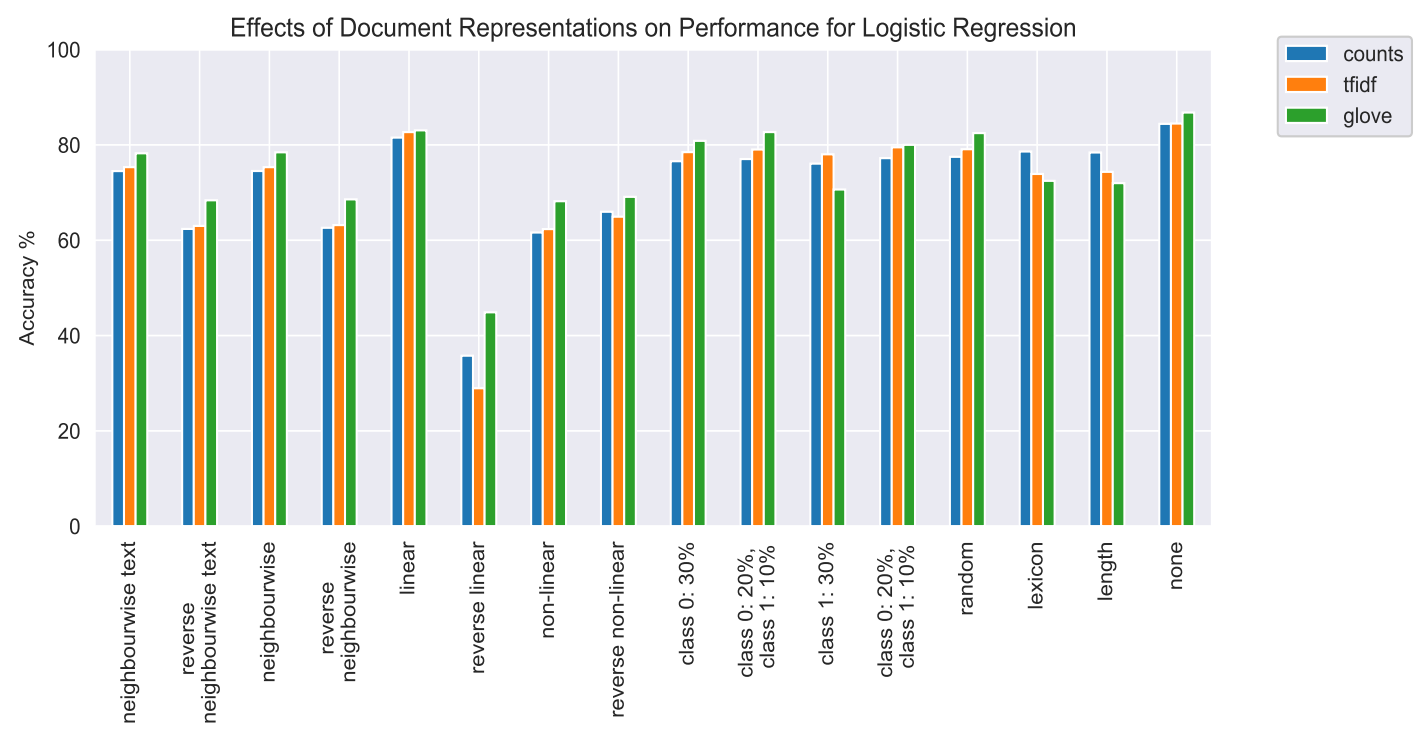

Figure 3.6: Performance of Logistic Regression classifier with 30\% noise

\subsubsection{Analysis of Results by Classifier Type}

This section will discuss the results of the three main classifiers being compared: Logistic Regression, BERT, and the Tsetlin Machine. These classifiers were each tested in the experimental set up described in the previous section, trained on the original labels as well as on the labels injected with the different types of noise. The results for each of these three main classifiers will be discussed in the following sections.

\section{Logistic Regression}

The Logistic Regression model is one of the most commonly used baseline models in NLP, since usually a simple linear boundary is all that is required for separation in high dimensional spaces. The performance of this model in these experiments was overall quite good, and comparable with the state-of-the-art BERT model in the case with no noise, as seen in Figure 3.8, and in Table A.1. Also, the results show that 


\begin{tabular}{|c|c|c|c|}
\hline & counts & TF-IDF & GloVe \\
\hline \hline neighbourwise text & 0.74 & 0.75 & 0.78 \\
\hline reverse neighbourwise text & 0.62 & 0.63 & 0.68 \\
\hline neighbourwise & 0.74 & 0.75 & 0.78 \\
\hline reverse neighbourwise & 0.62 & 0.63 & 0.68 \\
\hline linear & 0.81 & 0.82 & 0.83 \\
\hline reverse linear & 0.35 & 0.28 & 0.44 \\
\hline non-linear & 0.61 & 0.62 & 0.68 \\
\hline reverse non-linear & 0.65 & 0.64 & 0.69 \\
\hline class 0: 30\% & 0.76 & 0.78 & 0.80 \\
\hline class 0: 20\%, class 1: 10\% & 0.77 & 0.79 & 0.82 \\
\hline class 1: 30\% & 0.76 & 0.78 & 0.70 \\
\hline class 0: 20\%, class 1: 10\% & 0.77 & 0.79 & 0.80 \\
\hline random & 0.77 & 0.79 & 0.82 \\
\hline lexicon & 0.78 & 0.73 & 0.72 \\
\hline length & 0.78 & 0.74 & 0.71 \\
\hline none & 0.84 & 0.84 & 0.86 \\
\hline
\end{tabular}

Table 3.5: Performance of Logistic Regression with different vector representations

Logistic Regression was not affected highly by the random and class dependent noise injections either. The performance with noise with the higher class imbalances did decrease the performance slightly more, as well as the length-based and lexicon-based noise. Overall, the performance of Logistic Regression in the presence of class noise was very good, and this classifier was also the least affected by the random types of noise out of the three classifiers being compared here.

The border noise injection methods overall had a larger affect on the performance, as shown in Figure 3.9, and in Table A.1. In particular, non-linear border noise had a large effect. This is an interesting result since the class boundary of the Logistic Regression is linear, one would have expected that the linear boundary noise would have displayed a higher effect.

The linear border noise had a very minor effect. However, it was actually the reversed linear border noise had the overall largest impact in reducing the performance. This suggests that examples furthest from this linear boundary are the most important to remain correctly labelled, in order to achieve a good performance by 
the Logistic Regression classifier. This classifier therefore is heavily influenced by the instances further away from the linear class boundary.

We also investigated the effects of parameter tuning on the Logistic Regression classifier, and the results are shown in Figure 3.7 and in Table 3.6. The parameters were tuned with cross validation using a grid search, to tune the strength of the regularization parameters. Regularization can be used to prevent over-fitting to a training set, and in the case of noisy training labels, this could lead to improved results. The results show that the tuning did improve the performance in most of the types of noise, as well as in the case with no noise.

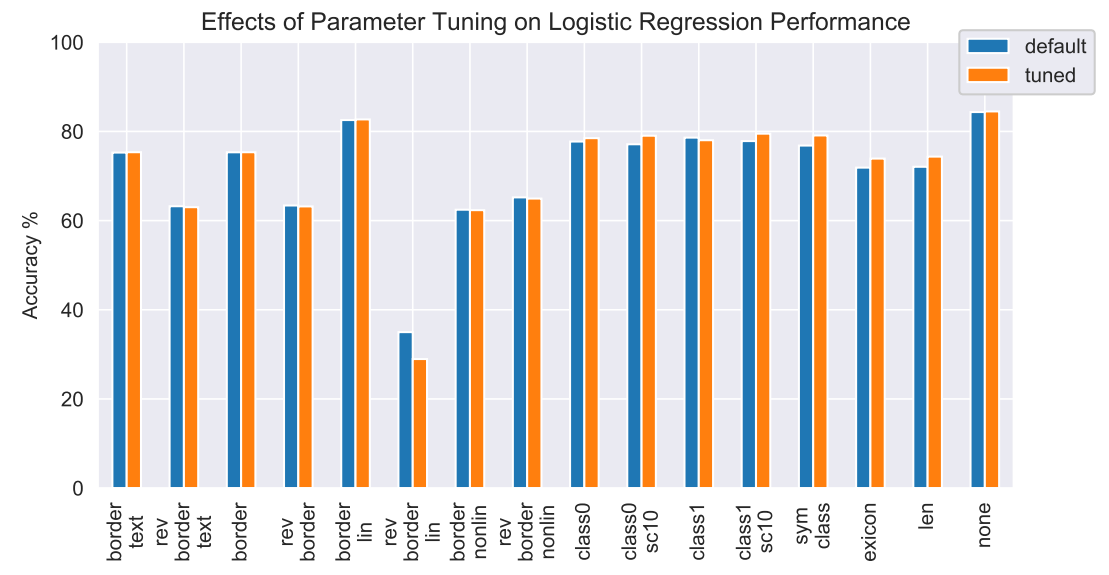

Figure 3.7: Effects of parameter tuning on Logistic Regression performance

\section{BERT}

BERT is currently a state-of-the-art method in a wide range of NLP tasks, including classification. A large part of the success of BERT-based models, is that it can make use of pre-training. There are pre-trained BERT models available, that were originally trained on extremely large datasets that required very large computational resources. A key advantage of the BERT model is the ability to fine-tune this large, pre-trained network, to tailor it to a specific task. It has been speculated that finetuning pre-trained models may also contribute to the models' noise robustness. As 


\begin{tabular}{|c|c|c|}
\hline & Default & Tuned \\
\hline \hline Text Neighbour-wise Border & 0.76 & 0.76 \\
\hline Reverse Text Neighbour-wise Border & 0.62 & 0.62 \\
\hline Neighbour-wise Border & 0.76 & 0.76 \\
\hline Reverse Neighbour-wise Border & 0.62 & 0.62 \\
\hline Linear Border & 0.81 & 0.81 \\
\hline Reverse Linear Border & 0.35 & 0.29 \\
\hline Non-Linear Border & 0.63 & 0.62 \\
\hline Reverse Non-Linear Border & 0.66 & 0.65 \\
\hline Class 0 & 0.78 & 0.79 \\
\hline Class 0 20\%, Class 1 10\% & 0.78 & 0.79 \\
\hline Class 1 & 0.78 & 0.77 \\
\hline Class 1 20\%, Class 0 10\% & 0.77 & 0.79 \\
\hline Random & 0.76 & 0.79 \\
\hline Lexicon & 0.71 & 0.72 \\
\hline Length & 0.71 & 0.73 \\
\hline None & 0.82 & 0.82 \\
\hline
\end{tabular}

Table 3.6: Performance of Logistic Regression with parameter tuning

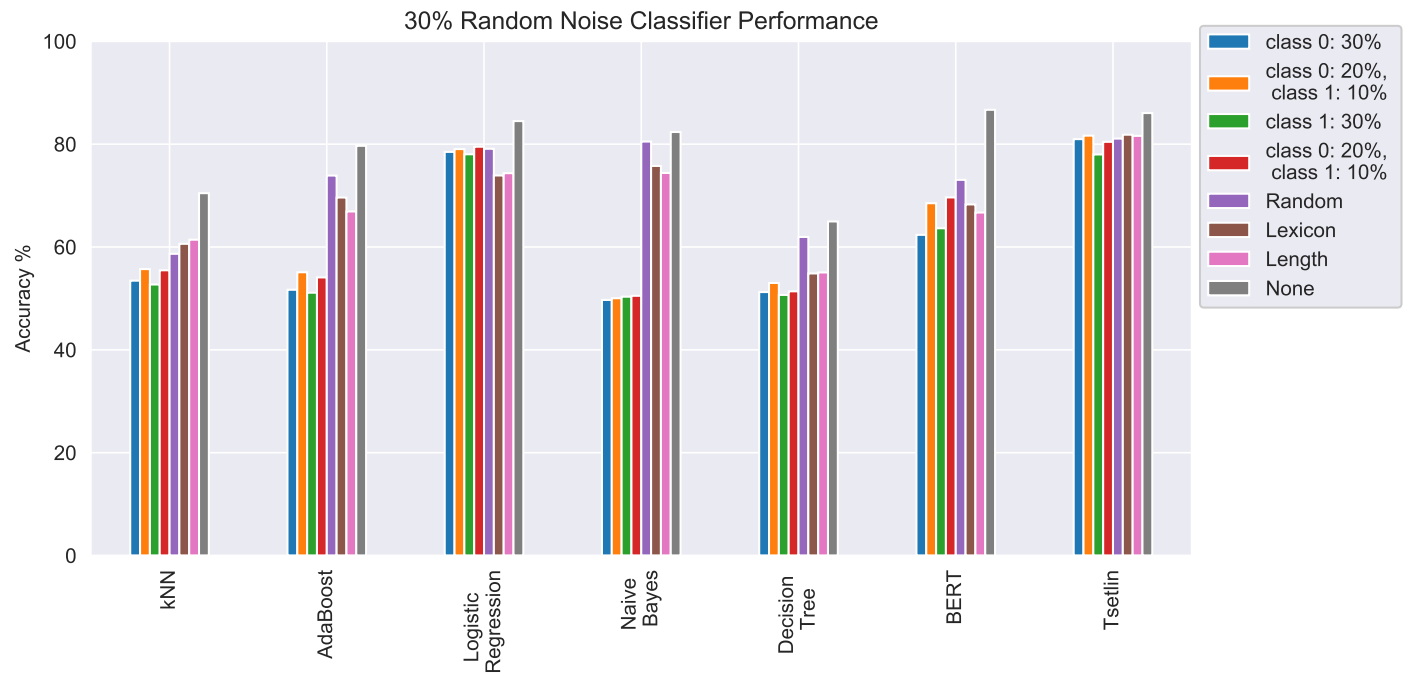

Figure 3.8: Performance of each classifier with $30 \%$ random and class dependent noise stated by Ruder and Eisenschlos [97], a hypothesis about why this model may work 


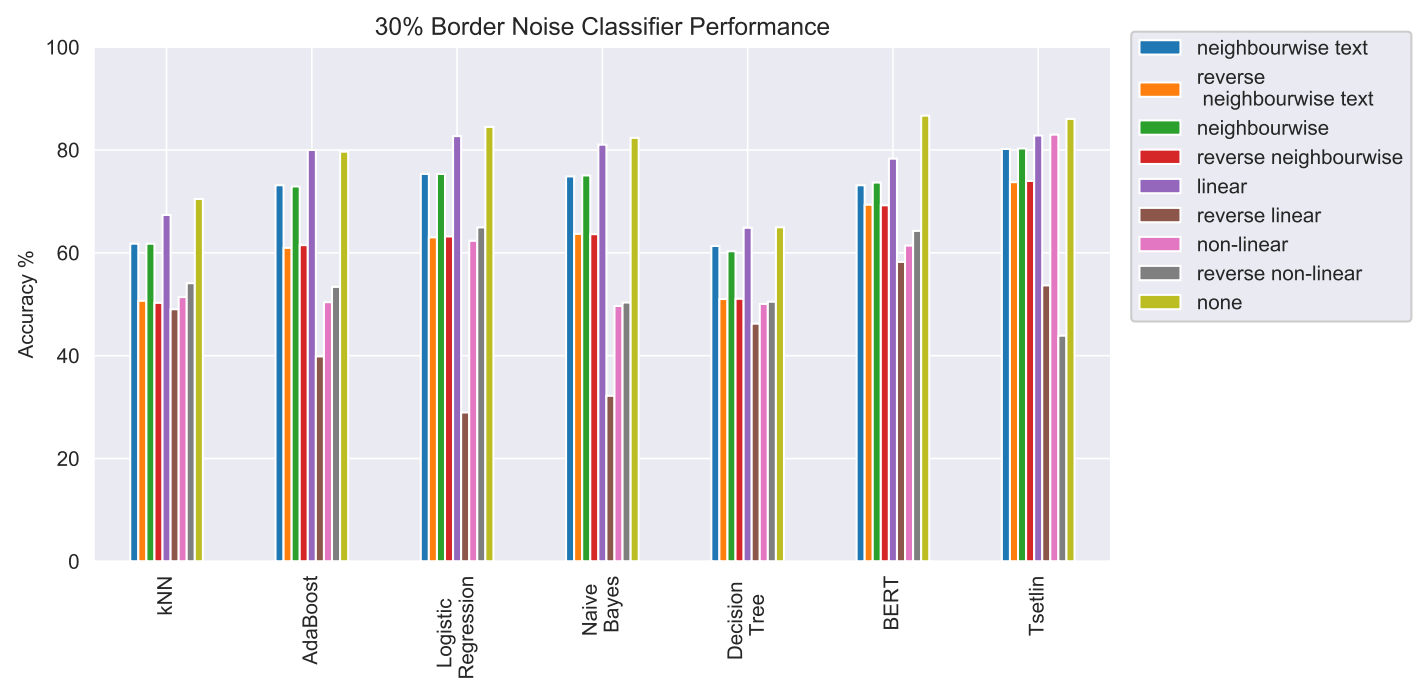

Figure 3.9: Performance of each classifier with $30 \%$ border noise

so well, is that the pre-trained information stored in the model may act as a regularizer, biasing the model towards learning correct labels, which are consistent with its existing knowledge of the language from its pre-training. Based on the results, in Figure 3.8 and Figure 3.9, BERT does perform really well, achieving the highest performance with no noise injected. With regard to it's performance with random and class-dependent noise, BERT was surprisingly highly affected. It had a larger drop in performance, more than most of the other models, notably more than the Logistic Regression classifier. This would indicate contrary to the hypothesis that the pre-training has lent some noise robustness to this model. With all the other noise types as well, BERT does experience a large drop in performance, as shown in Table A.1. Out of the injected border noise types, BERT was most affected by non-linear noise and the Reversed Linear Boundary noise.

An interesting finding was that the Reversed Linear noise affected the BERT model much less than it affected the other models, and the Non-Linear Boundary noise had a much larger affect. This is, likely, due to BERT's representation of the text documents being in a space where the class boundaries are quite different from the basic linear class boundary. Length related noise also had a big impact on the 
BERT model, which is also surprising, since BERT should have been representing the meaning of a document independently from its length. Also, the lexicon-based noise also had a quite a big effect on the performance of BERT as well.

Training the BERT model was fairly quick for a model of this size, since only fine tuning was required. For fine-tuning, typically the BERT model was trained for a duration between two to four epochs. For this experiment, the BERT model was trained for ten epochs, and the results were recorded after each epoch. These results are presented in Figure 3.10. From these figures, it is clear that BERT was able to obtain a good performance after only a single epoch of training. For the condition when there was no noise in the training labels, it can be seen that BERT reaches its highest performance after two epochs, and then the performance plateaus. For the fine-tuning of BERT in the presence of noisy training labels, it can be seen that the performance of BERT actually decreased over the epochs. This can indicate that fine-tuning with these noisy labels is actually moving BERT away from its state of containing a good representation of the documents that it had originally held encoded in its network.

\section{Tsetlin}

The Tsetlin LA classifier approach achieved quite good performance when trained on no noise, comparable to BERT's performance, as shown in Figure 3.8. This is quite an impressive result for an approach that was based on no pre-training, and based on a much more simple and interpretable representation. The performance of the Tsetlin automata with random class noise did not decrease as much as BERT, though it has not been shown to be completely robust to noise either. There are several possible reasons to explain this decrease in performance. One possibility is that the feature selection process relies on the training labels, and this process is not necessarily robust to noise. The k-best feature selection method was used as described in the original paper [9], based on the $\chi^{2}$ statistic between the feature values and the training labels. The training labels with noise may be causing a different set of features to be included in the chosen features set, than was used to achieve the high performance in the noise-free case. 


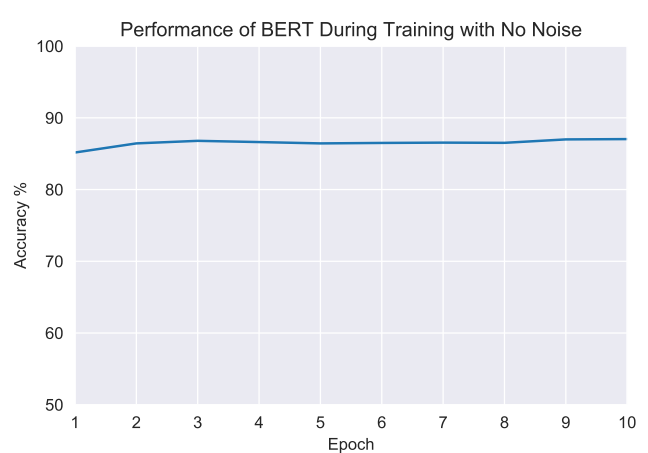

(a) Original labels

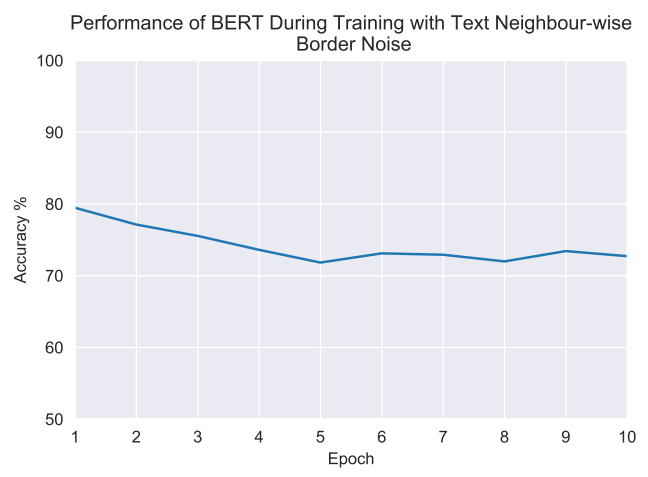

(c) Neighbourwise-border noise

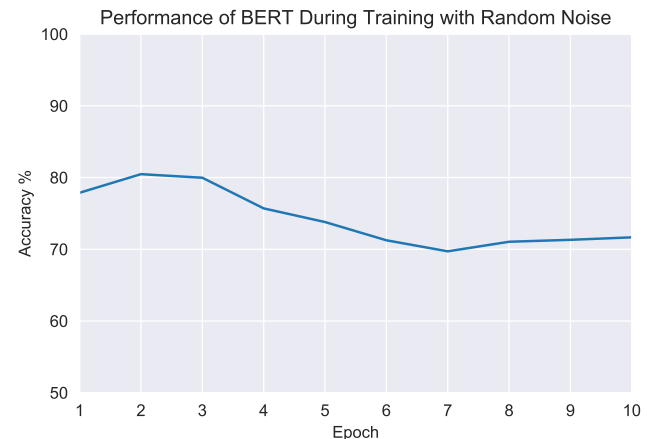

(b) Symmetric class noise

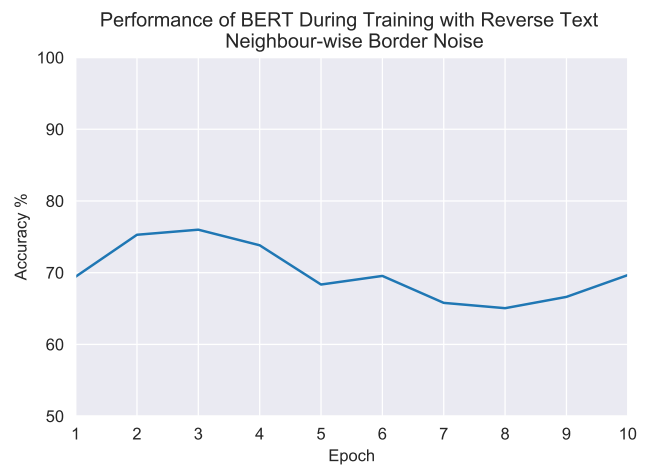

(d) Reverse neighbourwise-border noise

Figure 3.10: Training of BERT classifier with different noise types

Another possibility to explain the drop in performance could be that the LA are over-fitting to the training set with noise, due to the high dimensionality. It is well known in NLP, that terms in documents follow a Zipfian distribution, and so a large number of terms will have a very low number of occurrences. Since there were 5,000 features (terms) being used in this model, one observes that this creates a very high dimensional space. It is likely that there are many features in the training set that the LA does not have a lot of examples on, and if some of those examples had their labels corrupted by the injected noise, this could lead to an even more challenging situation for the automata to learn the optimal separation of classes in.

Following the procedure of the original paper, this model was trained for 50 epochs, and the results were tracked over time. It can be seen that the results actually 
plateaued fairly quickly, in both cases with and without the injected noise, as shown in the Figure 3.11.

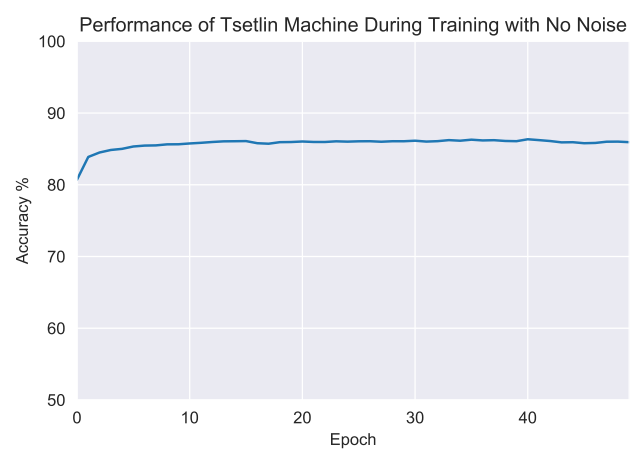

(a) Original labels

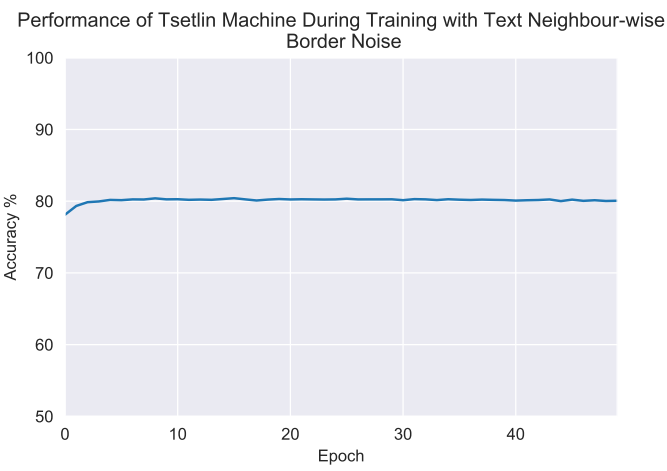

(c) Neighbourwise-border noise

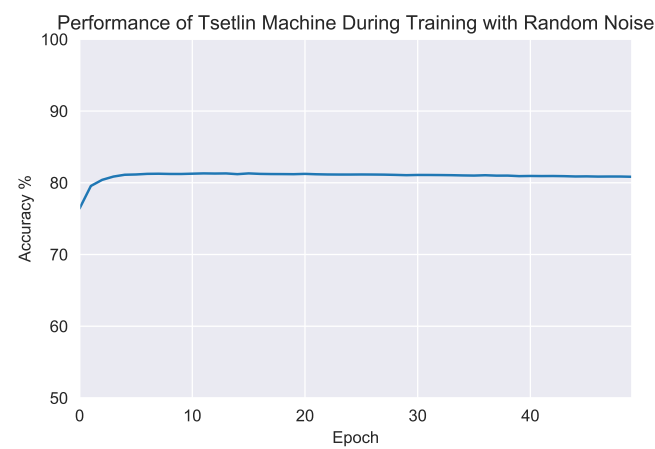

(b) Symmetric class noise

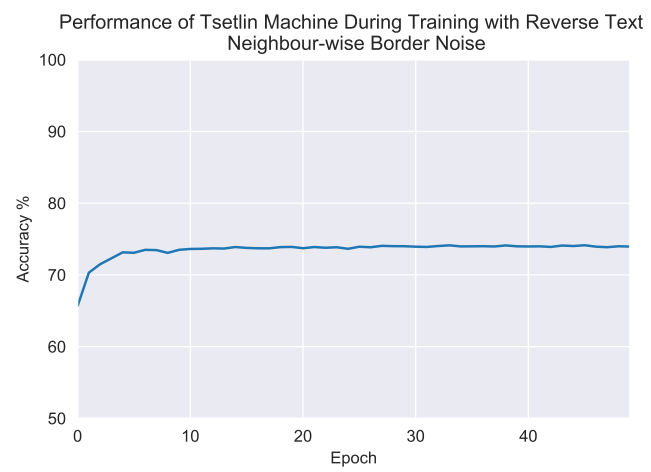

(d) Reverse neighbourwise-border noise

Figure 3.11: Training of the Tsetlin classifier with different noise types

\subsubsection{Analysis of Border Noise}

Border noise was simulated to generate more realistic types of noise. More recent research has shown that the border samples have significant information when it concerns classification, and this has lead to new nearest border classification methods. The effects of this type of noise was shown to be overall less detrimental than random noise. However, the reverse border noise was much more detrimental. The non-linear border noise was most detrimental for most of the models. The Tsetlin Machine 
performed relatively well compared to most other models in the presence of border noise.

\subsection{Conclusion}

This chapter surveyed existing classification approaches for text classification, applied to the sentiment analysis IMDb dataset. Simulations were performed to inject noise into the training labels, and then classifiers were compared with each type of noise. The objectives of these experiments were to answer the following main questions:

1. How do state-of-the-art and simple methods for text classification compare, with and without noise in the training labels?

2. How does the LA approach for text classification, the Tsetlin Machine, compare to other classification approaches with and without the presence of label noise?

3. Which types of noise would be the most detrimental to performance?

The comparison of classifiers without injected noise demonstrated that in this application of binary sentiment classification, logistic regression can perform comparably as well as the state-of-the-art model called BERT. The Tsetlin Machine also had a good performance with no noise, and was also comparable to state-of-the-art with this application. When comparing these classification methods with added random noise, we can see that the performance state-of-the-art model BERT was actually affected much more than the approach with logistic regression or the Tsetlin Machine. From these results we can conclude, that complex state-of-the-art models such as BERT should not be the go-to approach for applications with possible noise in the labels, and simple classification objectives. The advantages of these more complex models will likely be more apparent with more complex meaning to capture in the classification task, such as sarcasm, and with more challenging NLP tasks like question answering.

Various types of noise were injected into the training labels to compare these classifiers. This included random and class dependant noise, as well as novel noise injection methods that are feature specific. The border-noise did not have as big 
of an affect as the random noise, however the reversed border noise had the biggest effect on decreasing the performance. This is a good finding, because border noise would likely be the most realistic to occur in practice. Instances closest to the border should be the most similar instances, and therefore the most likely to have errors from human labeling. 


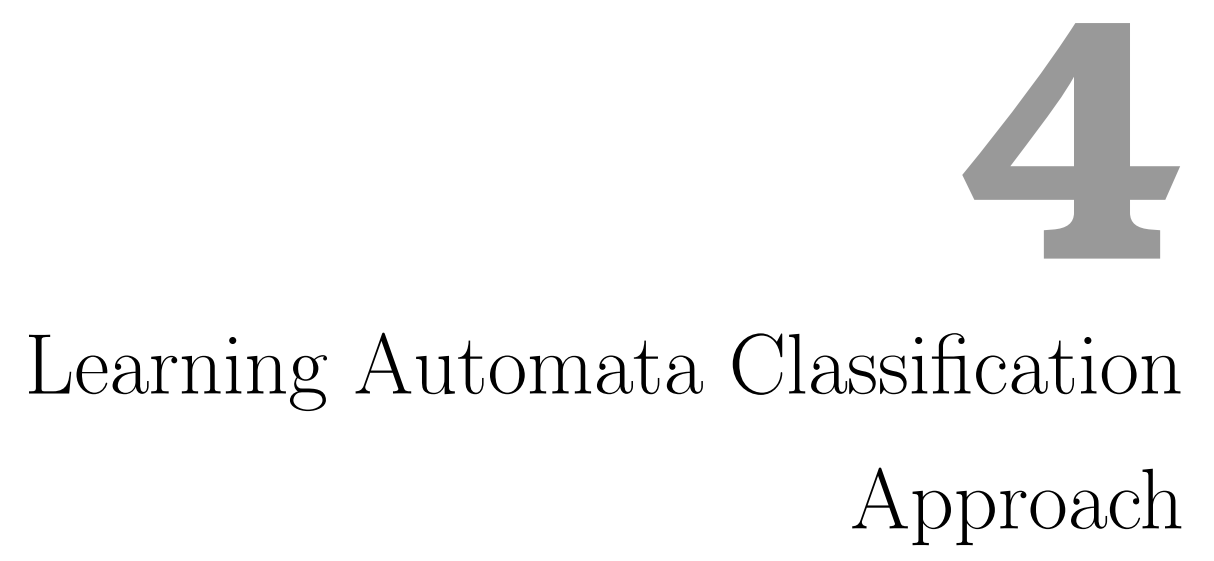

This chapter will discuss the reported LA approach for classification, including the architecture of the Tsetlin Machine's classification algorithm, the implementation, and experimental testing of its parameters. While previous sections explained the fundamental learning unit, the TA (TA), and provided explanations and experimental results on the application for text classification, this section will now focus on details of the architecture of the setup that involves that Tsetlin Machine. The Tsetlin Machine can be used for text classification, as well as for many other tasks. This chapter will provide an example application on the XOR function (as has been reported in the literature), for the purpose of investigating the effects of the Tsetlin Machine's parameters and architecture. Similar experiments will then be repeated on the IMDb text classification dataset. This will provide more insights on the Tsetlin Machine's 
learning mechanisms.

\subsection{The Tsetlin Machine Architecture}

This section will discuss more details about the implementation and algorithms behind the Tsetlin Machine's approach for classification. It will first present an overview of the method and the rationale behind this approach. Thereafter, we submit experimental results that will demonstrate the effects of the parameters.

\subsubsection{Overview}

The Tsetlin Machine, was invented by Granmo [39] in order to solve complex pattern recognition problems using TA as the base learning unit. The idea behind this approach is inspired by neural networks, which utilize artificial neurons as the base unit. Granmo proposed to use the TA as the base unit, since it is a relatively simple and versatile learning mechanism. Using the bit-wise implementation, it is possible to learn an optimal action in a stochastic environment using only a single bit of memory for each automaton. The TA serves as a good building block for complex ML tasks because of its ability to handle stochastic and unknown environments, combined with its computational simplicity and small memory footprint. The Tsetlin Machine is made up of a collection of TA, and orchestrates them to learn propositional formulae (clauses) that provide high PR accuracy. The Tsetlin Machine is a new approach to global construction of decision rules that can be learned on-line, as well as under particular noisy conditions.

In order to obtain complex problem solving strategies, one requires teams of TA. The automata independently decide upon their actions, and these decisions are directly based on the feedback from the Environment. This allows problems to be solved in a decentralized manner. However, as the number of TA grows, the level of noise increases. This effect is known as the "vanishing signal-to-noise ratio" problem. To counter this issue, a unique learning scheme, based on game theory, was designed by Granmo [39]. This scheme allows thousands of TA to successfully cooperate so as to 
learn patterns. This learning scheme, referred to as the Tsetlin Machine, was specifi-

cally designed by taking into consideration implementation decisions. This method is particularly well suited for "digital" computers, since it is directly based on bit-wise manipulations, using the simple AND, OR and NOT operators.

\subsubsection{Architecture}

The architecture of the Tsetlin Machine involves three levels:

1. Learning to identify a class: Each class is identified by evaluating a number of clauses.

2. Learning the value of a clause: The output of each clause is determined based on the included features in the clause, and the presence of these features in the input data.

3. Learning the action for a TA: Each TA represents a term in a clause, and so each TA has to learn the optimal action, namely whether to include or exclude that term.

Each of these levels of the Tsetlin Machine architecture will be explained in further detail in following sections. The high level architecture is illustrated in Figure 4.1. This figure shows the input, a vector of zeros and ones, going into numerous conjunctive clauses. The zeros and ones in the input indicate the presence or absence of a feature in the input data point. The conjunctive clauses are made up of the included terms, chosen by the TA, and evaluated based on the presence or absence of the features in the current input data point. The specific details of how the terms are chosen to be included in a clause are shown in Figure 4.2. The TA are depicted by the column of squares connected by arrows, and the chosen state is indicated with a dot. There is a TA for each feature, and the state that each TA is in determines which terms are included in the clause. Another important phenomenon to note about the architecture is the presence of a clause polarity.

From both Figure 4.1 and Figure 4.2, it can be seen that there is a positive and negative sign indication on the clauses, which indicates a positive or negative polarity, 


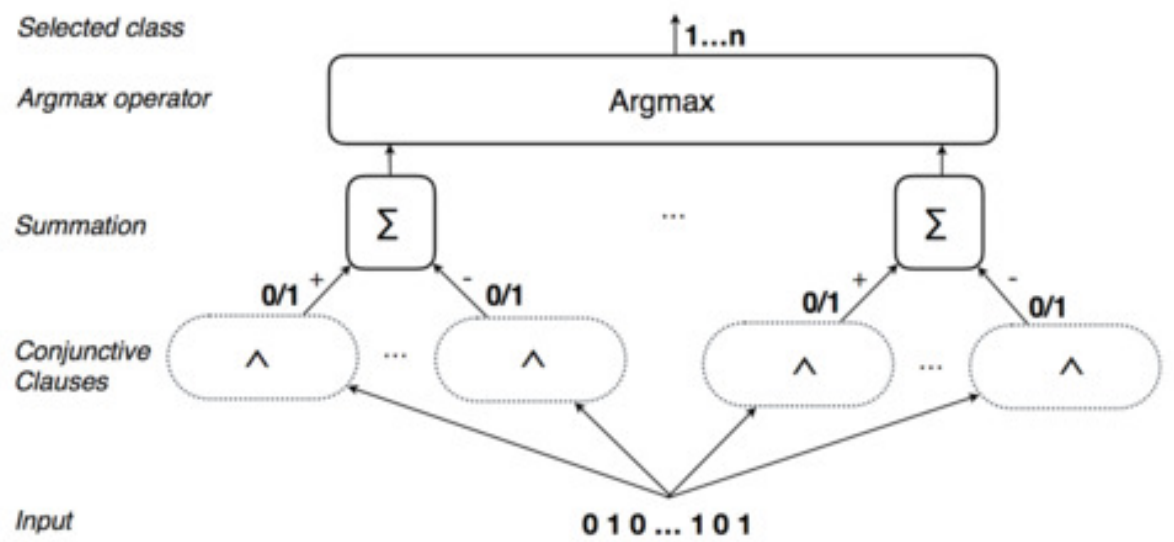

Figure 4.1: High level architecture of the Tsetlin Machine [39]

respectively. When computing the evidence for an input data point being from a particular class, the outputs of the positive clauses are summed, and the outputs of the negative polarity clauses for that class are subtracted from the score for that class. This procedure is done for all classes, and the final class chosen is determined as the one which has the highest score.

More details on how this architecture works to produce predictions will be described in Section 4.1.3, and the training algorithm is described in Section 4.1.4.

\subsubsection{Algorithm for Generating Predictions}

In this section, we describe the algorithm for producing predictions from a trained Tsetlin Machine, and the training procedure will be described in the following section, Section 4.1.4.

There are three steps involved in determining a prediction for a class for a single input data point, $x_{i}$ :

1. Evaluate each clause with the input data point.

2. Compute a sum of all the clauses to compute a score for a class.

3. Generate the final class label prediction based on the scores from each class. 


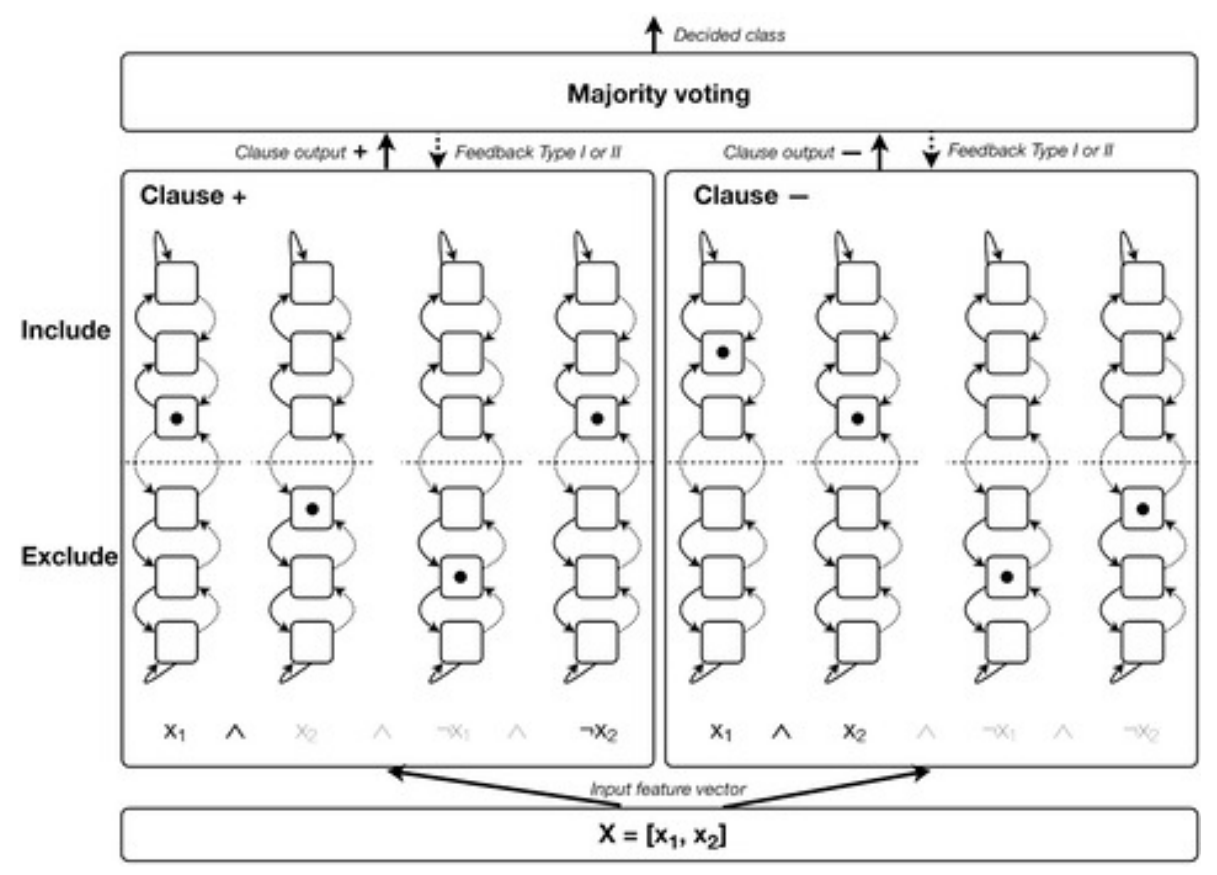

Figure 4.2: Internal architecture of the Tsetlin Machine [39]

From a high level perspective, the predicted class is determined based on comparing scores computed for each possible class, and then choosing the appropriate one based on which one has the highest score, indicated by the "Argmax" layer in Figure 4.1. In order to determine the scores for each class, one computes the outputs of many clauses. The outputs of the clauses are then used to compute the score for a class, as indicated in the following equation.

$$
f(X)=\sum_{j=1,3, \ldots}^{m-1} C_{j}(X)-\sum_{j=2,4, \ldots}^{m} C_{j}(X),
$$

where $X$ is the input data, $f(X)$ is the score for a class, $C_{j}(X)$ is clause $j$ evaluated on $X$. There will be $m$ clauses in total, where half of them have a positive polarity, and the other half have a negative polarity. The authors of [39] made an implementation decision to have all the positive clauses denoted with an odd index, and the negative polarity clauses to have an even index. These clauses are evaluated for each data point $x_{i}$ in $X$. A clause is evaluated as shown in the following equation: 


$$
C_{j}\left(x_{i}\right)=x_{i 1} \wedge x_{i 2} \wedge \ldots x_{i r} \wedge \neg x_{i r+1} \ldots \wedge \neg x_{i s}
$$

where $r$ is the number of positive features included in the clause, and $s$ is the number of negated features included in the clauses.

For further clarity, we present an example involving the evaluation of a simple clause. Considering an example input, $x_{i}=11010$, which has 5 features. The terms included in the clause, for example, could be: $x_{1} \wedge \neg x_{2} \wedge \neg x_{5}$. To evaluate this clauses given the features present in $x_{i}$, the values of features 1,2 , and 5 would be presented as input into these variables in the clause, as follows: $1 \wedge \neg 1 \wedge \neg 0$. First, by evaluating the negated features, the clause becomes, $1 \wedge 0 \wedge 1$. Since each term is joined by a logical $A N D$ operator, the output of this clause would therefore be evaluated as 0 . Therefore, if this was a positive polarity clause, since this clause evaluated to 0 , it would not contribute to the total sum for this class's score. This procedure would be repeated for each input data point, $x_{i}$, in the dataset $X$ to get a prediction of its respective class.

\subsubsection{The Training Algorithm}

In order to generate the predictions, the Tsetlin Machine must first be trained on the training set, which includes the true labels for each point. This will allow the TAs to be in the optimal states so as to choose the terms that are to be included in each clause for generating predictions. This training procedure uses the underlying mechanisms of the TAs to learn, which, as we know is capable of achieving this in noisy environments. The training algorithm follows a similar protocol as that of generating the predictions. However, after a prediction is made, the correct label from the training dataset is provided to determine whether to give a positive or negative feedback to the TAs. Once the feedback is computed for each TA, the automata are then updated, as previously described in Chapter 2, by moving its current state inwards or outwards. For more details on how the TAs operate to learn an optimal action after receiving iterative feedback, we refer the reader to the literature in Chapter 2.

This method will, clearly, involve a large number of LA. For each clause, there are 
two automata for each feature, namely, one for the feature and one for the negated feature. Therefore, if there are $n$ features, there will be $2 n$ automata for each clause. This $n$ could be quite large. For example, in the case of text classification, this number was 5,000, and in general, the number of clauses is a parameter to be chosen by the user. If there are $m$ positive polarity clauses, there will also be $m$ negative polarity clauses. Therefore for a single class, there will be $(2 n) *(2 m)$ TAs. This will be replicated for each class, and so for the case of binary classification, this will result in $2 *(2 n) *(2 m)$ TAs. In order for the reader to get an idea of the scope of the size of this method, if we use the example from text classification with 5,000 features, this therefore involves $2 * 10,000 * 2 m=40,000 * m$ TA for the learning, altogether. The value for $m$ can be selected by hyper-parameter tuning, and values chosen from past studies have set $m=80$.

It is clear that this method involves a huge number of these TA learning units, and so it will have to be implemented in an efficient way. Each of these automata receives feedback independently based on the feedback from the stochastic environment, which also poses a challenge for learning, referred to as the "vanishing signal to noise ratio". There is a novel learning mechanism invoked by the Tsetlin Machine to combat this challenge, that involves different types of feedback, and we explain this in the following section.

\section{Types of Feedback}

The feedback is determined based on whether the prediction on the training data is a true positive, a false negative, a false positive, or a true negative. The feedback scheme is designed to reduce the false negative and false positive output. This is done using two types of feedback, referred to as Type 1 and Type 2 feedbacks. The Type 1 feedback is designed to combat false negatives, and the Type 2 feedback is designed to combat false positives.

The feedback given to an individual TA depends on three factors:

- The outcome of the full clause;

- The outcome of its own term in the clause; 


\begin{tabular}{|l|c|c|c|c|c|}
\hline Truth Value of the Target Clause & \multicolumn{2}{|c|}{1} & \multicolumn{2}{|c|}{0} \\
\hline Truth Value of the Target Literal & 1 & 0 & 1 & 0 \\
\hline \hline \multirow{4}{*}{ Include Literal } & $\mathrm{P}$ (Reward) & $\frac{s-1}{s}$ & $\mathrm{NA}$ & 0 & 0 \\
& $\mathrm{P}$ (Inaction) & $\frac{1}{s}$ & $\mathrm{NA}$ & $\frac{s-1}{s}$ & $\frac{s-1}{s}$ \\
& $\mathrm{P}($ Penalty) & 0 & $\mathrm{NA}$ & $\frac{1}{s}$ & $\frac{1}{s}$ \\
\hline \multirow{4}{*}{ Exclude Literal } & $\mathrm{P}$ (Reward) & 0 & $\frac{1}{s}$ & $\frac{1}{s}$ & $\frac{1}{s}$ \\
& $\mathrm{P}$ (Inaction) & $\frac{1}{s}$ & $\frac{1}{s}$ & $\frac{1}{s}$ & $\frac{s-1}{s}$ \\
& $\mathrm{P}($ Penalty) & $\frac{s-1}{s}$ & 0 & 0 & 0 \\
\hline
\end{tabular}

Table 4.1: Type 1 feedback table

- Whether the TA has chosen to include or exclude the term.

These three factors can be collectively used to determine the feedback to the TA. The specifics of how the feedback is determined after taking into account these three factors, is shown in Table 4.1 for the Type 1 feedback, and Table 4.2 for the Type 2 feedback. Notice that the feedback scheme is probabilistic. For example, the probability of receiving a Reward from a Type 1 feedback is when the clause evaluates to 1 and the target literal evaluates to 1 , and in this case the literal is included with probabilities $\frac{s-1}{s}$. This value will be between 0 and 1 for $s>1$, and can therefore be viewed as the probability of receiving a reward. The probability of inaction in this case is $\frac{1}{s}$, and the probability of penalty is 0 . This is a unique aspect of the Tsetlin Machine that differs from the typical learning mechanism of a classic TA, since the feedback for a classic TA is only either a Reward or Penalty. Also, such a feedback protocol is determined completely by what action the TA chose, since in normal circumstances, there are no concepts of the clause or the term in the clause.

To initiate discussions, we examine the table for Type 1 feedback, Table 4.1, to gain some insights about the rationale behind this learning scheme. First of all, notice that the probability of receiving Rewards when the term or "literal" is included is larger than that of receiving Rewards when it is excluded, and the ratio between these is controlled by the parameter $s$. Therefore, the larger the value of $s$, the more terms will be included in the clauses. The effects of this parameter in a simple example will be studied empirically in the following section, Section 4.3. 


\begin{tabular}{|l|c|c|c|c|c|}
\hline Truth Value of the Target Clause & \multicolumn{2}{|c|}{1} & \multicolumn{2}{|c|}{0} \\
\hline Truth Value of the Target Literal & 1 & 0 & 1 & 0 \\
\hline \hline \multirow{4}{*}{ Include Literal } & P(Reward) & 0 & NA & 0 & 0 \\
& P(Inaction) & 1 & NA & 1 & 1 \\
& P(Penalty) & 0 & NA & 0 & 0 \\
\hline \multirow{3}{*}{ Exclude Literal } & P(Reward) & 0 & 0 & 0 & 0 \\
& P(Inaction) & 1 & 0 & 1 & 1 \\
& P(Penalty) & 0 & 1 & 0 & 0 \\
\hline
\end{tabular}

Table 4.2: Type 2 feedback table

We now examine the Type 2 feedback table, Table 4.2. The objective of this feedback type is to combat false positive predictions, which happens when the predicted value is 1 , but the target was 0 . Therefore, this type of feedback focuses on modifying clauses that evaluated to 1 , to make them evaluate to 0 in subsequent iterations, and eventually lead to the correct prediction of 0 for the class. To achieve this, we need to include more literals that are evaluated as 0 . Therefore, the TAs that have chosen to exclude a literal that evaluated to 0 will be penalized. Note that only one of these literals in the clause needs to flip from exclude to include in order for the whole clause to evaluate to 0 .

\subsection{Implementation Details}

The architecture of the Tsetlin Machine is designed with the details of its implementation taken into consideration. The implementation details will be discussed in the following sections. We first provide an overview, and this will be followed by the details of how each step is implemented.

\subsubsection{Implementation Overview}

Given that there will be thousands of TAs, as previously mentioned, the implementation of the overall machine so as to possess a small memory footprint, is critical. The implementation approach taken by the inventors, was to represent each TA on the level of bits, and to perform bit-wise manipulations for the updates. Thus the 


\begin{tabular}{|c|c|c|c|}
\hline & TA 1 & TA 2 & TA 3 \\
\hline State Bit 1 & 1 & 0 & 0 \\
\hline State Bit 2 & 0 & 0 & 1 \\
\hline State Bit 3 & 1 & 1 & 0 \\
\hline
\end{tabular}

Table 4.3: Example of a TA chunk implementation

TAs are not represented in a straightforward manner. The state of each TA is encoded as an integer. If there are 256 possible states, this could be encoded with an 8-bit integer. Half the states, from 0 to 127 , would represent one action, which is to exclude the term, while the other half of the states, from 128 to 255 , would represent the action of including the term. In order to determine the action chosen by a TA, it would only be required to check one bit in that integer, the leftmost bit which would be a 1 once the integer's value is larger than 127 . Using this fact, it is possible to check the action of many TAs in a single step. The inventors took advantage of this in their implementation. Instead of encoding the state of each TA in a single integer, they encoded states for chunks of TA together. This is illustrated in Table 4.3, which provides an example with the TA chunk size being 4, and the number of states for each TA being 8 , which can be encoded with 3 bits.

The example of the implementation of a TA chunk with chunk size of $3 \mathrm{TA}$, and each TA having 9 states, is shown in Table 4.3. In this example, TA 1 is in state 5, represented by the state bits $(1,0,1)$. This is in the larger half of the states, that would correspond to choosing action 1 . TA 2 is in state 4 , represented by the state bits $(1,0,0)$. This would correspond to this TA choosing action 0 . Finally TA 3 is in state 2 , represented by the bits $(0,1,0)$. What we can note from this, is that only the last row of this chart, State Bit 3, needs to be checked in order to determine which action each TA has chosen in the TA chunk. The inventors used this fact in their implementation, in order to encode the states for each TA in a TA chunk, row by row. Then, when it is required to check the action of the TAs, only one integer needs to be checked. This integer is used with bit-wise operations, compared with the input data, to determine the output of the clause. Further details on how such an implementation of TA is used for the main operations required, will be explained 


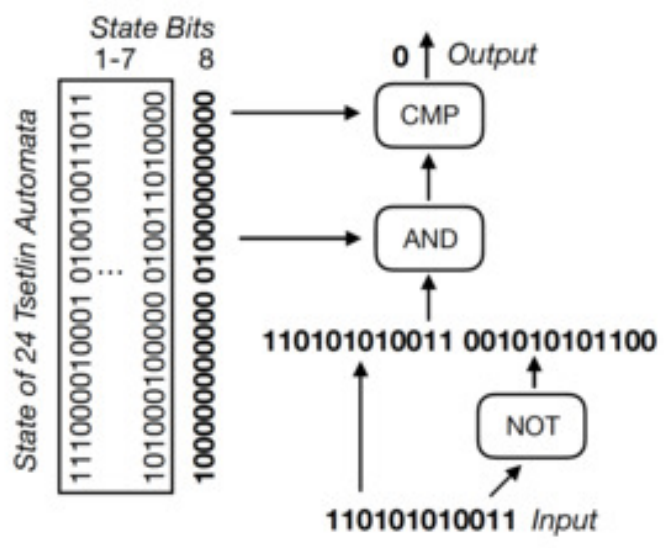

Figure 4.3: Example of a TA chunk implementation with 24 TA [39]

in the following sections.

A larger example with $24 \mathrm{TA}$ in the chunk is shown in Figure 4.3. The bit that needs to be checked in this case is bit 8. This figure also demonstrates how the bits for these TAs can be used to evaluate the clause, given the input data, which is also encoded in bits, using the bit-wise operators AND, NOT and CMP (compare). Recall that given an input, there will be two automata for each feature, one for the feature being present, and the other for the feature being negated (i.e., not present, or having the value of 0 ). This is achieved by concatenating the original input with the inverted version of the original input. Therefore, in this example, there are originally 12 features, and once they are concatenated with the inverted features, results in a total of 24 bits.

Increasing and decreasing the states of the TA can be done efficiently in parallel. This involves modifying each sequence of state bits for the TA chunk. For generating predictions after training, such an implementation method is very fast, requiring only checking the single bit that is required to indicate the action.

\subsection{XOR Simulation Experiments}

We have performed experiments on a controlled example, to further investigate the architecture of the Tsetlin Machine. The hyper-parameters previously mentioned 


\begin{tabular}{|c|c|c|}
\hline \multicolumn{2}{|c|}{ Input X } & Output Y \\
\hline 0 & 0 & 0 \\
\hline 0 & 1 & 1 \\
\hline 1 & 0 & 1 \\
\hline 1 & 1 & 0 \\
\hline
\end{tabular}

Table 4.4: XOR function

throughout the explanation of the architecture were also tested. The hyper-parameters to be investigated are: the $s$ parameter, the number of states, and the number of clauses. Each of these parameters was investigated at two different noise levels: 0.15 , and 0.3 .

\subsubsection{XOR Function}

The dataset to be used for these experiments was a simulated dataset, generated from the XOR function, which produces a 1 when the two features are different, and a 0 when the two features are the same, as illustrated in Table 4.4.

This function was used to generate synthetic data for the purpose of experiments. The synthetic data had $n$ features, where only two of these were actually used to generate the output value $y_{i}$ for each training example $x_{i}$. Consequently, there were many non-informative features. These are features that the Tsetlin Machine had to learn to not include in the clauses. The number of features used for this example were 5,000 , since this is the same as the number of features used in the text classification example. It is thus clear that there were a large number of uninformative features. This is also comparable to the case of text classification, since most terms in the vocabulary will not be involved in each clause.

Apart from the above, the XOR function outputs were injected with random noise for the training data. The noise levels used were 0.15, and 0.3. The Tsetlin Machine was trained iteratively on numerous examples, and the accuracy scores on the test data was evaluated periodically throughout the training. The results of the these scores are shown in the following sections, whence the effects of each parameter are analyzed. 


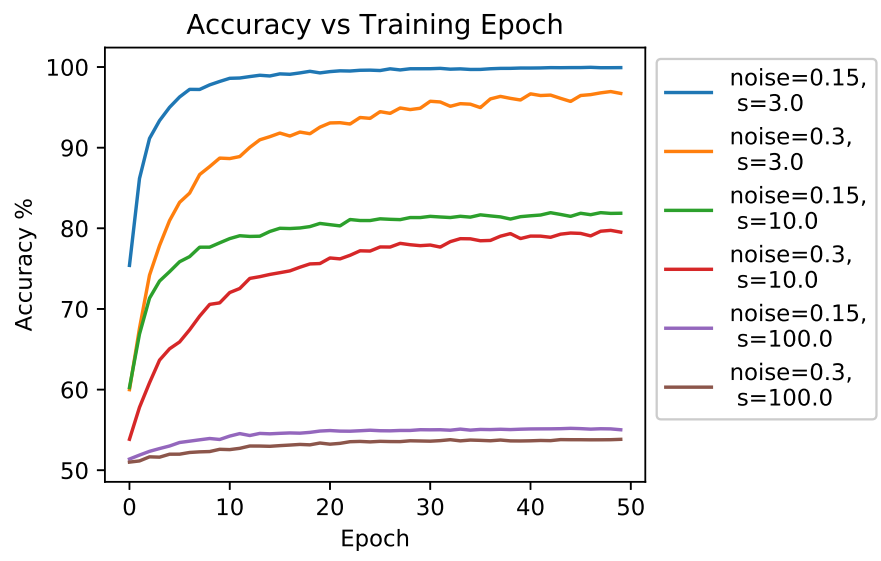

Figure 4.4: Accuracy vs epoch during training for different parameter settings for the XOR function simulated dataset

\subsubsection{Results}

The following sections will demonstrate experimental results of testing the effects of various parameters, as well as the levels of injected noise.

\section{Effect of Noise Level}

The performance of the Tsetlin Machine was assessed with two different levels of noise injected into the training labels, namely, 0.15 and 0.3 . The complete results are summarized in Table A.3 for the 0.15 noise level, and Table A.4 for the 0.3 noise level. The tables display the summarized results for each combination of the relevant parameter settings. These are the averaged results from 100 different simulated experiments. Several aspects of these results are also illustrated with plots. First, we view the effects of the level of injected noise on the test scores over time during training, in Figure 4.4, and Figure 4.5, respectively. The x-axis is denoted as "Epochs", which refers to the training on 5,000 simulated training examples. After training for 5, 000 input training points, the accuracy obtained on the test data was evaluated. Both these figures consistently show that the lower noise level accuracy plateaus at a higher accuracy than the higher noise level. 


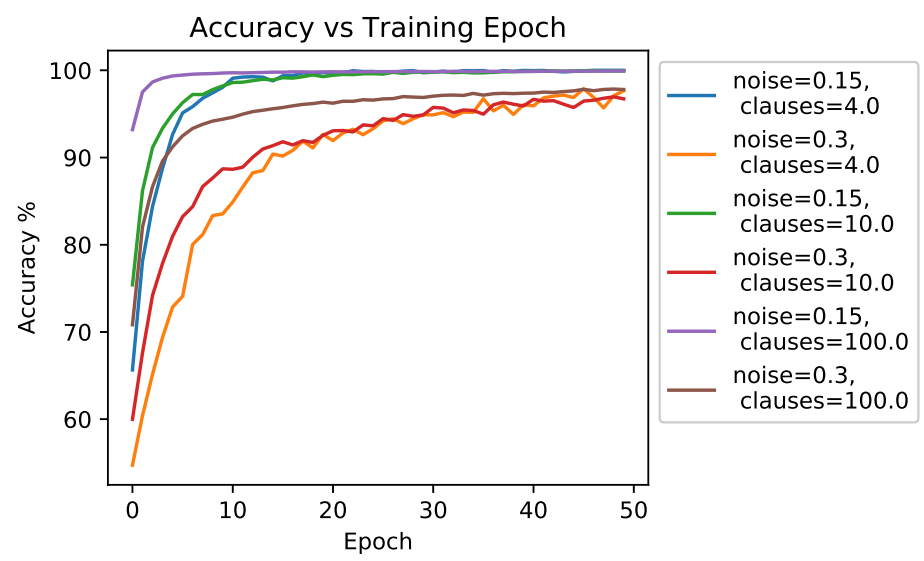

Figure 4.5: Accuracy vs epoch during training for different parameter settings for the XOR function simulated dataset

\section{Effect of Parameter $s$}

To analyze the effects of the $s$ parameter on training, Figure 4.4 displays the accuracy throughout training at three different values of $s, 3,10$, and 100 . It is clear that the lower the $s$ value, the better the performance for both noise levels. Lower values of $s$ result in more smaller clauses, as previously explained, which is consistent with what is expected for this application.

\section{Effect of Number of Clauses}

The effects of the number of clauses is illustrated in Figure 4.5. This graph shows that the number of clauses does not have a big effect on the final level of accuracy. The number of clauses tested were 4, 10 and 100. This graph, which displays the accuracy throughout training shows that the number of clauses had an effect on the amount of training examples needed before reaching the plateau of convergence to the maximum obtainable accuracy for each noise level. It can be seen that involving more clauses resulted in reaching a high accuracy much sooner in the training process. This could be due to the fact that as the number of clauses increases, the chances that some of them will capture the correct clauses to characterize the patterns, also 


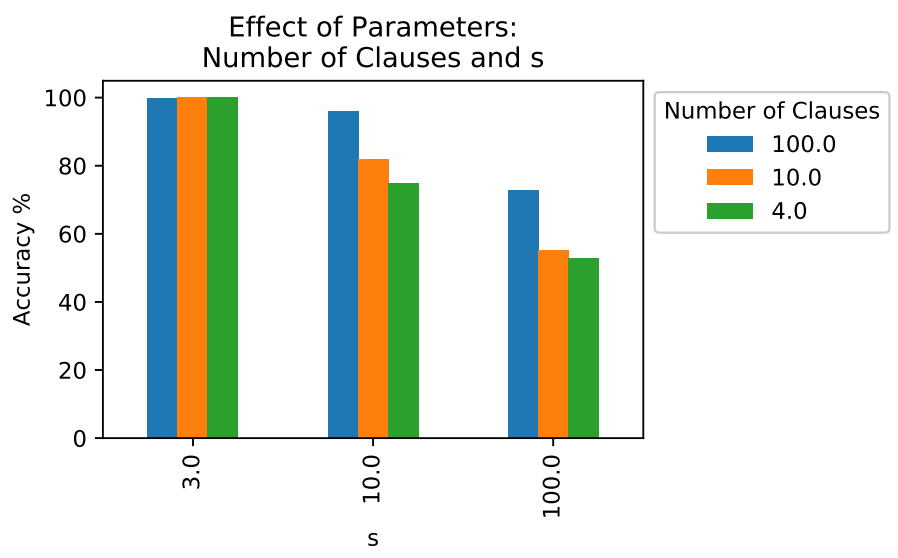

Figure 4.6: Effect of parameters s and number of clauses with the XOR dataset

increases.

The effects of the number of clauses is also summarized in Figure 4.6. This plot shows that with the low value of $s$, the number of clauses did not have an impact on the accuracy. However, with higher values of $s$, the accuracy increased with the number of clauses.

\section{Effect of Number of States}

The effects of the number of states is summarized in figure 4.7, where it is also compared with the effects of parameter $s$. Here we can once again see that a lower $s$ value resulted in the best accuracy. However, when the number of states is small, i.e., when the number of states is 16 , the value of $s$ did not have a big impact on the accuracy.

\subsection{IMDb Experiments}

This section will relate the investigations of the the architecture of the Tsetlin Machine back to the main application of text classification, which was sentiment analysis on the IMDb dataset. This section will demonstrate the results of the experiments for 


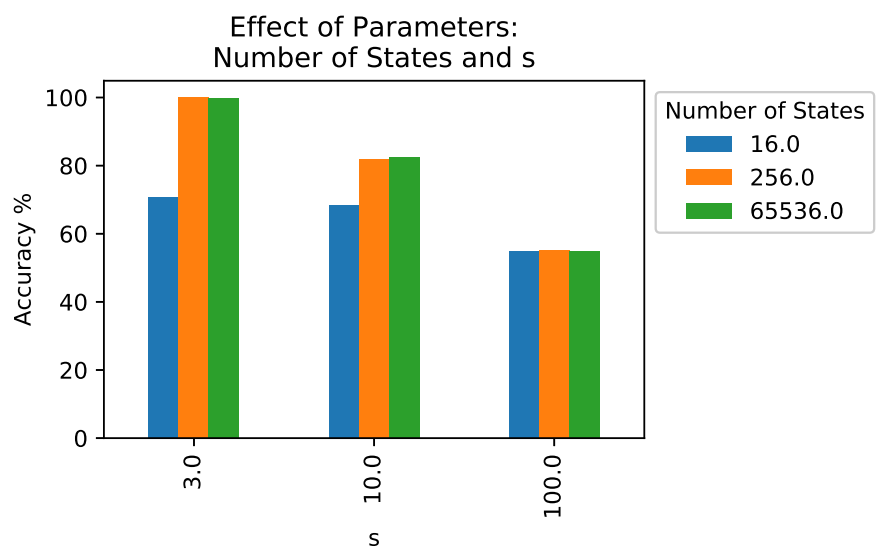

Figure 4.7: Effect of parameters s and number of states with the XOR dataset

parameter tuning, in a manner similar to what was done in the previous section.

The experiments will investigate the effects of the main parameters on the performance for text classification. The main parameters, as explained previously, are the $s$ parameter, the number of clauses and the number of states. First, we will start by testing the number of clauses. This parameter will likely be significantly different from what was obtained for the XOR data, because the nature of the features in this application is quite different. In the XOR example, we know that only two features are necessary to fully capture the XOR function. Therefore, it should be feasible to capture all the clauses possible with these two relevant features with a low number of clauses. However, in the example of text classification, the features which represent words, will be significantly less concrete, and it will likely require a much larger number of clauses to capture clauses that can characterize a sentiment, since there are many ways a person could express a positive sentiment in text. Therefore, the number of possible clauses tested for this experiment was set at a much higher level than what was previously done, and the values to be tested were 5,000, 10,000, and 15,000 .

Once we viewed the effects of this parameter, the number of clauses, we kept that value fixed and proceeded with testing the number of states. It is likely that this 


\begin{tabular}{|c|c|c|c|}
\hline Accuracy (\%) & s & Number of States & Number of Clauses \\
\hline \hline 83.64 & 3.0 & 256 & 10000 \\
\hline 84.38 & 30.0 & 256 & 5000 \\
\hline 76.75 & 30.0 & 16 & 10000 \\
\hline 86.85 & 30.0 & 256 & 10000 \\
\hline 87.54 & 30.0 & 65536 & 10000 \\
\hline 87.07 & 30.0 & 256 & 15000 \\
\hline 76.53 & 300.0 & 256 & 10000 \\
\hline
\end{tabular}

Table 4.5: Results for parameter testing on IMDb sentiment classification

parameter would be similar to what was obtained previously. The number of states, as long as there are enough, did not have a big impact. From the previous experiments, we can see the with 8 state bits and 256 states, this resulted in a performance that was just as good as when there was 16 state bits and many more states. We conjecture that this will be the case once again for this experiment.

Finally, we examined the effects of the $s$ parameter. This $s$ parameter has a big impact on the performance, as seen previously. The effects of $s$ is to introduce more randomness into the training procedure, and it also results in a higher number of smaller clauses. The higher the value of $s$, the bigger the tendency is to have longer and more complex clauses. Therefore, it is expected that for this example, the appropriate $s$ value will be much higher than for the XOR example, since the clauses used to characterize a sentiment will likely contain more than two words each, whereas the XOR function example can be characterized by using only the two features that were used in the XOR function to generate the $y$ values. A higher $s$ also reduced the amount of randomness introduced during training. For example, we can see that as $s$ approaches infinity, the probability of reward in the feedback chart in Table 4.1 would approach unity, and therefore the rewards in the training mechanism would no longer be probabilistic. It is therefore also expected that the higher values of $s$ will result in faster training.

We will investigate and confirm these effects with experiments, and the results will be shown in the following sections. A summary of the results is also shown in Table 4.5. 


\subsubsection{Effect of Number of Clauses}

We performed experiments to determine the effect of the number of clauses for the application of text classification on the IMDb dataset. The number of clauses tested were 5,000,10,000 and 15,000. This involves many more clauses than in the previous example, because it is likely that there are many possible ways to express a positive or negative sentiment using the features in the text data. Figure 4.8 shows the accuracy of the classification throughout training. The results for accuracy show that the performance for all three amounts of clauses are quite similar. Looking at the table for the precise numbers, we can see that on average, for 10,000 and 15,000 clauses the accuracy was very similar, while for 5,000 clauses the accuracy was lower by about $3 \%$. The performance, over time, with fewer clauses was lower, and was fluctuating over time, which could indicate that with too few clauses, it was less robust.

Based on these results, it is clear that the suitable choice of clauses is 10,000 . The setting with a higher number of clauses provided no benefit to the accuracy, but used twice as much memory as it was required to allocate twice as many clauses. The higher number of clauses was also lead to a slower training due to having more clauses to update at each iteration. The lower number of clauses provided a worse accuracy performance. Therefore, for this application, having 10,000 clauses seems to be an appropriate choice.

\subsubsection{Effect of Number of States}

This section will demonstrate the effects of the number of states. The number of states for each TA affects the memory required by the Tsetlin Machine. The question investigated by these tests was to see if 8 state bits was required to achieve a good performance, or if this could be achieved with fewer states. We also tested to see if adding many more states would lead to any improvement in the performance. The number of state bits being tested were 4, 8 and 16, which corresponds to 16, 256 and 65,536 states.

We can see from the experiment results shown in Figure 4.9, that with 256 states the accuracy was very similar to the performance with 65,536 states. Therefore, 


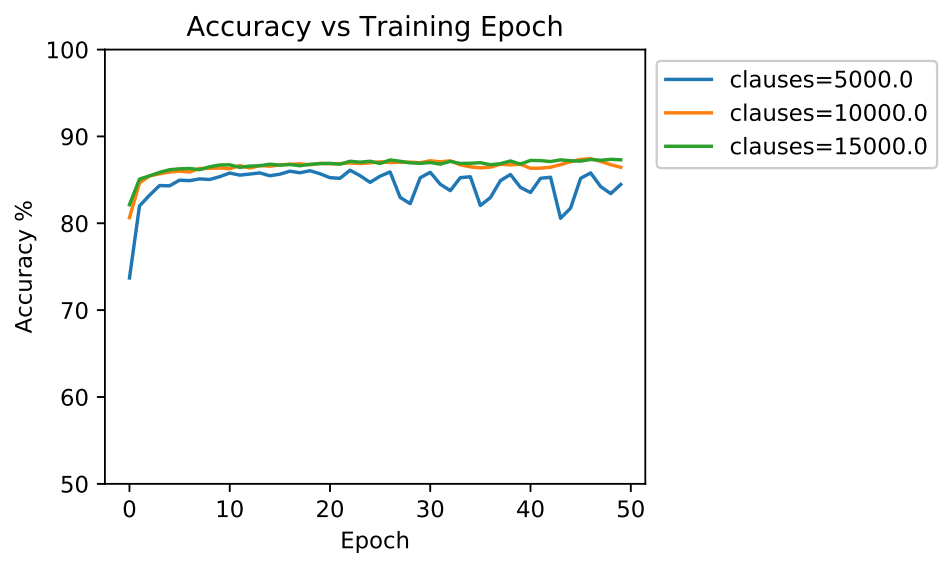

Figure 4.8: Accuracy vs epoch during training

between these two parameter options, it would be more appropriate to choose the lower number of states, since it used half as much memory, and also less computation time per training iteration, since there are lesser state bits to iterate through when making an update to the state. The lower number of states lead to a much lower accuracy. With only 16 states, there is not enough memory to allow the Tsetlin Machine to learn the clauses needed to identify the patterns in this data. Therefore the appropriate choice for the number of state bits for this application is, once again, 8.

\subsubsection{Effect of $s$ parameter}

Finally, we examined the last parameter being investigated, which is the $s$ parameter. The effects of the $s$ parameter on training accuracy over time is shown in Figure 4.10. First, examining the effects on accuracy, summarized in Table 4.5, it can be seen that the mid-range $s$ value, which was $s=30$ has the best accuracy. Figure 4.10, shows that with the parameter $s=30$, the performance plateaus and remains stable at a higher accuracy than the results for the other two $s$ parameters. This means, that with the application of text classification, clauses will be quite a bit more complex than they were for the XOR example, which had a much lower value $s$. Recall from 


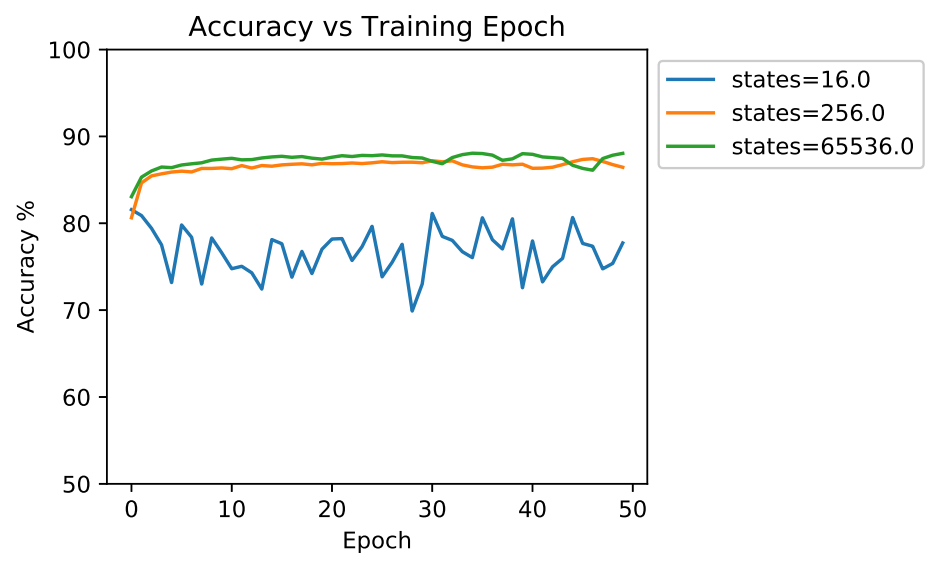

Figure 4.9: Accuracy vs epoch during training for the IMDb dataset

the explanation of the architecture of the Tsetlin Machine, a higher value of $s$ had the tendency to have longer and more complex clauses.

\subsection{Conclusion}

This chapter discussed, in detail, the architecture of the Tsetlin Machine. The Tsetlin Machine takes the learning power of the TA, and coordinates many of these base learning units to learn how to identify complex patterns in data. The scheme involves learning numerous clauses, to characterize the patterns present in the data. The clauses are represented by a large set of TA, which each is required to learn an optimal action. The experiments we did were performed on a simulated dataset, and on the text classification dataset to gain further insights on the Tsetlin Machine's architecture, and how each parameter affected the performance.

Experiments were performed on a simulated dataset for the purpose of characterising the effects of the parameters of the Tsetlin Machine. The simulated dataset was a simplified example, where the labels were generated by using an XOR function on two of the features. The dataset also included many randomly generated, uninformative features. This was a simplified example when compared to the application 


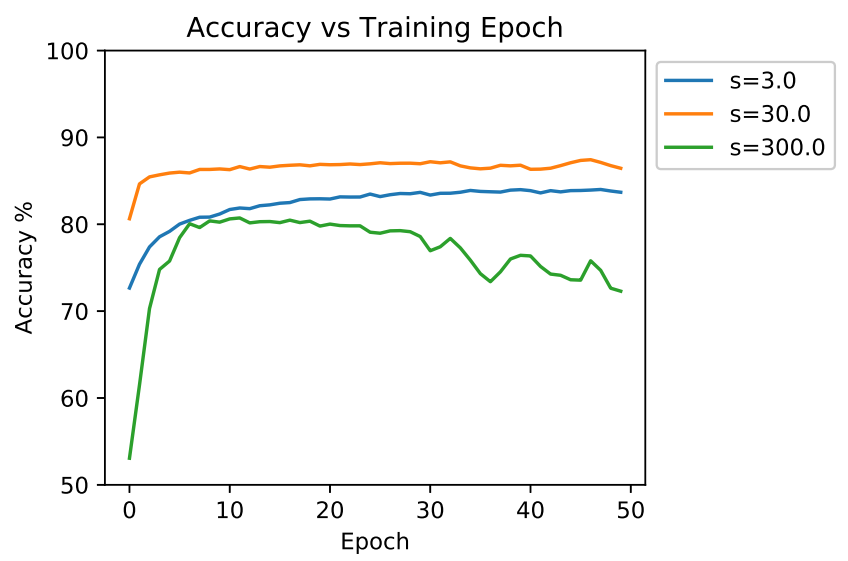

Figure 4.10: Accuracy vs epoch during training for the IMDb dataset

of text classification, which also had a large number of uninformative features, and only a few features that were required to determine how to classify a document. This dataset was generated in a controlled way, with two levels of random noise, 0.15 and 0.3 , injected into the training labels. A similar set of experiments was performed on the text classification dataset that was investigated with numerous experiments shown in Chapter 3.

The main parameters to be tested in these experiments were, the $s$ parameter which controls the randomness in the response for each TA, the number of states each TA has, and the number of clauses to be learned. The findings showed that each of these parameters affected the accuracy and the training time in different ways. Increasing the number of clauses slowed down the speed of convergence, and increased the accuracy. The $s$ parameter had a big impact on the accuracy, and an appropriate value had to be chosen for the given application. It was demonstrated that the $s$ value would need to increase in magnitude to achieve a high accuracy for more complex classification tasks. The number of states was shown to be sufficient at 256 states, so as to be able to achieve a high accuracy, and increasing the number of states past that value did not result in any improvements. The lower number of states was not able to achieve a good performance. 
These parameters also displayed interaction effects, as shown on the XOR dataset. For example, when $s$ was low, the number of clauses did not have an effect on the performance. However when $s$ was higher, it could be seen that as the number of clauses increased, the performance also increased. For the number of states, it was seen that a large number of states lead to a better performance.

Overall, we can see from these results that tuning these parameters is important for achieving a good performance from the Tsetlin Machine, and that with a good selection of parameters, learning classification patterns based on clauses can achieve a very high accuracy. These experiments provide more insights into illustrating the mechanisms of learning behind the Tsetlin Machine, as well as insights about how to select reasonable parameters for different types of data. 


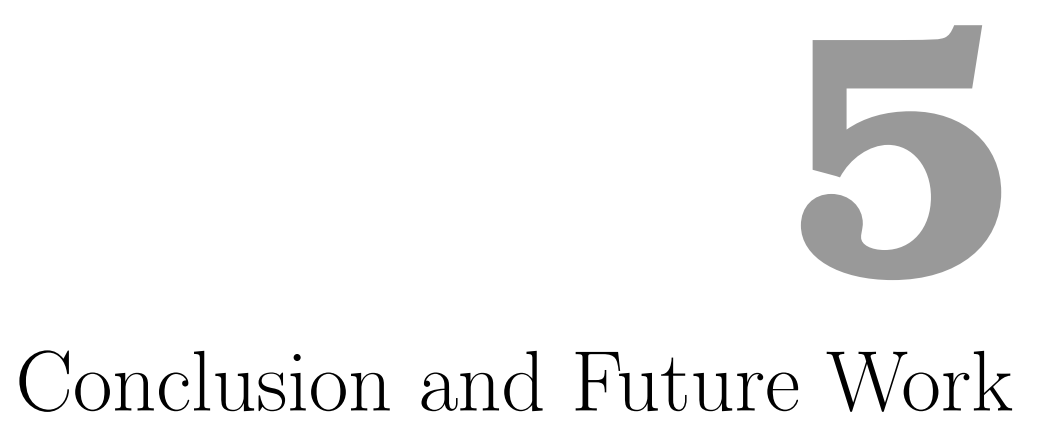

In this concluding chapter, we provide a brief summary of the thesis. We will summarize what has been done, and present conclusions on the results that we have obtained. Finally, we will provide some ideas for future work relevant to this line of research.

\subsection{Summary and Conclusions}

The main questions investigated by this thesis are:

1. How do novel and traditional classification methods compare for the application of text classification with noisy class labels?

2. What are the impacts of different types of label noise on the relative performance of these classifiers? 
3. How can learning automata be used for text classification?

A summary of the conclusions for each of these questions will be discussed in the following sections.

\subsubsection{How do Classification Methods Compare?}

The comparison of classifiers without injected noise demonstrated that in this application of binary classification, Logistic Regression and the BERT model can perform, comparably well. The novel LA approach, the Tsetlin Machine, also had a compara-

ble performance. When comparing these classification methods with added random noise, we observe that the performance of the model BERT was actually affected much more than logistic regression or the Tsetlin Machine. From these results we can conclude, that complex state-of-the-art models such as BERT should not be the default approach for applications with possible noise in the labels, and for simple classification objectives such as binary sentiment analysis. The advantages of these more complex models will likely be more apparent when it is necessary to capture more complex "meanings", such as sarcasm, and with more challenging NLP tasks like question answering.

\subsubsection{What Impacts do Different Types of Label Noise have?}

Various types of noise were injected into the training labels to compare these classifiers. This included random and class dependant noise, as well as novel noise injection methods that were feature specific. The border noise types did not have as large an effect as the random noise. However, the reversed border noise had the most-significant effect on decreasing the performance. This is a noteworthy finding, because border noise would likely be the most realistic one to occur, in practice, and it has been shown that it is not greatly detrimental. The noise injection experiments also indicated differences in models that would not have been apparent from the original scores alone, since with no noise, several models had very similar performance. With the injected noise, differences between these models were observed. 


\subsubsection{How can Learning Automata be used for Classification with Noisy Labels?}

This thesis discussed, in detail, the architecture of the Tsetlin Machine, and how it can be applied for the domain of text classification. The findings from the experiments in Chapter 4 showed that with proper the tuning of its parameters, the Tsetlin Machine can achieve a good performance on two different classification tasks. The effects of the parameters were summarized with respect to both the accuracy and the memory and training times. The main findings were that setting the number of clauses as a high value will likely be detrimental in memory and training time, but not in accuracy. The $s$ parameter should be tuned to find the most suitable value for the given application, as it is related to the complexity of the clauses that will be learned to characterize the classes. The number of states also can be set at a high value without being detrimental to the accuracy. However, choosing 256 states for this setting was suitable for both applications, and a much higher value did not lead to any noticeable improvement in accuracy.

In comparison with other classification methods, shown in Chapter 3, the Tsetlin Machine demonstrated comparable performance with the state-of-the-art methods. With various types of noise tested in the experiments, the Tsetlin Machine performed comparably well, and did not demonstrate a large drop in accuracy even at the 0.3 level of noise.

Overall, we can see from these results that tuning these parameters is important for achieving a good performance from the Tsetlin Machine, and that with a good selection of parameters, learning classification patterns based on clauses can achieve a very high accuracy that is comparable with the state-of-the-art and traditional methods, even in the presence of label noise.

\subsection{Future Work}

The main aspects investigated in this thesis could each be continued with further experimentation. Future work on these topics include experimenting with the Tsetlin 
Machine to improve its performance, experimenting with different ways to use LA for text classification, and finally experimenting to determine additional ways to improve classification performance in the presence of label noise. Each of these will be discussed in more detail in the following sections.

\subsubsection{Modifying the Base Automata in the Tsetlin Machine}

The Tsetlin Machine has a base learning unit LA, which is the Tsetlin automata (TA). The TA is a fundamental LA, and was the first LA that was invented. Since its invention, there have been many other automata developed, that have proven improvements over the TA in terms of performance and the time required for convergence. An overview of the different types of LA will be provided in the following section, as well as more details on the variable structure automata, and how these automata could be implemented in a similar architecture as the Tsetlin Machine.

\section{Overview of types of LA}

The two main types of LA are the fixed structure and variable structure automata. As described in Chapter 2, LA are characterized by their transition function, $F$, and their output function, $G$. The probability of a LA transitioning from one state to another is determined by the $F$ function. Some LA will have transition probabilities that are fixed, and these are referred to as Fixed Structure Stochastic Automata (FSSA). The other type of LA, referred to as Variable Structure Stochastic Automata (VSSA), will have transition probabilities that change over time. The Tsetlin automata is a FSSA, and there are several variants of it that are also FSSA, called such as Krinsky and the Krylov Automaton. VSSA have transition probabilities that are not constant. They can be updated at every time instant after receiving feedback from the Environment. There are several sub-types of VSSA, each of which have different performance and convergence characteristics, as briefly stated below. 


\section{VSSA implementation considerations}

The action probabilities of a VSSA are updated as the automata interacts with the environment. An example of a VSSA is the Linear Reward-Inaction $\left(L_{R I}\right)$, explained in detail in Chapter 2. This LA is absolutely expedient and $\varepsilon$-optimal. Since this LA has such good a performance characteristics, it would be interesting to test this as the base automata within the architecture of the Tselin Machine.

As previously described, the Tsetlin Machine was designed with implementation in mind. For example, recall the bit-wise manipulations involved in checking the state, as described in Chapter 4. This would not be possible with the $L_{R I}$. The $L_{R I}$ does not have the same state structure as the FSSA. The $L_{R I}$ is represented by probability values that dictate how to choose an action. These probabilities would be represented as floating point variables and updated by multiplying these probabilities, which would be a fundamentally different type of calculation than the Tsetlin Machine was designed for. Implementing this VSSA would involve drastic changes to the implementation, and therefore does not take advantage of the same bit-wise manipulations that the Tsetlin Machine was designed for.

In order to solve this issue, one could also consider the Discretized $L_{R I}\left(D L_{R I}\right)$. The discretized version of the $L_{R I}$ has performance benefits, because it reduces the slow asymptotic convergence approach to the optimal action. The $D L_{R I}$ also has a structure that involves states, that can make use of the same bit-wise operations for checking the state, as is fundamental to the design of the Tsetlin Machine. The movement from state to state would be essentially the same procedure as is described in the Tsetlin Machine.

However, challenges still remain in adapting the $D L_{R I}$ for use as the base LA in the Tsetlin Machine architecture. The $D L_{R I}$ involves inherent randomness introduced in the selection of the action, based on the probability of the current state that it is in. The Tsetlin Machine architecture's feedback charts also introduce additional randomness in the way the automata respond to the feedback. This additional level of randomness may introduce too much noise, and lead to a convergence to non-optimal actions. The feedback chart would need to be re-assessed for the purpose of using the $D L_{R I}$ as the base LA. For example, preliminary steps that could be taken, would be 
to remove the parameter $s$, so as to eliminate the randomness in the responses to the feedback.

\subsubsection{Learning Automata Approaches for Text Classification}

One challenge with the existing Tsetlin Machine approach for text classification, is that it relies on a feature selection pre-processing step, before the Tsetlin Machine comes into play for the classification task. This feature selection is based directly on the training labels, and therefore, would be susceptible to choosing non-optimal features, when these training labels have noise. A possibility by which one could solve this issue, would be to use LA for the feature selection process as well. Very few studies have been done on this so far. However, there is one study, by Seyyedi and Minaei-Bidgoli [106] that studied the use of estimator automata for the feature subset selection, with the application to email spam detection. This paper demonstrates that such an approach is a promising idea, and that it could be used for further investigations. Other, traditional, NLP methods could also be used to improve the feature selection process, such as perhaps using an input topic vector obtained from Latent Dirichlet Allocation, instead of a vector corresponding directly to words.

\subsubsection{Characterization of Types of Noise in Text Datasets}

Finally, to bridge the gap between the experimental results and real world applications, there needs to be additional research investigating which types of noise are present in real applications. This has been addressed by the Confident Learning method previously described, though this method has challenges detecting more realistic noise types. Further research would be required to detect novel noise types, such as whether border noise is present. 


\section{Bibliography}

[1] Joaquín Abellán and Andrés R Masegosa. Bagging schemes on the presence of class noise in classification. Expert Systems with Applications, 39(8):6827-6837, 2012 .

[2] Charu C Aggarwal and ChengXiang Zhai. A survey of text classification algorithms. In Mining text data, pages 163-222. Springer, 2012.

[3] Dana Angluin and Philip Laird. Learning from noisy examples. Machine Learning, 2(4):343-370, 1988.

[4] Sujeevan Aseervatham, Anestis Antoniadis, Éric Gaussier, Michel Burlet, and Yves Denneulin. A sparse version of the ridge logistic regression for large-scale text categorization. Pattern Recognition Letters, 32(2):101-106, 2011.

[5] Javed A Aslam and Scott E Decatur. On the sample complexity of noise-tolerant learning. Information Processing Letters, 57(4):189-195, 1996.

[6] Richard J Beckman and R Dennis Cook. Outliers. Technometrics, 25(2):119$149,1983$.

[7] Beata Beigman Klebanov and Eyal Beigman. From annotator agreement to noise models. Computational Linguistics, 35(4):495-503, 2009.

[8] Kristin P Bennett and Colin Campbell. Support vector machines: hype or hallelujah? Acm Sigkdd Explorations Newsletter, 2(2):1-13, 2000. 
[9] Geir Thore Berge, Ole-Christoffer Granmo, Tor Oddbjørn Tveit, Morten Goodwin, Lei Jiao, and Bernt Viggo Matheussen. Using the tsetlin machine to learn human-interpretable rules for high-accuracy text categorization with medical applications. arXiv preprint arXiv:1809.04547, 2018.

[10] Geir Thore Berge, Ole-Christoffer Granmo, Tor Oddbjørn Tveit, Morten Goodwin, Lei Jiao, and Bernt Viggo Matheussen. Using the tsetlin machine to learn human-interpretable rules for high-accuracy text categorization with medical applications. CoRR, abs/1809.04547, 2018.

[11] Yingtao Bi and Daniel R Jeske. The efficiency of logistic regression compared to normal discriminant analysis under class-conditional classification noise. Journal of Multivariate Analysis, 101(7):1622-1637, 2010.

[12] Piotr Bojanowski, Edouard Grave, Armand Joulin, and Tomas Mikolov. Enriching word vectors with subword information. Transactions of the Association for Computational Linguistics, 5:135-146, 2017.

[13] Pavel Brazdil and Peter Clark. Learning from imperfect data. In Machine Learning, Meta-Reasoning and Logics, pages 207-232. Springer, 1990.

[14] Carla E Brodley and Mark A Friedl. Identifying mislabeled training data. Journal of artificial intelligence research, 11:131-167, 1999.

[15] Carla E Brodley, Mark A Friedl, et al. Identifying and eliminating mislabeled training instances. In Proceedings of the National Conference on Artificial Intelligence, pages 799-805, 1996.

[16] Daniel Cer, Yinfei Yang, Sheng-yi Kong, Nan Hua, Nicole Limtiaco, Rhomni St John, Noah Constant, Mario Guajardo-Cespedes, Steve Yuan, Chris Tar, et al. Universal sentence encoder. arXiv preprint arXiv:1803.11175, 2018.

[17] Varun Chandola, Arindam Banerjee, and Vipin Kumar. Anomaly detection: A survey. ACM computing surveys (CSUR), 41(3):15, 2009.

[18] Wikimedia Commons. Svm margin, 2018. File:SVM margin.png. 
[19] Peter Congdon. Bayesian statistical modelling, volume 704. John Wiley \& Sons, 2007.

[20] Gordon V Cormack and Aleksander Kolcz. Spam filter evaluation with imprecise ground truth. In Proceedings of the 32nd international ACM SIGIR conference on Research and development in information retrieval, pages 604-611. ACM, 2009.

[21] Corinna Cortes and Vladimir Vapnik. Support-vector networks. Machine learning, 20(3):273-297, 1995.

[22] Sarah Jane Delany, Nicola Segata, and Brian Mac Namee. Profiling instances in noise reduction. Knowledge-Based Systems, 31:28-40, 2012.

[23] Xuelian Deng, Yuqing Li, Jian Weng, and Jilian Zhang. Feature selection for text classification: A review. Multimedia Tools and Applications, 78(3):3797$3816,2019$.

[24] Jacob Devlin, Ming-Wei Chang, Kenton Lee, and Kristina Toutanova. BERT: pre-training of deep bidirectional transformers for language understanding. CoRR, abs/1810.04805, 2018.

[25] Jacob Devlin, Ming-Wei Chang, Kenton Lee, and Kristina Toutanova. Bert: Pre-training of deep bidirectional transformers for language understanding. arXiv preprint arXiv:1810.04805, 2018.

[26] Thomas G Dietterich. An experimental comparison of three methods for constructing ensembles of decision trees: Bagging, boosting, and randomization. Machine learning, 40(2):139-157, 2000.

[27] Andres Folleco, Taghi M Khoshgoftaar, Jason Van Hulse, and Lofton Bullard. Identifying learners robust to low quality data. In 2008 IEEE International Conference on Information Reuse and Integration, pages 190-195. IEEE, 2008. 
[28] Benoît Frénay, Gauthier Doquire, and Michel Verleysen. Feature selection with imprecise labels: Estimating mutual information in the presence of label noise. Comput. Stat. Data An., submitted for publication, 2014.

[29] Benoît Frénay, Ata Kabán, et al. A comprehensive introduction to label noise.

[30] Benoit Frénay and Michel Verleysen. Classification in the presence of label noise: a survey. IEEE transactions on neural networks and learning systems, 25(5):845-869, 2013.

[31] Anil Gaba. Inferences with an unknown noise level in a bernoulli process. Management science, 39(10):1227-1237, 1993.

[32] Anil Gaba and Robert L Winkler. Implications of errors in survey data: a bayesian model. Management Science, 38(7):913-925, 1992.

[33] Dragan Gamberger and Nada Lavrač. Conditions for occam's razor applicability and noise elimination. In European Conference on Machine Learning, pages 108-123. Springer, 1997.

[34] Dragan Gamberger, Nada Lavrac, and Ciril Groselj. Experiments with noise filtering in a medical domain. In ICML, pages 143-151, 1999.

[35] Luas Paulo Garcia, Jens Lehmann, Andre de Carvalho, and Ana Lorena. New label noise injection methods for the evaluation of noise filters. Knowledge-Based Systems, 163, 092018.

[36] Luís PF Garcia, Jens Lehmann, Andre CPLF de Carvalho, and Ana C Lorena. New label noise injection methods for the evaluation of noise filters. KnowledgeBased Systems, 163:693-704, 2019.

[37] Geoffrey Gates. The reduced nearest neighbor rule (corresp.). IEEE transactions on information theory, 18(3):431-433, 1972.

[38] Richard Gerlach and James Stamey. Bayesian model selection for logistic regression with misclassified outcomes. Statistical Modelling, 7(3):255-273, 2007. 
[39] Ole-Christoffer Granmo. The tsetlin machine-a game theoretic bandit driven approach to optimal pattern recognition with propositional logic. arXiv preprint arXiv:1804.01508, 2018.

[40] Tom Heskes. The use of being stubborn and introspective. In Prerational Intelligence: Adaptive Behavior and Intelligent Systems Without Symbols and Logic, Volume 1, Volume 2 Prerational Intelligence: Interdisciplinary Perspectives on the Behavior of Natural and Artificial Systems, Volume 3, pages 1184-1200. Springer, 2000.

[41] Ray J Hickey. Noise modelling and evaluating learning from examples. Artificial Intelligence, 82(1-2):157-179, 1996.

[42] Victoria Hodge and Jim Austin. A survey of outlier detection methodologies. Artificial intelligence review, 22(2):85-126, 2004.

[43] Nicholas P Hughes, Stephen J Roberts, and Lionel Tarassenko. Semi-supervised learning of probabilistic models for ecg segmentation. In The 26th Annual International Conference of the IEEE Engineering in Medicine and Biology Society, volume 1, pages 434-437. IEEE, 2004.

[44] Stanisław Jastrzebski, Damian Leśniak, and Wojciech Marian Czarnecki. How to evaluate word embeddings? on importance of data efficiency and simple supervised tasks. arXiv preprint arXiv:1702.02170, 2017.

[45] Piyasak Jeatrakul, Kok Wai Wong, and Chun Che Fung. Data cleaning for classification using misclassification analysis. Journal of Advanced Computational Intelligence and Intelligent Informatics, 14(3):297-302, 2010.

[46] Shuiwang Ji and Jieping Ye. Generalized linear discriminant analysis: a unified framework and efficient model selection. IEEE Transactions on Neural Networks, 19(10):1768-1782, 2008.

[47] Wenxin Jiang. Some theoretical aspects of boosting in the presence of noisy data. In Proceedings of the Eighteenth International Conference on Machine Learning. Citeseer, 2001. 
[48] Rie Johnson and Tong Zhang. Effective use of word order for text categorization with convolutional neural networks. arXiv preprint arXiv:1412.1058, 2014.

[49] Lawrence Joseph, Theresa W Gyorkos, and Louis Coupal. Bayesian estimation of disease prevalence and the parameters of diagnostic tests in the absence of a gold standard. American journal of epidemiology, 141(3):263-272, 1995.

[50] Lawrence Joseph, Theresa W Gyorkos, and Louis Coupal. Bayesian estimation of disease prevalence and the parameters of diagnostic tests in the absence of a gold standard. American journal of epidemiology, 141(3):263-272, 1995.

[51] Suzan Kantarci-Savas and Efendi N. Nasibov. Fuzzy id3 algorithm on linguistic dataset by using wabl defuzzification method. 2017 IEEE International Conference on Fuzzy Systems (FUZZ-IEEE), pages 1-5, 2017.

[52] Taghi M Khoshgoftaar and Pierre Rebours. Generating multiple noise elimination filters with the ensemble-partitioning filter. In Proceedings of the 2004 IEEE International Conference on Information Reuse and Integration, 2004. IRI 2004., pages 369-375. IEEE, 2004.

[53] Taghi M Khoshgoftaar, Jason Van Hulse, and Amri Napolitano. Supervised neural network modeling: an empirical investigation into learning from imbalanced data with labeling errors. IEEE Transactions on Neural Networks, 21(5):813-830, 2010.

[54] Kamran Kowsari, Kiana Jafari Meimandi, Mojtaba Heidarysafa, Sanjana Mendu, Laura Barnes, and Donald Brown. Text classification algorithms: A survey. Information, 10(4):150, 2019.

[55] Abba Krieger, Chuan Long, and Abraham Wyner. Boosting noisy data. In ICML, pages 274-281, 2001.

[56] Taku Kudo and John Richardson. Sentencepiece: A simple and language independent subword tokenizer and detokenizer for neural text processing. CoRR, abs/1808.06226, 2018. 
[57] Peter A Lachenbruch. Discriminant analysis when the initial samples are misclassified. Technometrics, 8(4):657-662, 1966.

[58] Peter A Lachenbruch. Note on initial misclassification effects on the quadratic discriminant function. Technometrics, 21(1):129-132, 1979.

[59] Martin Ladouceur, Elham Rahme, Christian A Pineau, and Lawrence Joseph. Robustness of prevalence estimates derived from misclassified data from administrative databases. Biometrics, 63(1):272-279, 2007.

[60] Chuck P Lam and David G Stork. Evaluating classifiers by means of test data with noisy labels. In IJCAI, volume 3, pages 513-518, 2003.

[61] Jan Larsen, L Nonboe, Mads Hintz-Madsen, and Lars Kai Hansen. Design of robust neural network classifiers. In Proceedings of the 1998 IEEE International Conference on Acoustics, Speech and Signal Processing, ICASSP'98 (Cat. No. 98CH36181), volume 2, pages 1205-1208. IEEE, 1998.

[62] Quoc Le and Tomas Mikolov. Distributed representations of sentences and documents. In Proceedings of the 31st International Conference on Machine Learning (ICML-14), pages 1188-1196, 2014.

[63] David D Lewis, Yiming Yang, Tony G Rose, and Fan Li. Rcv1: A new benchmark collection for text categorization research. Journal of machine learning research, 5(Apr):361-397, 2004.

[64] Yunlei Li, Lodewyk FA Wessels, Dick de Ridder, and Marcel JT Reinders. Classification in the presence of class noise using a probabilistic kernel fisher method. Pattern Recognition, 40(12):3349-3357, 2007.

[65] Julián Luengo, Seong-O Shim, Saleh Alshomrani, Abdulrahman Altalhi, and Francisco Herrera. Cnc-nos: Class noise cleaning by ensemble filtering and noise scoring. Knowledge-Based Systems, 140:27-49, 2018.

[66] Andrew L Maas, Raymond E Daly, Peter T Pham, Dan Huang, Andrew Y $\mathrm{Ng}$, and Christopher Potts. Learning word vectors for sentiment analysis. In 
Proceedings of the 49th annual meeting of the association for computational linguistics: Human language technologies-volume 1, pages 142-150. Association for Computational Linguistics, 2011.

[67] Andrea Malossini, Enrico Blanzieri, and Raymond T Ng. Detecting potential labeling errors in microarrays by data perturbation. Bioinformatics, 22(17):21142121, 2006.

[68] Naresh Manwani and PS Sastry. Noise tolerance under risk minimization. IEEE transactions on cybernetics, 43(3):1146-1151, 2013.

[69] Mitchell Marcus, Beatrice Santorini, and Mary Ann Marcinkiewicz. Building a large annotated corpus of english: The penn treebank. 1993.

[70] Oded Maron and Tomás Lozano-Pérez. A framework for multiple-instance learning. In Advances in neural information processing systems, pages 570-576, 1998.

[71] Andrew McCallum, Kamal Nigam, et al. A comparison of event models for naive bayes text classification. In AAAI-98 workshop on learning for text categorization, volume 752, pages 41-48. Citeseer, 1998.

[72] GJ McLachlan. Asymptotic results for discriminant analysis when the initial samples are misclassified. Technometrics, 14(2):415-422, 1972.

[73] Oren Melamud, Jacob Goldberger, and Ido Dagan. context2vec: Learning generic context embedding with bidirectional lstm. In Proceedings of The 20th SIGNLL Conference on Computational Natural Language Learning, pages 5161, 2016.

[74] MR Meybodi and S Lakshmivarahan. Optimality of a general class of learning algorithm. Information science, 28:1-20, 1982.

[75] Tomas Mikolov, Kai Chen, Greg Corrado, and Jeffrey Dean. Efficient estimation of word representations in vector space. arXiv preprint arXiv:1301.3781, 2013. 
[76] Cataldo Musto, Giovanni Semeraro, Marco de Gemmis, and Pasquale Lops. Learning word embeddings from wikipedia for content-based recommender systems. In European Conference on Information Retrieval, pages 729-734. Springer, 2016.

[77] Kumpati Narendra and M.A.L. Thathachar. Learning automata-a survey. IEEE Trans. on Systems, Man, and Cybernetics., SMC-4, 071974.

[78] Kumpati S Narendra and Mandayam AL Thathachar. Learning automata: an introduction. Courier corporation, 2012.

[79] David F Nettleton, Albert Orriols-Puig, and Albert Fornells. A study of the effect of different types of noise on the precision of supervised learning techniques. Artificial intelligence review, 33(4):275-306, 2010.

[80] Curtis G. Northcutt, Lu Jiang, and Isaac L. Chuang. Confident learning: Estimating uncertainty in dataset labels, 2019.

[81] Mohammad S Obaidat, Georgios I. Papadimitriou, Andreas S. Pomportsis, and HS Laskaridis. Learning automata-based bus arbitration for shared-medium atm switches. IEEE Transactions on Systems, Man, and Cybernetics, Part B (Cybernetics), 32(6):815-820, 2002.

[82] Seishi Okamoto and Nobuhiro Yugami. An average-case analysis of the k-nearest neighbor classifier for noisy domains. In IJCAI (1), pages 238-245, 1997.

[83] F. Pedregosa, G. Varoquaux, A. Gramfort, V. Michel, B. Thirion, O. Grisel, M. Blondel, P. Prettenhofer, R. Weiss, V. Dubourg, J. Vanderplas, A. Passos, D. Cournapeau, M. Brucher, M. Perrot, and E. Duchesnay. Scikit-learn: Machine learning in Python. Journal of Machine Learning Research, 12:2825-2830, 2011.

[84] Jeffrey Pennington, Richard Socher, and Christopher D. Manning. Glove: Global vectors for word representation. In Empirical Methods in Natural Language Processing (EMNLP), pages 1532-1543, 2014. 
[85] Matthew E. Peters, Mark Neumann, Mohit Iyyer, Matt Gardner, Christopher Clark, Kenton Lee, and Luke Zettlemoyer. Deep contextualized word representations. CoRR, abs/1802.05365, 2018.

[86] R Pincus. Barnett, v., and lewis t.: Outliers in statistical data. j. wiley \& sons 1994, xvii. 582 pp., 49.95. Biometrical Journal, 37(2):256-256, 1995.

[87] Ronaldo C. Prati, Julián Luengo, and Francisco Herrera. Emerging topics and challenges of learning from noisy data in nonstandard classification: a survey beyond binary class noise. Knowledge and Information Systems, pages 1-35, 2018.

[88] J. Ross Quinlan. Induction of decision trees. Machine learning, 1(1):81-106, 1986.

[89] J. Ross Quinlan. Simplifying decision trees. International journal of manmachine studies, 27(3):221-234, 1987.

[90] Mattias Rantalainen and Chris C Holmes. Accounting for control mislabeling in case-control biomarker studies. Journal of proteome research, 10(12):5562$5567,2011$.

[91] Gunnar Rätsch, Takashi Onoda, and Klaus R Müller. Regularizing adaboost. In Advances in neural information processing systems, pages 564-570, 1999.

[92] Gunnar Rätsch, Takashi Onoda, and Klaus Robert Müller. An improvement of adaboost to avoid overfitting. In Proc. of the Int. Conf. on Neural Information Processing. Citeseer, 1998.

[93] Gunnar Rätsch, Bernhard Schölkopf, Alexander Johannes Smola, Sebastian Mika, Takashi Onoda, and Klaus-Robert Müller. Robust ensemble learning for data mining. In Pacific-Asia Conference on Knowledge Discovery and Data Mining, pages 341-344. Springer, 2000. 
[94] Umaa Rebbapragada and Carla E Brodley. Class noise mitigation through instance weighting. In European Conference on Machine Learning, pages 708715. Springer, 2007.

[95] Marta Recasens, Cristian Danescu-Niculescu-Mizil, and Dan Jurafsky. Linguistic models for analyzing and detecting biased language. In Proceedings of $A C L$, 2013.

[96] K Robbins, S Joseph, W Zhang, R Rekaya, and JK Bertrand. Classification of incipient alzheimer patients using gene expression data: Dealing with potential misdiagnosis. Online Journal of Bioinformatics, 7(9), 2006.

[97] Sebastien Ruder and Julien Eisenschlos. Efficient multi-lingual language model fine-tuning fast.ai nlp, 2019.

[98] M Ruiz, FJ Girón, CJ Pérez, J Martín, and C Rojano. A bayesian model for multinomial sampling with misclassified data. Journal of Applied Statistics, 35(4):369-382, 2008.

[99] José A Sáez, Mikel Galar, Julián Luengo, and Francisco Herrera. Inffc: an iterative class noise filter based on the fusion of classifiers with noise sensitivity control. Information Fusion, 27:19-32, 2016.

[100] José A Sáez, Julián Luengo, Jerzy Stefanowski, and Francisco Herrera. Smoteipf: Addressing the noisy and borderline examples problem in imbalanced classification by a re-sampling method with filtering. Information Sciences, 291:184$203,2015$.

[101] P. S. Sastry, G. D. Nagendra, and Naresh Manwani. A team of continuousaction learning automata for noise-tolerant learning of half-spaces. Trans. Sys. Man Cyber. Part B, 40(1), February 2010.

[102] PS Sastry, GD Nagendra, and Naresh Manwani. A team of continuous-action learning automata for noise-tolerant learning of half-spaces. IEEE Transactions on Systems, Man, and Cybernetics, Part B (Cybernetics), 40(1):19-28, 2009. 
[103] Joseph L Schafer and John W Graham. Missing data: our view of the state of the art. Psychological methods, 7(2):147, 2002.

[104] B Schalkopf and AJ Smola. Learning with kernels. Cambridge: MIT Press. Schakopf, B., Mika, S., Burges, C. J., P. Knirsch, K.-R. M., Retsch, G., Smola, A. J, pages -2000, 112001.

[105] Rico Sennrich, Barry Haddow, and Alexandra Birch. Neural machine translation of rare words with subword units. arXiv preprint arXiv:1508.07909, 2015.

[106] Seyyed Hossein Seyyedi and Behrouz Minaei-Bidgoli. Estimator learning automata for feature subset selection in high-dimensional spaces, case study: Email spam detection. International Journal of Communication Systems, 31(8):e3541, 2018.

[107] Ahmad Abu Shanab, Taghi M Khoshgoftaar, and Randall Wald. Robustness of threshold-based feature rankers with data sampling on noisy and imbalanced data. In Twenty-Fifth International FLAIRS Conference, 2012.

[108] Padhraic Smyth, Usama M Fayyad, Michael C Burl, Pietro Perona, and Pierre Baldi. Inferring ground truth from subjective labelling of venus images. In Advances in neural information processing systems, pages 1085-1092, 1995.

[109] Karen Sparck Jones. A statistical interpretation of term specificity and its application in retrieval. Journal of documentation, 28(1):11-21, 1972.

[110] Tim B Swartz, Yoel Haitovsky, Albert Vexler, and Tae Y Yang. Bayesian identifiability and misclassification in multinomial data. Canadian Journal of Statistics, 32(3):285-302, 2004.

[111] Mahdi Tabassian, Reza Ghaderi, and Reza Ebrahimpour. Knitted fabric defect classification for uncertain labels based on dempster-shafer theory of evidence. Expert Systems with Applications, 38(5):5259-5267, 2011.

[112] Choh-Man Teng. A comparison of noise handling techniques. In FLAIRS Conference, pages 269-273, 2001. 
[113] Mandayam AL Thathachar and Pidaparty S Sastry. Networks of learning automata: Techniques for online stochastic optimization. Springer Science \& Business Media, 2011.

[114] Michael L Tsetlin. On behaviour of finite automata in random medium. Avtom I Telemekhanika, 22(10):1345-1354, 1961.

[115] Jason Van Hulse and Taghi Khoshgoftaar. Knowledge discovery from imbalanced and noisy data. Data \& Knowledge Engineering, 68(12):1513-1542, 2009.

[116] Sofie Verbaeten and Anneleen Van Assche. Ensemble methods for noise elimination in classification problems. In International Workshop on Multiple Classifier Systems, pages 317-325. Springer, 2003.

[117] Armand Vilalta, Dario Garcia-Gasulla, Ferran Parés, Eduard Ayguadé, Jesus Labarta, E Ulises Moya-Sánchez, and Ulises Cortés. Studying the impact of the full-network embedding on multimodal pipelines. Semantic Web, (Preprint):1$15,2018$.

[118] D Randall Wilson and Tony R Martinez. Instance pruning techniques. In $I C M L$, volume 97, pages 400-411, 1997.

[119] D Randall Wilson and Tony R Martinez. Reduction techniques for instancebased learning algorithms. Machine learning, 38(3):257-286, 2000.

[120] Thomas Wolf, Lysandre Debut, Victor Sanh, Julien Chaumond, Clement Delangue, Anthony Moi, Pierric Cistac, Tim Rault, R'emi Louf, Morgan Funtowicz, and Jamie Brew. Huggingface's transformers: State-of-the-art natural language processing. ArXiv, abs/1910.03771, 2019.

[121] Zhilin Yang, Zihang Dai, Yiming Yang, Jaime Carbonell, Ruslan Salakhutdinov, and Quoc V Le. Xlnet: Generalized autoregressive pretraining for language understanding. arXiv preprint arXiv:1906.0823\%, 2019. 
[122] A. Yazidi, X. Zhang, L. Jiao, and B. J. Oommen. The hierarchical continuous pursuit learning automation: A novel scheme for environments with large numbers of actions. IEEE Transactions on Neural Networks and Learning Systems, pages $1-15,2019$.

[123] Chen Zhang, Chunguo Wu, Enrico Blanzieri, You Zhou, Yan Wang, Wei Du, and Yanchun Liang. Methods for labeling error detection in microarrays based on the effect of data perturbation on the regression model. Bioinformatics, 25(20):2708-2714, 2009.

[124] Xingquan Zhu and Xindong Wu. Class noise vs. attribute noise: A quantitative study. Artificial intelligence review, 22(3):177-210, 2004.

[125] Xingquan Zhu, Xindong Wu, and Qijun Chen. Eliminating class noise in large datasets. In Proceedings of the 20th International Conference on Machine Learning (ICML-03), pages 920-927, 2003.

[126] Xingquan Zhu, Xindong Wu, Taghi M Khoshgoftaar, and Yong Shi. An empirical study of the noise impact on cost-sensitive learning. In IJCAI, pages 1168-1174, 2007. 
Appendices 


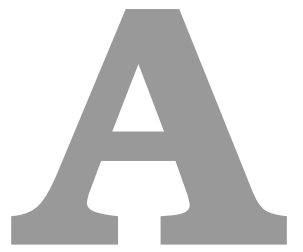

Appendix A 


\begin{tabular}{|l|l|l|l|l|l|l|l|l|}
\hline & AdaBoost & $\begin{array}{l}\text { Decision } \\
\text { Tree }\end{array}$ & kNN & $\begin{array}{l}\text { Naive } \\
\text { Bayes }\end{array}$ & $\begin{array}{l}\text { Logistic } \\
\text { Regression }\end{array}$ & BERT & Tsetlin & Cleanlab \\
\hline \hline $\begin{array}{l}\text { Neighbour- } \\
\text { wise Border }\end{array}$ & 0.72 & 0.60 & 0.61 & 0.75 & 0.75 & 0.73 & 0.80 & 0.74 \\
\hline $\begin{array}{l}\text { Reverse } \\
\text { Non-Linear } \\
\text { Border }\end{array}$ & 0.53 & 0.50 & 0.54 & 0.50 & 0.64 & 0.64 & 0.43 & 0.61 \\
\hline Random & 0.73 & 0.61 & 0.58 & 0.80 & 0.79 & 0.73 & 0.81 & 0.78 \\
\hline $\begin{array}{l}\text { Text } \\
\text { Neighbour- } \\
\text { wise Border }\end{array}$ & 0.73 & 0.61 & 0.61 & 0.74 & 0.75 & 0.73 & 0.80 & 0.74 \\
\hline $\begin{array}{l}\text { Class 1 20\%, } \\
\text { Class 0 10\% }\end{array}$ & 0.54 & 0.51 & 0.55 & 0.50 & 0.79 & 0.69 & 0.80 & 0.76 \\
\hline $\begin{array}{l}\text { Reverse } \\
\text { Neighbour- } \\
\text { wise Border }\end{array}$ & 0.61 & 0.51 & 0.50 & 0.63 & 0.63 & 0.69 & 0.73 & 0.62 \\
\hline Class 1 & 0.51 & 0.50 & 0.52 & 0.50 & 0.78 & 0.63 & 0.77 & 0.69 \\
\hline Length & 0.66 & 0.55 & 0.61 & 0.74 & 0.74 & 0.66 & 0.81 & 0.72 \\
\hline $\begin{array}{l}\text { Class 0 20\%, } \\
\text { Class 1 10\% }\end{array}$ & 0.55 & 0.53 & 0.55 & 0.50 & 0.79 & 0.68 & 0.81 & 0.77 \\
\hline Lexicon & 0.69 & 0.54 & 0.60 & 0.75 & 0.73 & 0.68 & 0.81 & 0.73 \\
\hline $\begin{array}{l}\text { Linear Bor- } \\
\text { der }\end{array}$ & 0.79 & 0.64 & 0.67 & 0.81 & 0.82 & 0.78 & 0.82 & 0.82 \\
\hline $\begin{array}{l}\text { Reverse Text } \\
\text { Neighbour- } \\
\text { wise Border }\end{array}$ & 0.60 & 0.51 & 0.50 & 0.63 & 0.63 & 0.69 & 0.73 & 0.62 \\
\hline Class 0 & 0.51 & 0.51 & 0.53 & 0.49 & 0.78 & 0.62 & 0.80 & 0.72 \\
\hline $\begin{array}{l}\text { Reverse Lin- } \\
\text { ear Border }\end{array}$ & 0.39 & 0.46 & 0.49 & 0.32 & 0.28 & 0.58 & 0.53 & 0.29 \\
\hline $\begin{array}{l}\text { Non-Linear } \\
\text { Border }\end{array}$ & 0.50 & 0.50 & 0.51 & 0.49 & 0.62 & 0.61 & 0.82 & 0.58 \\
\hline None & 0.79 & 0.64 & 0.70 & 0.82 & 0.84 & 0.86 & 0.86 & 0.83 \\
\hline
\end{tabular}

Table A.1: Accuracy obtained from each classifier with $30 \%$ total noise 


\begin{tabular}{|l|l|l|l|l|l|l|l|l|}
\hline & AdaBoost & $\begin{array}{l}\text { Decision } \\
\text { Tree }\end{array}$ & kNN & $\begin{array}{l}\text { Naive } \\
\text { Bayes }\end{array}$ & $\begin{array}{l}\text { Logistic } \\
\text { Regression }\end{array}$ & BERT & Tsetlin & Cleanlab \\
\hline \hline $\begin{array}{l}\text { Neighbour- } \\
\text { wise Border }\end{array}$ & 0.76 & 0.63 & 0.68 & 0.79 & 0.79 & 0.82 & 0.84 & 0.79 \\
\hline $\begin{array}{l}\text { Reverse } \\
\text { Non-Linear } \\
\text { Border }\end{array}$ & 0.66 & 0.53 & 0.61 & 0.75 & 0.78 & 0.71 & 0.58 & 0.71 \\
\hline Random & 0.77 & 0.64 & 0.67 & 0.82 & 0.83 & 0.83 & 0.84 & 0.83 \\
\hline $\begin{array}{l}\text { Text } \\
\text { Neighbour- } \\
\text { wise Border }\end{array}$ & 0.76 & 0.63 & 0.65 & 0.78 & 0.78 & 0.79 & 0.83 & 0.79 \\
\hline $\begin{array}{l}\text { Class 1 10\%, } \\
\text { Class 0 5\% }\end{array}$ & 0.74 & 0.64 & 0.65 & 0.63 & 0.83 & 0.75 & 0.83 & 0.80 \\
\hline $\begin{array}{l}\text { Reverse } \\
\text { Neighbour- } \\
\text { wise Border }\end{array}$ & 0.70 & 0.58 & 0.60 & 0.73 & 0.74 & 0.75 & 0.80 & 0.72 \\
\hline Class 1 & 0.66 & 0.57 & 0.61 & 0.67 & 0.81 & 0.75 & 0.82 & 0.76 \\
\hline Length & 0.73 & 0.60 & 0.66 & 0.78 & 0.79 & 0.76 & 0.84 & 0.77 \\
\hline $\begin{array}{l}\text { Class 0 10\%, } \\
\text { Class 1 5\% }\end{array}$ & 0.67 & 0.58 & 0.63 & 0.66 & 0.83 & 0.75 & 0.84 & 0.80 \\
\hline Lexicon & 0.74 & 0.60 & 0.66 & 0.79 & 0.79 & 0.78 & 0.84 & 0.79 \\
\hline $\begin{array}{l}\text { Linear Bor- } \\
\text { der }\end{array}$ & 0.79 & 0.64 & 0.70 & 0.81 & 0.83 & 0.82 & 0.84 & 0.83 \\
\hline $\begin{array}{l}\text { Reverse Text } \\
\text { Neighbour- } \\
\text { wise Border }\end{array}$ & 0.71 & 0.58 & 0.60 & 0.73 & 0.74 & 0.78 & 0.80 & 0.73 \\
\hline Class 0 & 0.66 & 0.58 & 0.63 & 0.65 & 0.82 & 0.75 & 0.85 & 0.79 \\
\hline $\begin{array}{l}\text { Reverse Lin- } \\
\text { ear Border }\end{array}$ & 0.51 & 0.50 & 0.55 & 0.45 & 0.40 & 0.69 & 0.65 & 0.42 \\
\hline $\begin{array}{l}\text { Non-Linear } \\
\text { Border }\end{array}$ & 0.64 & 0.57 & 0.61 & 0.68 & 0.73 & 0.71 & 0.85 & 0.68 \\
\hline
\end{tabular}

Table A.2: Accuracy obtained from each classifier with $15 \%$ total noise 


\begin{tabular}{|c|c|c|c|}
\hline Accuracy \% & S & Number of States & Number of Clauses \\
\hline \hline 60.41 & 3.0 & 16.0 & 4.0 \\
\hline 99.94 & 3.0 & 256.0 & 4.0 \\
\hline 99.72 & 3.0 & 65536.0 & 4.0 \\
\hline 70.74 & 3.0 & 16.0 & 10.0 \\
\hline 99.92 & 3.0 & 256.0 & 10.0 \\
\hline 99.74 & 3.0 & 65536.0 & 10.0 \\
\hline 88.96 & 3.0 & 16.0 & 100.0 \\
\hline 99.90 & 3.0 & 256.0 & 100.0 \\
\hline 99.71 & 3.0 & 65536.0 & 100.0 \\
\hline 59.74 & 10.0 & 16.0 & 4.0 \\
\hline 74.75 & 10.0 & 256.0 & 4.0 \\
\hline 74.03 & 10.0 & 65536.0 & 4.0 \\
\hline 68.25 & 10.0 & 16.0 & 10.0 \\
\hline 81.77 & 10.0 & 256.0 & 10.0 \\
\hline 82.51 & 10.0 & 65536.0 & 10.0 \\
\hline 92.47 & 10.0 & 16.0 & 100.0 \\
\hline 95.89 & 10.0 & 256.0 & 100.0 \\
\hline 95.86 & 10.0 & 65536.0 & 100.0 \\
\hline 52.54 & 100.0 & 16.0 & 4.0 \\
\hline 52.85 & 100.0 & 256.0 & 4.0 \\
\hline 52.94 & 100.0 & 65536.0 & 4.0 \\
\hline 54.77 & 100.0 & 16.0 & 10.0 \\
\hline 55.12 & 100.0 & 256.0 & 10.0 \\
\hline 54.96 & 100.0 & 65536.0 & 10.0 \\
\hline 72.91 & 100.0 & 16.0 & 100.0 \\
\hline 72.78 & 100.0 & 256.0 & 100.0 \\
\hline 73.00 & 100.0 & 65536.0 & 100.0 \\
\hline & & & \\
\hline & & & \\
\hline & & & \\
\hline & & & 0 \\
\hline
\end{tabular}

Table A.3: Accuracy of the XOR dataset with different parameter settings, injected with $15 \%$ noise 


\begin{tabular}{|c|c|c|c|}
\hline Accuracy \% & S & Number of States & Number of Clauses \\
\hline \hline 51.28 & 3.0 & 16.0 & 4.0 \\
\hline 97.01 & 3.0 & 256.0 & 4.0 \\
\hline 95.22 & 3.0 & 65536.0 & 4.0 \\
\hline 52.63 & 3.0 & 16.0 & 10.0 \\
\hline 96.48 & 3.0 & 256.0 & 10.0 \\
\hline 96.43 & 3.0 & 65536.0 & 10.0 \\
\hline 55.76 & 3.0 & 16.0 & 100.0 \\
\hline 97.67 & 3.0 & 256.0 & 100.0 \\
\hline 96.82 & 3.0 & 65536.0 & 100.0 \\
\hline 51.93 & 10.0 & 16.0 & 4.0 \\
\hline 71.24 & 10.0 & 256.0 & 4.0 \\
\hline 70.78 & 10.0 & 65536.0 & 4.0 \\
\hline 54.85 & 10.0 & 16.0 & 10.0 \\
\hline 79.32 & 10.0 & 256.0 & 10.0 \\
\hline 80.21 & 10.0 & 65536.0 & 10.0 \\
\hline 64.83 & 10.0 & 16.0 & 100.0 \\
\hline 91.06 & 10.0 & 256.0 & 100.0 \\
\hline 90.58 & 10.0 & 65536.0 & 100.0 \\
\hline 51.58 & 100.0 & 16.0 & 4.0 \\
\hline 52.29 & 100.0 & 256.0 & 4.0 \\
\hline 52.37 & 100.0 & 65536.0 & 4.0 \\
\hline 52.96 & 100.0 & 16.0 & 10.0 \\
\hline 53.76 & 100.0 & 256.0 & 10.0 \\
\hline 54.02 & 100.0 & 65536.0 & 10.0 \\
\hline 64.40 & 100.0 & 16.0 & 100.0 \\
\hline 66.42 & 100.0 & 256.0 & 100.0 \\
\hline 66.61 & 100.0 & 65536.0 & 100.0 \\
\hline & & & \\
\hline & & & \\
\hline & & & \\
\hline & & & 0 \\
\hline
\end{tabular}

Table A.4: Accuracy of the XOR dataset with different parameter settings, injected with $30 \%$ noise 